EUROPEAN ORGANIZATION FOR NUCLEAR RESEARCH

CERN-PH-EP/2004-020

OPAL PR 399

26th March 2004

Revised 13 May 2004

\title{
Search for Neutral Higgs Bosons in CP-Conserving and CP-Violating MSSM Scenarios
}

\author{
The OPAL Collaboration
}

\begin{abstract}
This report summarizes the final results from the OPAL collaboration on searches for neutral Higgs bosons predicted by the Minimal Supersymmetric Standard Model (MSSM). CP-conserving and, for the first time at LEP, CP-violating scenarios are studied. New scenarios are also included, which aim to set the stage for Higgs searches at future colliders. The results are based on the data collected with the OPAL detector at $\mathrm{e}^{+} \mathrm{e}^{-}$centre-ofmass energies up to $209 \mathrm{GeV}$. The data are consistent with the prediction of the Standard Model with no Higgs boson produced. Model-independent limits are derived for the cross-sections of a number of event topologies motivated by predictions of the MSSM. Limits on Higgs boson masses and other MSSM parameters are obtained for a number of representative MSSM benchmark scenarios. For example, in the CP-conserving scenario $m_{\mathrm{h}}-\max$ where the MSSM parameters are adjusted to predict the largest range of values for $m_{\mathrm{h}}$ at each $\tan \beta$, and for a top quark mass of $174.3 \mathrm{GeV}$, the domain $0.7<\tan \beta<1.9$ is excluded at the $95 \%$ confidence level and Higgs boson mass limits of $m_{\mathrm{h}}>84.5 \mathrm{GeV}$ and $m_{\mathrm{A}}>85.0 \mathrm{GeV}$ are obtained. For the CP-violating benchmark scenario CPX which, by construction, enhances the CP-violating effects in the Higgs sector, the domain $\tan \beta<2.8$ is excluded but no universal limit can be set on the Higgs boson masses.
\end{abstract}

( To be submitted to European Physical Journal $\boldsymbol{C}$ ) 


\section{The OPAL Collaboration}

G. Abbiendi ${ }^{2}$, C. Ainsley ${ }^{5}$, P.F. Åkesson ${ }^{3, y}$, G. Alexander ${ }^{22}$, J. Allison ${ }^{16}$, P. Amaral $^{9}$, G. Anagnostou ${ }^{1}$, K.J. Anderson ${ }^{9}$, S. Asai ${ }^{23}$, D. Axen ${ }^{27}$, G. Azuelos ${ }^{18, a}$, I. Bailey ${ }^{26}$,

E. Barberio $^{8, p}$, T. Barillari ${ }^{32}$, R.J. Barlow ${ }^{16}$, R.J. Batley ${ }^{5}$, P. Bechtle ${ }^{25}$, T. Behnke ${ }^{25}$, K.W. Bell ${ }^{20}$, P.J. Bell ${ }^{1}$, G. Bella ${ }^{22}$, A. Bellerive ${ }^{6}$, G. Benelli ${ }^{4}$, S. Bethke ${ }^{32}$, O. Biebel ${ }^{31}$, O. Boeriu ${ }^{10}$, P. Bock ${ }^{11}$, M. Boutemeur ${ }^{31}$, S. Braibant ${ }^{8}$, L. Brigliadori ${ }^{2}$, R.M. Brown ${ }^{20}$, K. Buesser ${ }^{25}$, H.J. Burckhart ${ }^{8}$, S. Campana ${ }^{4}$, R.K. Carnegie ${ }^{6}$, A.A. Carter $^{13}$, J.R. Carter ${ }^{5}$, C.Y. Chang ${ }^{17}$, D.G. Charlton ${ }^{1}$, C. Ciocca ${ }^{2}$, A. Csilling ${ }^{29}$, M. Cuffiani ${ }^{2}$, S. Dado ${ }^{21}$, A. De Roeck $^{8}$, E.A. De Wolf ${ }^{8, s}$, K. Desch ${ }^{25}$, B. Dienes ${ }^{30}$, M. Donkers ${ }^{6}$, J. Dubbert ${ }^{31}$,

E. Duchovni ${ }^{24}$, G. Duckeck ${ }^{31}$, I.P. Duerdoth ${ }^{16}$, E. Etzion ${ }^{22}$, F. Fabbri ${ }^{2}$, L. Feld ${ }^{10}$, P. Ferrari ${ }^{8}$, F. Fiedler ${ }^{31}$, I. Fleck ${ }^{10}$, M. Ford ${ }^{5}$, A. Frey ${ }^{8}$, P. Gagnon ${ }^{12}$, J.W. Gary ${ }^{4}$,

G. Gaycken ${ }^{25}$, C. Geich-Gimbel ${ }^{3}$, G. Giacomelli², P. Giacomelli² , M. Giunta ${ }^{4}$,

J. Goldberg ${ }^{21}$, E. Gross ${ }^{24}$, J. Grunhaus ${ }^{22}$, M. Gruwé ${ }^{8}$, P.O. Günther ${ }^{3}$, A. Gupta ${ }^{9}$, C.Hajdu ${ }^{29}$, M.Hamann ${ }^{25}$, G.G.Hanson ${ }^{4}$, A. Harel ${ }^{21}$, M.Hauschild ${ }^{8}$, C.M.Hawkes ${ }^{1}$, R. Hawkings ${ }^{8}$, R.J. Hemingway ${ }^{6}$, G. Herten ${ }^{10}$, R.D. Heuer ${ }^{25}$, J.C. Hill ${ }^{5}$, K. Hoffman ${ }^{9}$, D. Horváth ${ }^{29, c}$, P. Igo-Kemenes ${ }^{11}$, K. Ishii ${ }^{23}$, H. Jeremie ${ }^{18}$, P. Jovanovic ${ }^{1}$, T.R. Junk ${ }^{6, i}$, N. Kanaya ${ }^{26}$, J. Kanzaki ${ }^{23, u}$, D. Karlen ${ }^{26}$, K. Kawagoe ${ }^{23}$, T. Kawamoto ${ }^{23}$, R.K. Keeler ${ }^{26}$, R.G. Kellogg ${ }^{17}$, B.W. Kennedy ${ }^{20}$, S. Kluth ${ }^{32}$, T. Kobayashi ${ }^{23}$, M. Kobel ${ }^{3}$, S. Komamiya ${ }^{23}$,

T. Krämer ${ }^{25}$, P. Krieger ${ }^{6, l}$, J. von Krogh ${ }^{11}$, K. Kruger ${ }^{8}$, T. Kuhl ${ }^{25}$, M. Kupper ${ }^{24}$, G.D. Lafferty ${ }^{16}$, H. Landsman ${ }^{21}$, D. Lanske ${ }^{14}$, J.G. Layter ${ }^{4}$, D. Lellouch ${ }^{24}$, J. Letts ${ }^{o}$, L. Levinson ${ }^{24}$, J. Lillich ${ }^{10}$, S.L. Lloyd ${ }^{13}$, F.K. Loebinger ${ }^{16}$, J. Lu ${ }^{27, w}$, A. Ludwig ${ }^{3}$, J. Ludwig ${ }^{10}$, W. Mader ${ }^{3}$, S. Marcellini ${ }^{2}$, A.J. Martin ${ }^{13}$, G. Masetti ${ }^{2}$, T. Mashimo ${ }^{23}$, P. Mättig ${ }^{m}$, J. McKenna ${ }^{27}$, R.A. McPherson ${ }^{26}$, F. Meijers ${ }^{8}$, W. Menges ${ }^{25}$, F.S. Merritt ${ }^{9}$, H. Mes ${ }^{6, a}$, N. Meyer ${ }^{25}$, A. Michelini ${ }^{2}$, S. Mihara ${ }^{23}$, G. Mikenberg ${ }^{24}$, D.J. Miller ${ }^{15}$, S. Moed ${ }^{21}$, W. Mohr ${ }^{10}$, T. Mori ${ }^{23}$, A. Mutter ${ }^{10}$, K. Nagai ${ }^{13}$, I. Nakamura ${ }^{23, v}$, H. Nanjo ${ }^{23}$,

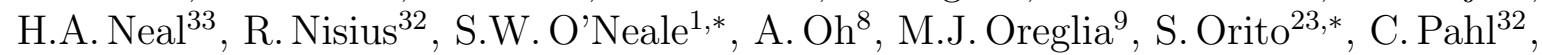
G. Pásztor ${ }^{4, g}$, J.R. Pater ${ }^{16}$, J.E. Pilcher ${ }^{9}$, J. Pinfold ${ }^{28}$, D.E. Plane ${ }^{8}$, B. Poli ${ }^{2}$, O. Pooth ${ }^{14}$, M. Przybycień ${ }^{8, n}$, A. Quadt ${ }^{3}$, K. Rabbertz ${ }^{8, r}$, C. Rembser $^{8}$, P. Renkel ${ }^{24}$, J.M. Roney ${ }^{26}$,

Y.Rozen ${ }^{21}$, K. Runge ${ }^{10}$, K. Sachs ${ }^{6}$, T. Saeki ${ }^{23}$, E.K.G.Sarkisyan ${ }^{8, j}$, A.D. Schaile ${ }^{31}$, O. Schaile ${ }^{31}$, P. Scharff-Hansen ${ }^{8}$, J. Schieck ${ }^{32}$, T.Schörner-Sadenius ${ }^{8, z}$, M. Schröder ${ }^{8}$, M.Schumacher ${ }^{3}$, W.G.Scott ${ }^{20}$, R. Seuster ${ }^{14, f}$, T.G.Shears ${ }^{8, h}$, B.C. Shen ${ }^{4}$, P. Sherwood ${ }^{15}$, A.Skuja ${ }^{17}$, A.M.Smith ${ }^{8}$, R. Sobie ${ }^{26}$, S.Söldner-Rembold ${ }^{15}$, F.Spano ${ }^{9}$, A.Stahl ${ }^{3, x}$, D. Strom ${ }^{19}$, R. Ströhmer ${ }^{31}$, S. Tarem ${ }^{21}$, M. Tasevsky ${ }^{8, s}$, R. Teuscher ${ }^{9}$, M.A. Thomson ${ }^{5}$,

E. Torrence ${ }^{19}$, D. Toya ${ }^{23}$, P. $\operatorname{Tran}^{4}$, I. Trigger ${ }^{8}$, Z. Trócsányi ${ }^{30, e}$, E. Tsur $^{22}$,

M.F. Turner-Watson ${ }^{1}$, I. Ueda ${ }^{23}$, B. Ujvári ${ }^{30, e}$, C.F. Vollmer ${ }^{31}$, P. Vannerem ${ }^{10}$, R. Vértesi ${ }^{30, e}$, M. Verzocchi ${ }^{17}$, H. Voss ${ }^{8, q}$, J. Vossebeld ${ }^{8, h}$, C.P. Ward ${ }^{5}$, D.R. Ward ${ }^{5}$, P.M. Watkins ${ }^{1}$, A.T. Watson ${ }^{1}$, N.K. Watson ${ }^{1}$, P.S. Wells ${ }^{8}$, T. Wengler ${ }^{8}$, N. Wermes ${ }^{3}$, G.W.Wilson ${ }^{16, k}$, J.A. Wilson ${ }^{1}$, G. Wolf ${ }^{24}$, T.R. Wyatt ${ }^{16}$, S. Yamashita ${ }^{23}$, D. Zer-Zion ${ }^{4}$, L. Zivkovic ${ }^{24}$

${ }^{1}$ School of Physics and Astronomy, University of Birmingham, Birmingham B15 2TT, UK ${ }^{2}$ Dipartimento di Fisica dell' Università di Bologna and INFN, I-40126 Bologna, Italy

${ }^{3}$ Physikalisches Institut, Universität Bonn, D-53115 Bonn, Germany

${ }^{4}$ Department of Physics, University of California, Riverside CA 92521, USA

${ }^{5}$ Cavendish Laboratory, Cambridge CB3 0HE, UK

${ }^{6}$ Ottawa-Carleton Institute for Physics, Department of Physics, Carleton University, Ottawa, Ontario K1S 5B6, Canada 
${ }^{8}$ CERN, European Organisation for Nuclear Research, CH-1211 Geneva 23, Switzerland

${ }^{9}$ Enrico Fermi Institute and Department of Physics, University of Chicago, Chicago IL 60637, USA

${ }^{10}$ Fakultät für Physik, Albert-Ludwigs-Universität Freiburg, D-79104 Freiburg, Germany

${ }^{11}$ Physikalisches Institut, Universität Heidelberg, D-69120 Heidelberg, Germany

${ }^{12}$ Indiana University, Department of Physics, Bloomington IN 47405, USA

${ }^{13}$ Queen Mary and Westfield College, University of London, London E1 4NS, UK

${ }^{14}$ Technische Hochschule Aachen, III Physikalisches Institut, Sommerfeldstrasse 26-28, D52056 Aachen, Germany

${ }^{15}$ University College London, London WC1E 6BT, UK

${ }^{16}$ Department of Physics, Schuster Laboratory, The University, Manchester M13 9PL, UK

${ }^{17}$ Department of Physics, University of Maryland, College Park, MD 20742, USA

${ }^{18}$ Laboratoire de Physique Nucléaire, Université de Montréal, Montréal, Québec H3C 3J7, Canada

${ }^{19}$ University of Oregon, Department of Physics, Eugene OR 97403, USA

${ }^{20}$ CCLRC Rutherford Appleton Laboratory, Chilton, Didcot, Oxfordshire OX11 0QX, UK

${ }^{21}$ Department of Physics, Technion-Israel Institute of Technology, Haifa 32000, Israel

${ }^{22}$ Department of Physics and Astronomy, Tel Aviv University, Tel Aviv 69978, Israel

${ }^{23}$ International Centre for Elementary Particle Physics and Department of Physics, University of Tokyo, Tokyo 113-0033, and Kobe University, Kobe 657-8501, Japan

${ }^{24}$ Particle Physics Department, Weizmann Institute of Science, Rehovot 76100, Israel

${ }^{25}$ Universität Hamburg/DESY, Institut für Experimentalphysik, Notkestrasse 85, D-22607 Hamburg, Germany

${ }^{26}$ University of Victoria, Department of Physics, P O Box 3055, Victoria BC V8W 3P6, Canada

${ }^{27}$ University of British Columbia, Department of Physics, Vancouver BC V6T 1Z1, Canada

${ }^{28}$ University of Alberta, Department of Physics, Edmonton AB T6G 2J1, Canada

${ }^{29}$ Research Institute for Particle and Nuclear Physics, H-1525 Budapest, P O Box 49, Hungary

${ }^{30}$ Institute of Nuclear Research, H-4001 Debrecen, P O Box 51, Hungary

${ }^{31}$ Ludwig-Maximilians-Universität München, Sektion Physik, Am Coulombwall 1, D85748 Garching, Germany

${ }^{32}$ Max-Planck-Institute für Physik, Föhringer Ring 6, D-80805 München, Germany

${ }^{33}$ Yale University, Department of Physics, New Haven, CT 06520, USA

${ }^{a}$ and at TRIUMF, Vancouver, Canada V6T $2 \mathrm{~A} 3$

${ }^{c}$ and Institute of Nuclear Research, Debrecen, Hungary

${ }^{e}$ and Department of Experimental Physics, University of Debrecen, Hungary

$f$ and MPI München

$g$ and Research Institute for Particle and Nuclear Physics, Budapest, Hungary

${ }^{h}$ now at University of Liverpool, Dept of Physics, Liverpool L69 3BX, U.K.

${ }^{i}$ now at Dept. Physics, University of Illinois at Urbana-Champaign, U.S.A.

$j$ and Manchester University

${ }^{k}$ now at University of Kansas, Dept of Physics and Astronomy, Lawrence, KS 66045, U.S.A.

${ }^{l}$ now at University of Toronto, Dept of Physics, Toronto, Canada 
${ }^{m}$ current address Bergische Universität, Wuppertal, Germany

${ }^{n}$ now at University of Mining and Metallurgy, Cracow, Poland

${ }^{o}$ now at University of California, San Diego, U.S.A.

${ }^{p}$ now at The University of Melbourne, Victoria, Australia

${ }^{q}$ now at IPHE Université de Lausanne, CH-1015 Lausanne, Switzerland

${ }^{r}$ now at IEKP Universität Karlsruhe, Germany

${ }^{s}$ now at University of Antwerpen, Physics Department,B-2610 Antwerpen, Belgium; supported by Interuniversity Attraction Poles Programme - Belgian Science Policy

$u$ and High Energy Accelerator Research Organisation (KEK), Tsukuba, Ibaraki, Japan

${ }^{v}$ now at University of Pennsylvania, Philadelphia, Pennsylvania, USA

${ }^{w}$ now at TRIUMF, Vancouver, Canada

${ }^{x}$ now at DESY Zeuthen

$y$ now at CERN

${ }^{z}$ now at DESY

* Deceased 


\section{Introduction}

It is generally assumed that the Higgs mechanism [1] is responsible for the breaking of electroweak symmetry and for the generation of elementary particle masses. In its simplest form, implemented in the Standard Model (SM), this mechanism implies the self-interactions of one doublet of complex scalar Higgs fields and predicts the existence of one physical scalar particle, the SM Higgs boson. The mass of this particle is not predicted by the model. Despite an intense effort over the last few decades, the SM Higgs boson has not been detected; a lower bound of $114.4 \mathrm{GeV}$ has been set on its mass [2].

The SM, while successfully describing all electroweak phenomena investigated so far, provides no mechanism for stabilizing the electroweak energy scale $\left(\approx M_{\mathrm{W}}\right)$ in the presence of quantum corrections (the "scale hierarchy" problem). Supersymmetry (SUSY) is proposed as a possible solution to this problem since in SUSY models fermionic quantum corrections are compensated by bosonic corrections of similar size, and vice versa.

The Minimal Supersymmetric Standard Model (MSSM) is the SUSY extension of the SM with minimal new particle content. It introduces two complex Higgs field doublets which is the minimal Higgs structure required to keep the theory free of anomalies and to give masses to all fermions. The MSSM predicts five Higgs bosons: three neutral and two charged particles. At least one of the neutral Higgs bosons is predicted to have its mass close to the electroweak energy scale; when radiative corrections are included, this mass should be less than $135 \mathrm{GeV}$ 3. This prediction provides a strong motivation for searches at present and future colliders.

In the MSSM the Higgs potential is invariant under CP transformation at tree level. However, it is possible to break CP symmetry in the Higgs sector by radiative corrections, especially by contributions from third generation scalar-quarks [4. Such a scenario is theoretically attractive since it provides a possible solution to the cosmic baryon asymmetry [5] while the size of the CP-violating (CPV) effects occurring in the SM are far too small to account for it.

The searches performed so far at LEP have been restricted to CP-conserving (CPC) MSSM scenarios where all CPV phases in the soft SUSY-breaking Lagrangian related to the Higgs sector are put to zero. Under this assumption the three neutral Higgs bosons are $\mathrm{CP}$ eigenstates: the $\mathrm{h}$ and $\mathrm{H}$ bosons ( $\mathrm{h}$ is defined to be the lighter of the two) are $\mathrm{CP}$-even and the $\mathrm{A}$ boson is $\mathrm{CP}$-odd. Of these, only the $\mathrm{CP}$-even states couple to the $\mathrm{Z}$ boson in Higgsstrahlung at tree level; thus, at LEP energies, these particles are mainly produced through the Higgsstrahlung processes $\mathrm{e}^{+} \mathrm{e}^{-} \rightarrow \mathrm{hZ}$ and $\mathrm{HZ}$, and the pair production processes $\mathrm{e}^{+} \mathrm{e}^{-} \rightarrow \mathrm{hA}$ and $\mathrm{HA}\left(\mathrm{e}^{+} \mathrm{e}^{-} \rightarrow \mathrm{hH}\right.$ is forbidden by angular momentum and $\mathrm{CP}$ conservation). Production of $\mathrm{h}$ and $\mathrm{H}$ via $\mathrm{WW}$ and $\mathrm{ZZ}$ fusion in the $t$-channel, where a Higgs particle is produced in association with a pair of neutrinos or electrons, is included and plays a role at the kinematic limit of Higgs boson production at LEP energies.

In this paper CPV MSSM scenarios are also considered. In such scenarios the three neutral Higgs bosons, $\mathrm{H}_{i}(i=1,2,3)$ are mixtures of the CP-even and CP-odd Higgs fields. Consequently, they all couple to the $\mathrm{Z}$ boson by their $\mathrm{CP}$-even field components and to each other. Therefore, the Higgsstrahlung processes $\mathrm{e}^{+} \mathrm{e}^{-} \rightarrow \mathrm{H}_{i} \mathrm{Z}(i=1,2,3)$ and pair production processes $\mathrm{e}^{+} \mathrm{e}^{-} \rightarrow \mathrm{H}_{i} \mathrm{H}_{j}(i \neq j)$ may all occur, however with widely varying cross-sections. In large domains of the model parameters, the lightest neutral Higgs boson $\mathrm{H}_{1}$ may escape detection, since its coupling to the $\mathrm{Z}$ boson may be too weak. The other two Higgs boson masses may be out of reach on kinematic grounds or may also have small 
production cross-sections. As a result, the exclusion limits obtained previously for the CPC scenario may be invalidated by CPV effects.

The decay properties of the Higgs bosons, while being quantitatively different in the two scenarios, maintain a certain similarity. Since Higgs bosons, in general, couple to mass, the largest branching ratios are those to $\mathrm{b} \overline{\mathrm{b}}$ and $\tau^{+} \tau^{-}$pairs. If kinematically allowed, the cascade decays $\mathrm{h} \rightarrow \mathrm{AA}$ (CPC scenario) 6] or $\mathrm{H}_{2} \rightarrow \mathrm{H}_{1} \mathrm{H}_{1}$ (CPV scenario) would occur and can even be the dominant decays. The searches described below take this possibility into account.

Since the topological searches are essentially insensitive to the CP parity of the Higgs bosons, their results can be applied both to the CPC and CPV MSSM scenarios. For example, the search for the Higgsstrahlung signal topology $\mathrm{e}^{+} \mathrm{e}^{-} \rightarrow \mathrm{hZ}$ in CPC scenarios can be interpreted as a search for the processes $\mathrm{e}^{+} \mathrm{e}^{-} \rightarrow \mathrm{H}_{1} \mathrm{Z}$ and $\mathrm{H}_{2} \mathrm{Z}$ in CPV scenarios; similarly, the search for the pair production process $\mathrm{e}^{+} \mathrm{e}^{-} \rightarrow \mathrm{hA}$ in $\mathrm{CPC}$ scenarios can be interpreted as a search for the process $\mathrm{e}^{+} \mathrm{e}^{-} \rightarrow \mathrm{H}_{1} \mathrm{H}_{2}$ in $\mathrm{CPV}$ scenarios.

The results presented in this paper are based on all data collected by the OPAL collaboration, including LEP1 data taken at the $\mathrm{Z}$ resonance and LEP2 data collected between 130 and $209 \mathrm{GeV}$. Many of the searches are already described in earlier OPAL publications [7, 8, 9, 10, 11, 12, 13. In this paper we describe only the optimizations of the searches for the data collected at the highest energies, between $\sqrt{s}=192$ and $209 \mathrm{GeV}$. The old and new searches are combined to produce the final results presented here.

Recent searches for neutral Higgs bosons performed by other LEP collaborations, limited to the CPC MSSM scenarios, are listed in 14 while results from the Tevatron collaborations are reported in [15].

This paper is organised as follows. Section 2 summarises the main features of the OPAL detector and the Monte Carlo simulations. Section 3 describes the model predictions for Higgs boson production. The search topologies and the experimental searches for the corresponding final states are described in Section 4, with emphasis on those searches which have not been covered in earlier OPAL publications. In Section 5 the statistical combination of individual search channels and the derivation of exclusion limits in the MSSM parameter space are described. In Section [6 the search results are translated into model-independent limits on topological cross-sections. In Section 7 the MSSM benchmark scenarios, including those recently suggested in [16], and the corresponding results are presented. A short summary is given in Section 8 .

\section{OPAL detector and Monte Carlo samples}

The OPAL detector is described in [17]. The tracking detectors and calorimeters have nearly complete solid angle coverage. The central tracking detector is placed in a uniform axial magnetic field of $0.435 \mathrm{~T}$. The innermost part is occupied by a high-resolution silicon strip ("microvertex") detector [18. It surrounds the beam pipe and covers the polar angle ${ }^{1}$ range $|\cos \theta|<0.93$, and is the basic tool for an efficient b-tagging [9]. This detector is followed by a high-precision vertex drift chamber, a large-volume jet chamber, and chambers to measure the $z$ coordinates along the particle trajectories. A lead-glass

\footnotetext{
${ }^{1}$ OPAL uses a right-handed coordinate system where the $+z$ direction is along the electron beam and where $+x$ points to the centre of the LEP ring. The polar angle $\theta$ is defined with respect to the $+z$ direction and the azimuthal angle $\phi$ with respect to the $+x$ direction. The centre of the $\mathrm{e}^{+} \mathrm{e}^{-} \operatorname{collision}$ region defines the origin of the coordinate system.
} 
electromagnetic calorimeter with a presampler is located outside the magnet coil. The iron return-yoke of the magnet is instrumented with streamer tubes and thin-gap chambers for hadron calorimetry. Finally, the detector is completed by several layers of muon chambers.

A variety of Monte Carlo samples are generated to estimate the detection efficiencies for the Higgs boson signal and to optimise the rejection of the background. The production cross-section and kinematic properties of the Higgs boson signal vary rapidly with energy near the kinematic limit. For an accurate modelling, the signal and background samples are generated at several centre-of-mass energies, from $192 \mathrm{GeV}$ to $210 \mathrm{GeV}$. The main background processes are generated with 15 to 25 times the statistics of the data.

Signal events for the Higgsstrahlung and pair production search channels are generated using the HZHA03 [19] program. The pair production samples are described in this paper. For Higgsstrahlung, the Monte Carlo samples used are described in [7. Typical sample sizes are 2000 to 5000 signal events per Higgs mass point. For the background processes the following event generators are used: KK2f [20] for $(\mathrm{Z} / \gamma)^{*} \rightarrow \mathrm{q} \overline{\mathrm{q}}(\gamma), \mu^{+} \mu^{-}(\gamma)$ and $\tau^{+} \tau^{-}(\gamma)$, grc4f [21] for four-fermion processes (4f), BHWIDE [22] for $\mathrm{e}^{+} \mathrm{e}^{-}(\gamma)$, and PHOJET [23], HERWIG 24], and Vermaseren [25] for hadronic and leptonic two-photon processes $(\gamma \gamma)$. JETSET [26] is used as the principal model for fragmentation and hadronisation. The detector response is simulated in full detail [27].

\section{$3 \quad$ Higgs boson production processes}

In the MSSM, the Higgsstrahlung and pair production processes have complementary cross-sections. Their relative rate is regulated by sum rules which are different in the $\mathrm{CPC}$ and CPV scenarios and depend on the precise choices of the MSSM parameters.

In the CPC scenario, the cross-sections for the processes $\mathrm{e}^{+} \mathrm{e}^{-} \rightarrow \mathrm{hZ}, \mathrm{e}^{+} \mathrm{e}^{-} \rightarrow \mathrm{HZ}$ and $\mathrm{e}^{+} \mathrm{e}^{-} \rightarrow \mathrm{hA}$ are given by

$$
\begin{aligned}
\mathrm{e}^{+} \mathrm{e}^{-} \rightarrow \mathrm{hZ}: & \sigma_{\mathrm{hZ}}=\sin ^{2}(\beta-\alpha) \sigma_{\mathrm{HZ}}^{\mathrm{SM}}\left(m_{\mathrm{h}}\right), \\
\mathrm{e}^{+} \mathrm{e}^{-} \rightarrow \mathrm{HZ}: & \sigma_{\mathrm{HZ}}=\cos ^{2}(\beta-\alpha) \sigma_{\mathrm{HZ}}^{\mathrm{SM}}\left(m_{\mathrm{H}}\right), \\
\mathrm{e}^{+} \mathrm{e}^{-} \rightarrow \mathrm{hA}: & \sigma_{\mathrm{hA}}=\cos ^{2}(\beta-\alpha) \bar{\lambda} \sigma_{\mathrm{HZ}}^{\mathrm{SM}}\left(m_{\mathrm{h}}\right),
\end{aligned}
$$

where $\sigma_{\mathrm{HZ}}^{\mathrm{SM}}$ is the cross-section for the SM Higgsstrahlung process $\mathrm{e}^{+} \mathrm{e}^{-} \rightarrow \mathrm{H}_{\mathrm{SM}} \mathrm{Z}, \tan \beta=$ $v_{2} / v_{1}$ is the ratio of the vacuum expectation values of the two Higgs field doublets coupling to "up" $\left(v_{2}\right)$ and "down" $\left(v_{1}\right)$ type fermions, and $\alpha$ is the mixing angle describing the combination of the two CP-even weak eigenstates to produce the two CP-even Higgs mass eigenstates. The symbol $\bar{\lambda}$ denotes the kinematic phase-space factor

$$
\bar{\lambda}=\frac{\lambda_{\mathrm{Ah}}^{3 / 2}}{\lambda_{\mathrm{Zh}}^{1 / 2}\left(12 M_{\mathrm{Z}}^{2} / s+\lambda_{\mathrm{Zh}}\right)}
$$

with

$$
\lambda_{i j}=\frac{1-\left(m_{i}+m_{j}\right)^{2} / s}{1-\left(m_{i}-m_{j}\right)^{2} / s} .
$$

Due to the complementarity of the Higgsstrahlung and pair production processes, expressed in Equations (11) and (3), the searches have to include both of them in order to maintain a high sensitivity over the whole MSSM parameter space. 
Similar, but more complex, sum rules regulate the relative rates in the CPV scenario. The cross-sections for the processes $\mathrm{e}^{+} \mathrm{e}^{-} \rightarrow \mathrm{H}_{i} \mathrm{Z}$ and $\mathrm{e}^{+} \mathrm{e}^{-} \rightarrow \mathrm{H}_{i} \mathrm{H}_{j}$ are given by [28]

$$
\begin{aligned}
\mathrm{e}^{+} \mathrm{e}^{-} \rightarrow \mathrm{H}_{i} \mathrm{Z}: & \sigma_{\mathrm{H}_{i} \mathrm{Z}}=g_{\mathrm{H}_{i} \mathrm{ZZ}}^{2} \sigma_{\mathrm{HZ}}^{\mathrm{SM}}\left(m_{\mathrm{H}_{i}}\right), \\
\mathrm{e}^{+} \mathrm{e}^{-} \rightarrow \mathrm{H}_{i} \mathrm{H}_{j}: & \sigma_{\mathrm{H}_{i} \mathrm{H}_{j}}=g_{\mathrm{H}_{i} \mathrm{H}_{j} \mathrm{Z}}^{2} \bar{\lambda} \sigma_{\mathrm{HZ}}^{\mathrm{SM}}\left(m_{\mathrm{H}_{i}}\right),
\end{aligned}
$$

where the couplings

$$
\begin{aligned}
g_{\mathrm{H}_{i} \mathrm{ZZ}} & =\cos \beta O_{1 i}+\sin \beta O_{2 i} \\
g_{\mathrm{H}_{i} \mathrm{H}_{j} \mathrm{Z}} & =O_{3 i}\left(\cos \beta O_{2 j}-\sin \beta O_{1 j}\right)-O_{3 j}\left(\cos \beta O_{2 i}-\sin \beta O_{1 i}\right)
\end{aligned}
$$

obey the sum rules

$$
\begin{aligned}
\sum_{i=1}^{3} g_{\mathrm{H}_{i} \mathrm{ZZ}}^{2} & =1 \\
g_{\mathrm{H}_{k} \mathrm{ZZ}} & =\frac{1}{2} \sum_{i, j=1}^{3} \varepsilon_{i j k} g_{\mathrm{H}_{i} \mathrm{H}_{j} \mathrm{Z}} .
\end{aligned}
$$

The orthogonal matrix $O_{i j}(i, j=1,2,3)$ relating the weak eigenstates to the mass eigenstates has non-zero off-diagonal elements only in a CPV scenario.

\section{Signal topologies and experimental searches}

The topological searches are devised to detect Higgs boson production via the Higgsstrahlung and pair production processes. Many of these searches are described in earlier publications. Here we emphasize the most recent searches, optimized at the highest LEP energies and some modifications of the earlier searches to increase the sensitivity in parts of the MSSM parameter space which were not sufficiently covered before. The topological searches for the Higgsstrahlung process are listed in Table 1, and those for the pair production process in Table 2 .

In the following, the notation $\mathrm{e}^{+} \mathrm{e}^{-} \rightarrow \mathcal{H Z}$ and $\mathrm{e}^{+} \mathrm{e}^{-} \rightarrow \mathcal{H}_{1} \mathcal{H}_{2}$ is used as a generic notation designating both the CPC processes $\mathrm{e}^{+} \mathrm{e}^{-} \rightarrow \mathrm{hZ}, \mathrm{e}^{+} \mathrm{e}^{-} \rightarrow \mathrm{HZ}$ and $\mathrm{e}^{+} \mathrm{e}^{-} \rightarrow \mathrm{hA}$, and the CPV processes $\mathrm{e}^{+} \mathrm{e}^{-} \rightarrow \mathrm{H}_{1} \mathrm{Z}, \mathrm{e}^{+} \mathrm{e}^{-} \rightarrow \mathrm{H}_{2} \mathrm{Z}$ and $\mathrm{e}^{+} \mathrm{e}^{-} \rightarrow \mathrm{H}_{1} \mathrm{H}_{2}$. Since in $\mathrm{e}^{+} \mathrm{e}^{-}$collisions the kinematic properties of the CPC and CPV signal processes are expected to be very similar, the topological searches described in the following can be applied in both scenarios. In both CPC and CPV models, the production process in Higgsstrahlung only contains couplings between the $\mathrm{Z}$ boson and a CP-even Higgs state, and the pair production always involves a CP-even and a CP-odd state. The production angle distributions are therefore expected to be the same. In CPV models, the Higgs mass eigenstate is a mixture of CP eigenstates and is able to change from a CP-even to a CP-odd state. Even in this case, the Higgs decay angle distributions will stay the same since both the $\mathrm{CP}$-odd and the CP-even states are spin 0 bosons. Small differences not measurable at LEP may arise from different spin correlations in the decay products of the Higgs boson.

\subsection{Searches for Higgsstrahlung processes}

(a) The most efficient search for the Higgsstrahlung processes is the one which is designed to search for the Standard Model Higgs boson. This search [7] is interpreted 
here as a generic search for the corresponding MSSM process $\mathrm{e}^{+} \mathrm{e}^{-} \rightarrow \mathcal{H Z}$. It takes advantage of the preferential decay of Higgs bosons into b $\bar{b}$ and $\tau^{+} \tau^{-}$pairs and addresses the following $\mathrm{Z}$ boson decays: $\mathrm{Z} \rightarrow \mathrm{q} \overline{\mathrm{q}}, \nu \bar{\nu}, \mathrm{e}^{+} \mathrm{e}^{-}, \mu^{+} \mu^{-}, \tau^{+} \tau^{-}$. Moreover, this search is also sensitive to contributions to the signal from the $\mathrm{W}^{+} \mathrm{W}^{-}$and $\mathrm{ZZ}$ fusion processes $\mathrm{e}^{+} \mathrm{e}^{-} \rightarrow \mathcal{H} \nu \bar{\nu}$ and $\mathcal{H} \mathrm{e}^{+} \mathrm{e}^{-}$, which may become important at the kinematic limit of the Higgsstrahlung process.

(b) The Higgs cascade decay $\mathcal{H}_{2} \rightarrow \mathcal{H}_{1} \mathcal{H}_{1}$ may play an important role in regions of the MSSM parameter space where it is kinematically accessible. In order to increase the sensitivity to cascade decays, the search described in 7] is adapted, in those parts which deal with the "four-jet" final state $\mathrm{e}^{+} \mathrm{e}^{-} \rightarrow(\mathcal{H} \rightarrow \mathrm{b} \overline{\mathrm{b}})(\mathrm{Z} \rightarrow \mathrm{q} \overline{\mathrm{q}})$ and the "missing energy" final state $\mathrm{e}^{+} \mathrm{e}^{-} \rightarrow(\mathcal{H} \rightarrow \mathrm{b} \bar{b})(\mathrm{Z} \rightarrow \nu \bar{\nu})$. These searches modified for $\mathrm{e}^{+} \mathrm{e}^{-} \rightarrow\left(\mathcal{H}_{2} \rightarrow \mathcal{H}_{1} \mathcal{H}_{1}\right) \mathrm{Z}$ are described below in Sections 4.1.1 and 4.1.2.

(c) The search for Higgs cascade decays is complemented by an earlier search for $\mathrm{e}^{+} \mathrm{e}^{-} \rightarrow\left(\mathcal{H}_{2} \rightarrow \mathcal{H}_{1} \mathcal{H}_{1}\right) \mathrm{Z}[29$, which is specifically designed to be efficient in the domain $m_{\mathcal{H}_{1}}<10 \mathrm{GeV}$.

(d) For Higgs bosons produced in Higgsstrahlung $\mathrm{e}^{+} \mathrm{e}^{-} \rightarrow \mathcal{H Z}$ and decaying into particles other than b-quarks or $\tau$ leptons, a flavour-independent search for $\mathrm{e}^{+} \mathrm{e}^{-} \rightarrow(\mathcal{H} \rightarrow$ hadrons $) \mathrm{Z}$ [30, 31] is used.

\subsubsection{Modification of the search in the four-jet channel}

The search for the SM Higgs boson in the channel $\mathrm{e}^{+} \mathrm{e}^{-} \rightarrow \mathrm{H}_{\mathrm{SM}} \mathrm{Z} \rightarrow \mathrm{b} \overline{\mathrm{b}} \mathrm{q} \overline{\mathrm{q}}$ [7] is modified to be sensitive to the cascade decay $\mathcal{H}_{2} \rightarrow \mathcal{H}_{1} \mathcal{H}_{1}$. The event selection is identical to the SM search, and thus the same candidate events are observed with the same expected background. The whole event is forced into four jets using the Durham jet finder 32 . If $\mathcal{H}_{1}$ is not too heavy, the two jets from $\mathcal{H}_{1} \rightarrow \mathrm{b} \bar{b}$ are often joined into one jet. The SM four-jet search is therefore also efficient for this decay and the expected signal rates from $\mathrm{e}^{+} \mathrm{e}^{-} \rightarrow\left(\mathcal{H}_{2} \rightarrow \mathrm{b} \overline{\mathrm{b}}\right) \mathrm{Z}$ and $\mathrm{e}^{+} \mathrm{e}^{-} \rightarrow\left(\mathcal{H}_{2} \rightarrow \mathcal{H}_{1} \mathcal{H}_{1} \rightarrow \mathrm{b} \overline{\mathrm{b}} \bar{b}\right) \mathrm{Z}$ can simply be added. The efficiencies for various combinations of $\left(m_{\mathcal{H}_{1}}, m_{\mathcal{H}_{2}}\right)$ are given in Table 3 . The shape of the distribution of the discriminating variable $\mathcal{D}$ [7], however, differs for the two decay modes. $\mathcal{D}$ is a product of a mass independent and a mass dependent likelihood. Depending on $m_{\mathcal{H}_{1}}$, the mass reconstruction is diluted by wrong jet pairings inside one jet, and thus the likelihood distributions are broadened. The signal distribution of $\mathcal{D}$ is therefore constructed at each point of the MSSM parameter space, taking into account the changing relative contributions from the two decays by first adding the relative contributions of $\mathcal{H}_{2} \rightarrow \mathrm{b} \bar{b}$ and $\mathcal{H}_{2} \rightarrow \mathrm{b} \bar{b} b \bar{b}$ in the two likelihoods and then calculating the product. The systematic uncertainties are essentially the same as for the SM channel $\mathrm{e}^{+} \mathrm{e}^{-} \rightarrow \mathrm{H}_{\mathrm{SM}} \mathrm{Z} \rightarrow \mathrm{b} \overline{\mathrm{b}} \mathrm{q} \overline{\mathrm{q}}$ [7].

\subsubsection{Modification of the search in the missing energy channel}

For the data taken at $\sqrt{s}=199$ to $209 \mathrm{GeV}$, the Artificial Neural Network (ANN) analysis for $\mathrm{e}^{+} \mathrm{e}^{-} \rightarrow \mathrm{H}_{\mathrm{SM}} \mathrm{Z} \rightarrow \mathrm{b} \overline{\mathrm{b}} \nu \bar{\nu}$ [7] is reoptimized for $100<m_{\mathcal{H}_{2}}<110 \mathrm{GeV}$ and modified to be sensitive to $\mathcal{H}_{2} \rightarrow \mathrm{b} \overline{\mathrm{b}}$ and $\mathcal{H}_{2} \rightarrow \mathcal{H}_{1} \mathcal{H}_{1} \rightarrow \mathrm{b} \bar{b} \bar{b}$ decays simultaneously. In this mass range, the $\mathcal{H}_{2} \rightarrow \mathcal{H}_{1} \mathcal{H}_{1}$ decay is crucial especially in the CPV scenario.

In the preselection, the event sample is split into two subsamples, according to the 2-to-3 jet resolution parameter $y_{32}$ of the Durham jet finder. Subsample A contains events 
with $y_{32}<0.05\left(\mathcal{H}_{2} \rightarrow \mathrm{b} \bar{b}\right.$ events and most of the $\mathcal{H}_{2} \rightarrow \mathcal{H}_{1} \mathcal{H}_{1} \rightarrow \mathrm{b} \bar{b} b \bar{b}$ with light $\left.\mathcal{H}_{1}\right)$ and subsample $\mathrm{B}$ events with $y_{32} \geq 0.05$ (most of the $\mathcal{H}_{2} \rightarrow \mathcal{H}_{1} \mathcal{H}_{1} \rightarrow \mathrm{b} \bar{b} b \bar{b}$ events with heavier $\mathcal{H}_{1}$ ). The separating value of 0.05 is chosen so that the efficiency for $\mathcal{H}_{2} \rightarrow \mathrm{b} \bar{b}$ events in subsample A is approximately the same as in the SM search. Separate neural networks, $\mathrm{ANN}_{\mathrm{A}}$ and $\mathrm{ANN}_{\mathrm{B}}$ are then trained for events belonging to the two subsamples. For $\mathrm{ANN}_{\mathrm{A}}$ the training is based on $\mathcal{H}_{2} \rightarrow \mathcal{H}_{1} \mathcal{H}_{1} \rightarrow \mathrm{b} \bar{b} b \bar{b}$ signal events with $m_{\mathcal{H}_{1}}=12 \mathrm{GeV}$ and $100 \leq m_{\mathcal{H}_{2}} \leq 110 \mathrm{GeV}$, while for $\mathrm{ANN}_{\mathrm{B}} m_{\mathcal{H}_{1}}=40 \mathrm{GeV}$ and $100 \leq m_{\mathcal{H}_{2}} \leq 110 \mathrm{GeV}$ is used. The analysis is slightly different from the SM missing energy analysis [7.

The preselection cuts 1 to 5 are identical to those described in [7. They are designed to remove accelerator-related backgrounds (such as beam-gas interactions and instrumental noise), dilepton final states, two-photon processes and radiative $\mathrm{q} \overline{\mathrm{q}}$ events, and to select events with a significant amount of missing energy. The following new preselection cuts are applied:

6. The tracks and clusters in the event are grouped into jets using the Durham algorithm. Depending on $y_{32}$, the event is either grouped into two jets $\left(y_{32}<0.05\right)$ in subsample A or into four jets $\left(y_{32} \geq 0.05\right)$ in subsample B. Each event in subsample $\mathrm{B}$ is required to have at least one track per jet.

Additional requirements are imposed for subsample $\mathrm{A}\left(y_{32}<0.05\right)$ :

7. The acoplanarity angle $\left(180^{\circ}\right.$ minus the angle between the two jets when projected into the $x y$ plane) must be between $3^{\circ}$ and $100^{\circ}$ to reject $\mathrm{Z} / \gamma \rightarrow \mathrm{q} \overline{\mathrm{q}}$ events with back-to-back jets.

8. To reduce the background from $\mathrm{W}^{+} \mathrm{W}^{-}$events, the event must not have an identified isolated lepton [10].

The effects of the cuts on the data, the simulated signal and background samples are given in Table 4. The 12 (11) variables used as inputs to $\mathrm{ANN}_{\mathrm{A}}\left(\mathrm{ANN}_{\mathrm{B}}\right)$ are listed below. All variables are scaled to values between zero and one, and in some cases the logarithm of the variable is used, which gives less peaked distributions and is therefore better suited to an ANN analysis. The distributions of some of these input variables are shown in Figs. 1 and 2. The following 9 variables are common to both $\mathrm{ANN}_{\mathrm{A}}$ and $\mathrm{ANN}_{\mathrm{B}}$.

1. The scaled effective centre-of-mass energy [33] $\sqrt{s^{\prime}} / \sqrt{s}$,

2. The scaled missing mass $m_{\text {miss }} / \sqrt{s}$,

3. The polar angle of the missing momentum vector $\left|\cos \theta_{\text {miss }}\right|$,

4. The b-tag likelihood output $\mathcal{B}_{1}[8]$ of the first (highest energy) jet,

5. The b-tag likelihood output $\mathcal{B}_{2}$ of the second highest energy jet,

6. The angle between the first jet and the missing momentum vector, $\ln \left(1-\cos \angle\left(j_{1}, p_{\text {miss }}\right)\right)$,

7. The angle between the second jet and the missing momentum vector, $\cos \angle\left(j_{2}, p_{\text {miss }}\right)$,

8. The $\chi^{2}$ of the one-constraint kinematic fit, $\ln \left(\chi_{\mathrm{HZ}}^{2}\right)$, where the missing mass $m_{\text {miss }}$ is forced to $m_{\mathrm{Z}}$, 
9. The scaled missing momentum $p_{\text {miss }} / \sqrt{s}$.

The following three variables are used in the construction of $\mathrm{ANN}_{\mathrm{A}}$

10. The polar angle of the thrust axis $\left|\cos \theta_{\mathrm{thr}}\right|$,

11. The acoplanarity angle of the jets $\ln \left(\phi_{\text {acop }}\right)$,

12. The logarithm of the energy difference between the two jets $\ln \left|E_{1}-E_{2}\right|$.

The following two variables are used in the construction of $\mathrm{ANN}_{\mathrm{B}}$

10. The b-tag likelihood output $\mathcal{B}_{3}$ of the third jet,

11. The b-tag likelihood output $\mathcal{B}_{4}$ of the fourth jet.

The Higgs mass is reconstructed using the di-jet invariant mass after the 1 constraint kinematic fit. The distributions of the $\mathrm{ANN}_{\mathrm{A}}$ and $\mathrm{ANN}_{\mathrm{B}}$ output variables are shown in Fig. 3, and the distributions of the reconstructed mass in Fig. 4 . Candidate events are selected if $\mathrm{ANN}_{\mathrm{A}}>0.5$ for events in subsample $\mathrm{A}$ and $\mathrm{ANN}_{\mathrm{B}}>0.5$ in subsample $\mathrm{B}$. The efficiencies for signal events for both $\mathcal{H}_{2} \rightarrow \mathrm{b} \bar{b}$ and $\mathcal{H}_{2} \rightarrow \mathcal{H}_{1} \mathcal{H}_{1} \rightarrow \mathrm{b} \bar{b} b \bar{b}$ are determined for both selections. In each point of the MSSM parameter space, the expected signal distributions in the output variables are added from both signal sources according to their expected rates. The number of candidate events in subsample $A(B)$ is $11(8)$ with 10.0 (7.2) events expected from background (see Table 4). The signal efficiencies are shown in Table 5 for various values of $\left(m_{\mathcal{H}_{2}}, m_{\mathcal{H}_{1}}\right)$. The reconstructed masses and the ANN outputs are used to construct the discriminating variable $\mathcal{D}$, which is used in the statistical combination with other search channels.

The systematic uncertainties for this channel are evaluated by analogy to the SM missing energy search [7]. They amount to $2.2 \%$ (9.0\%) in the signal (background) for subsample A and to $2.5 \%(19.3 \%)$ in subsample B. The strongest contributions arise from the uncertainty of the B hadron fragmentation and from Monte Carlo statistics. Systematic uncertainties of $9 \%$ for the background and $1 \%$ for the signal are added in quadrature to account for the uncertainty introduced when simulating the separation of the events into the two subsamples. These are estimated by shifting the value of $y_{32}$ of each event by the difference in the mean values of the background and the data distributions of $y_{32}$ and repeating the selection with the modified value of $y_{32}$.

\subsection{Searches for pair production processes}

(a) The search for the four-b final state $\mathrm{e}^{+} \mathrm{e}^{-} \rightarrow\left(\mathcal{H}_{1} \rightarrow \mathrm{b} \overline{\mathrm{b}}\right)\left(\mathcal{H}_{2} \rightarrow \mathrm{b} \overline{\mathrm{b}}\right)$ provides the highest sensitivity. While in the CPC scenario the pair production process is dominant only for $m_{\mathcal{H}_{1}} \approx m_{\mathcal{H}_{2}}$, this is not the case in the CPV scenario. The search in this channel is therefore optimized separately for small $m_{\mathcal{H}_{1}}$ and large $m_{\mathcal{H}_{1}}$. These are described below in Sections 4.2.1 and 4.2.2.

(b) For the Higgs cascade decay $\mathrm{e}^{+} \mathrm{e}^{-} \rightarrow\left(\mathcal{H}_{1} \rightarrow \mathrm{bb}\right)\left(\mathcal{H}_{2} \rightarrow \mathcal{H}_{1} \mathcal{H}_{1} \rightarrow \mathrm{b} \bar{b} b \bar{b}\right)$ with 6 b-quarks in the final state, the search in the four-b final state for similar masses described in Section 4.2.1 is used because it has a reasonably good efficiency. Even for large mass differences and thus small $m_{\mathcal{H}_{1}}$ this search is more efficient than the one described in Section 4.2.2 due to the highly spherical shape of the six-b events. This search is described in Section 4.2.3 
(c) The search for the final states $\mathrm{e}^{+} \mathrm{e}^{-} \rightarrow\left(\mathcal{H}_{1} \rightarrow \mathrm{b} \bar{b}\right)\left(\mathcal{H}_{2} \rightarrow \tau^{+} \tau^{-}\right)$and $\mathrm{e}^{+} \mathrm{e}^{-} \rightarrow\left(\mathcal{H}_{1} \rightarrow \tau^{+} \tau^{-}\right)\left(\mathcal{H}_{2} \rightarrow \mathrm{b} \overline{\mathrm{b}}\right)$ follow the technique described in [7] for the corresponding Standard Model channels. The final likelihood selection and its optimization for the MSSM case is described in Section 4.2.4

\subsubsection{Search for $\mathrm{e}^{+} \mathrm{e}^{-} \rightarrow \mathcal{H}_{1} \mathcal{H}_{2} \rightarrow \mathrm{b} \bar{b} b \bar{b}$ optimized for high $m_{\mathcal{H}_{1}}$}

Events from the process $\mathcal{H}_{1} \mathcal{H}_{2} \rightarrow \mathrm{b} \bar{b} b \bar{b}$ with high $m_{\mathcal{H}_{1}}$ have four energetic b-jets and a total visible energy close to the centre-of-mass energy. The dominant backgrounds arise from the four-fermion processes $\mathrm{e}^{+} \mathrm{e}^{-} \rightarrow \mathrm{ZZ}$ and $\mathrm{e}^{+} \mathrm{e}^{-} \rightarrow \mathrm{W}^{+} \mathrm{W}^{-}$and from two-fermion processes $\mathrm{e}^{+} \mathrm{e}^{-} \rightarrow \mathrm{q} \overline{\mathrm{q}}(\gamma)$. The events are forced into four jets using the Durham jet finding algorithm and the following preselection is applied.

1. The event must qualify as a multi-hadronic final state according to [33],

2. The effective centre-of-mass energy $\sqrt{s^{\prime}}$ is required to be higher than $0.794 \sqrt{s}$,

3. The 3-to-4 jet resolution parameter $y_{43}$ [32] is required to be larger than 0.003 ,

4. The $C$-Parameter [34, which is a measure of the spherical shape of the event, is required to be larger than 0.45 ,

5. The sum of the number of reconstructed tracks and electromagnetic clusters not associated to tracks [35] belonging to each jet has to be larger than six,

6. To discriminate against poorly reconstructed events, a 4-constraint kinematic fit is applied, using energy and momentum conservation; this fit is required to converge and the $\chi^{2}$ probability is required to be larger than $10^{-5}$.

For the events passing the preselection, a likelihood function is constructed from seven input variables, allowing the events to be classified as signal or background. The likelihood variables are:

1. The four b-tagging discriminants $\mathcal{B}_{i}$ [8] for each of the four jets, ordered by energy,

2. The logarithm of the jet resolution parameter $y_{43}$,

3. The event thrust value $T$,

4. The estimate of the $\mathcal{H}_{1} \mathcal{H}_{2}$ production angle, $\left|\cos \theta_{\text {dijet }}\right|$, which is defined as follows. For the jet pairing that yields the smallest difference between the two dijet-masses, $\left|\cos \theta_{\text {dijet }}\right|$ is the absolute value of the cosine of the dijet polar angle.

The signal reference histograms are obtained using Monte Carlo samples with $m_{\mathcal{H}_{1}} \geq$ $60 \mathrm{GeV}$ and $m_{\mathcal{H}_{2}} \geq 60 \mathrm{GeV}$. For the background, reference histograms are formed from $\mathrm{e}^{+} \mathrm{e}^{-} \rightarrow \mathrm{q} \overline{\mathrm{q}}(\gamma)$ and $\mathrm{e}^{+} \mathrm{e}^{-} \rightarrow \mathrm{q} \overline{\mathrm{q}} \mathrm{q} \overline{\mathrm{q}}$ events. The distributions of these variables are shown in Figure 5. Events are selected if they satisfy $\mathcal{L}>0.95$, which provides the best sensitivity measured in terms of $s / \sqrt{b+2}$ for $m_{\mathcal{H}_{1}}=m_{\mathcal{H}_{2}}=90 \mathrm{GeV}$.

The numbers of observed and expected background events after each preselection cut and the final likelihood cut are shown in Table 6 for data taken at $\sqrt{s}=192$ to $209 \mathrm{GeV}$. The distribution of the likelihood output is shown in Figure 7 (a). The efficiencies for various combinations of $\left(m_{\mathcal{H}_{1}}, m_{\mathcal{H}_{2}}\right)$ are shown in Table 7. 
The mass of the Higgs boson candidates is reconstructed using a constrained fit requiring energy and momentum conservation. Figures 8 (a)-(c) show the distributions of the sum of the reconstructed Higgs boson masses, $M_{\text {sum }}=m_{\mathcal{H}_{1}}^{\text {rec }}+m_{\mathcal{H}_{2}}^{\text {rec }}$, for the jet combination with the largest, second largest and smallest value for $\left|m_{\mathcal{H}_{1}}^{\text {rec }}-m_{\mathcal{H}_{2}}^{\text {rec }}\right|$. No significant excess over the expected background is observed. The discriminating variable $\mathcal{D}$ is a two-dimensional array of reconstructed masses $m_{\mathcal{H}_{2}}^{\mathrm{rec}}+m_{\mathcal{H}_{1}}^{\mathrm{rec}}$ and $m_{\mathcal{H}_{2}}^{\mathrm{rec}}-m_{\mathcal{H}_{1}}^{\mathrm{rec}}$.

The systematic uncertainties on the signal efficiencies and background expectation for the $\mathcal{H}_{1} \mathcal{H}_{2} \rightarrow \mathrm{b} \bar{b} b \bar{b}$ search are given in Table 8 . They are evaluated as for the SM searches in [7] and include Monte Carlo statistics (uncorrelated between channels, energies and signal and background), detector modelling, such as tracking resolution in $r \phi$ and $z$, hit matching efficiency in the silicon microvertex detector for $r \phi$ and $z$, B-hadron decay multiplicity and momentum spectrum, c-hadron momentum spectrum, comparison between different SM Monte Carlo generators, uncertainties of the four-fermion crosssection (all taken to be fully correlated between channels and energies) and uncertainties in the modelling of the likelihood variables (taken to be uncorrelated between channels and fully correlated between energies of the same channel). The systematic uncertainty amounts to $3.1 \%$ for the signal and $10.3 \%$ for the background expectation.

\subsubsection{Search for $\mathrm{e}^{+} \mathrm{e}^{-} \rightarrow \mathcal{H}_{1} \mathcal{H}_{2} \rightarrow \mathrm{b} \overline{\mathrm{b}} \mathrm{b} \overline{\mathrm{b}}$ for low $\boldsymbol{m}_{\mathcal{H}_{1}}$}

The region $12 \mathrm{GeV}<m_{\mathcal{H}_{1}}<30 \mathrm{GeV}$ and $m_{\mathcal{H}_{2}}>90 \mathrm{GeV}$ is of particular interest in the CPV scenario. The following selection is optimized for that kinematic region and replaces the selection of Section 4.2.1. Events with large $m_{\mathcal{H}_{2}}-m_{\mathcal{H}_{1}}$ look like asymmetrically boosted three-jet events.

The preselection is identical to the one of Section 4.2.1 except for cuts (3) and (4). In (3) the cut on $y_{43}$ is relaxed to 0.0003. In order to compensate for the increased $q \bar{q} \gamma$-background an additional requirement is introduced in cut (3): the sum of the two smallest angles between any jets, $J_{2}$, has to satisfy the requirement $30^{\circ}<J_{2}<175^{\circ}$ and the sum of the four smallest angles between jets, $J_{4}$, has to satisfy $220^{\circ}<J_{4}<400^{\circ}$. Cut (4) is relaxed to $C>0.2$. This increases the acceptance for asymmetric three-jet-like events. The number of selected events after each cut, along with the expected background, is shown in Table 9 .

After the preselection, a likelihood function is constructed from the seven variables described in Section 4.2.1. Signal reference histograms are formed from Monte Carlo samples with $12<m_{\mathcal{H}_{1}}<30 \mathrm{GeV}$ and $90<m_{\mathcal{H}_{2}}<110 \mathrm{GeV}$. The distributions of the input variables are shown in Fig. 6. The resulting likelihood distribution is shown in Fig. Z7(b). The cut $\mathcal{L}>0.98$ is applied, which is optimal for $m_{\mathcal{H}_{1}}=30 \mathrm{GeV}$ and $m_{\mathcal{H}_{2}}=100 \mathrm{GeV}$.

The efficiencies for various combinations of $\left(m_{\mathcal{H}_{1}}, m_{\mathcal{H}_{2}}\right)$ are shown in Table 10, The distribution of the reconstructed mass sum $M_{\text {sum }}$ is shown in Fig. 9, No significant excess over the background is observed. The discriminating variable $\mathcal{D}$ is a two-dimensional array

of reconstructed masses $m_{\mathcal{H}_{2}}^{\text {rec }}+m_{\mathcal{H}_{1}}^{\text {rec }}$ and $m_{\mathcal{H}_{2}}^{\text {rec }}-m_{\mathcal{H}_{1}}^{\text {rec }}$. The systematic uncertainties are listed in Table 8 and are derived in the same way as for the search described in Section 4.2.1. They amount to $4.7 \%$ for the signal and $10.5 \%$ for the background expectation.

\subsubsection{Search for $\mathrm{e}^{+} \mathrm{e}^{-} \rightarrow \mathcal{H}_{1} \mathcal{H}_{2} \rightarrow \mathcal{H}_{1} \mathcal{H}_{1} \mathcal{H}_{1} \rightarrow \mathrm{b} \overline{\mathrm{b}} \mathrm{b} \overline{\mathrm{b}} \mathrm{b} \overline{\mathrm{b}}$}

The search channel for $\mathcal{H}_{1} \mathcal{H}_{2} \rightarrow \mathrm{b} \bar{b} b \bar{b}$ optimized for high $m_{\mathcal{H}_{1}}$ is also used to search for events of the type $\mathrm{e}^{+} \mathrm{e}^{-} \rightarrow \mathcal{H}_{1} \mathcal{H}_{2} \rightarrow \mathcal{H}_{1} \mathcal{H}_{1} \mathcal{H}_{1} \rightarrow \mathrm{bb} \bar{b} \bar{b} \bar{b}$. Despite the large mass difference 
$m_{\mathcal{H}_{2}}-m_{\mathcal{H}_{1}}$ and generally relatively low $m_{\mathcal{H}_{1}}$, the selection for high $m_{\mathcal{H}_{1}}$ (Section 4.2.1) is more efficient than the selection for low $m_{\mathcal{H}_{1}}$ (Section 4.2.2) due to the spherical shape

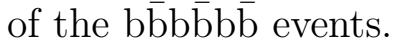

The expected signal distribution is added to the one from the $4 \mathrm{~b}$-channel in the same way as described in Section 4.1.1. The efficiency of this search is shown in Table 11] for various $\left(m_{\mathcal{H}_{1}}, m_{\mathcal{H}_{2}}\right)$. The systematic error of the signal of this channel is taken to be the same as for the search optimized for high $m_{\mathcal{H}_{1}}$.

\subsubsection{Search for the process $\mathrm{e}^{+} \mathrm{e}^{-} \rightarrow \mathcal{H}_{1} \mathcal{H}_{2} \rightarrow \mathrm{b} \overline{\mathrm{b}} \tau^{+} \tau^{-}$and $\tau^{+} \tau^{-} \mathrm{b} \overline{\mathrm{b}}$}

The search for the $\mathcal{H}_{1} \mathcal{H}_{2} \rightarrow \mathrm{b} \overline{\mathrm{b}} \tau^{+} \tau^{-}$final state, where either $\mathcal{H}_{1}$ or $\mathcal{H}_{2}$ decays into a tau pair, uses the same techniques as the SM tau search [7. The final likelihood selection including the b-tagging is optimized for the MSSM process. The likelihood $\mathcal{L}_{\mathcal{H}_{1} \mathcal{H}_{2}}$ differs from the SM likelihood in the following way.

1. The signal reference histograms are constructed from simulated $\mathcal{H}_{1} \mathcal{H}_{2}$ events, using a large range of $m_{\mathcal{H}_{1}}$ and $m_{\mathcal{H}_{2}}$ values,

2. The $\chi^{2}$ probability of the 3 -constraint fit, constraining the invariant mass of the $\tau^{+} \tau^{-}$pair to $m_{\mathrm{Z}}$, is dropped,

3. The variable $\left|\cos \theta_{\text {dijet }}\right|$ (cf. Section 4.2.1) is introduced. Here the dijet pairing is defined by the pair of b-tagged jets and the pair of tau jets. The mean value of the cosines of the two systems is taken.

The distributions of the likelihood input variables are shown in Fig. 10, The distribution of the likelihood $\mathcal{L}$ is shown in Fig. 11, and the number of selected events after each cut along with the expected background is shown in Table 6 . The discriminating variable $\mathcal{D}$ is a twodimensional array of reconstructed masses $m_{\mathcal{H}_{2}}^{\text {rec }}+m_{\mathcal{H}_{1}}^{\text {rec }}$ and $m_{\mathcal{H}_{2}}^{\text {rec }}-m_{\mathcal{H}_{1}}^{\text {rec }}$. The cut $\mathcal{L}>0.64$ is applied. The efficiencies of the selection are given in Table 12. Systematic uncertainties are listed in Table 8. They are derived in the same way as in Section 4.2.1. Additionally the systematic error on the tau identification is evaluated using event mixing 7 . They amount to $2.6 \%$ for the signal and $15.8 \%$ for the background expectation.

\subsection{Comparison of Data and Expected Background Monte Carlo}

In Table 13 the results of the new searches, which are described in detail in this section, are summarized in terms of total background and data events. There is good agreement between the selected data events and the expected SM background. The numbers in this table are obtained by choosing for illustration $m_{\mathcal{H}_{1}}=39 \mathrm{GeV}$ and $m_{\mathcal{H}_{2}}=105 \mathrm{GeV}$ in those searches where the selections depend explicitly on the hypothetical Higgs boson masses.

No significant excess of candidate events over the expected background is found in any of the old and new search channels. 


\section{Combination of search channels and hypothesis testing}

\subsection{Combined confidence levels}

The sensitivity of the searches for hypothetical Higgs bosons is increased by combining the results of the various topological searches. This is done following the statistical method described in [7.

In order to compute confidence levels, a test statistic $Q$ is defined that can be used to quantify the compatibility of the data with two hypotheses: the background hypothesis and the signal+background hypothesis. The confidence levels are computed from a comparison of the observed test statistic and its probability distributions for a large number of simulated experiments for these two hypotheses. In this paper, the ratio $Q=\mathcal{L}_{s+b} / \mathcal{L}_{b}$ of the likelihoods for the two hypotheses is chosen as the test statistic. The results of all search channels are expressed in fine bins of discriminating variables $\mathcal{D}$, as defined in the descriptions of the individual searches. For each bin $i$ of $\mathcal{D}$ three numbers are calculated: $s_{i}$, the number of expected signal events for a given set of model parameters (Higgs masses etc.), $b_{i}$, the number of expected background events, and $n_{i}$, the number of observed events. Each bin is considered to be a statistically independent counting experiment obeying Poisson statistics. The test statistic can then be computed [7] as

$$
-2 \ln Q=2 \sum_{i} s_{i}-2 \sum_{i} n_{i} \ln \left(1+s_{i} / b_{i}\right) .
$$

The confidence level for the background hypothesis, $\mathrm{CL}_{b}$, is defined as the probability to obtain values of $Q$ no larger than the observed value $Q_{\mathrm{obs}}$, given a large number of hypothetical experiments with background processes only,

$$
\mathrm{CL}_{b}=P\left(Q \leq Q_{\text {obs }} \mid \text { background }\right) \text {. }
$$

Similarly, the confidence level for the signal+background hypothesis, $\mathrm{CL}_{s+b}$, is defined as the probability to obtain values of $Q$ smaller than observed, given a large number of hypothetical experiments with signal and background processes,

$$
\mathrm{CL}_{s+b}=P\left(Q \leq Q_{\text {obs }} \mid \text { signal }+ \text { background }\right) .
$$

In principle, $\mathrm{CL}_{s+b}$ can be used to exclude the signal+background hypothesis, given a model for Higgs boson production. However, this procedure may lead to the undesired possibility that a downward fluctuation of the background would allow hypotheses to be excluded for which the experiment has no sensitivity due to the small expected signal rate. This problem is avoided by introducing the ratio

$$
\mathrm{CL}_{s}=\mathrm{CL}_{s+b} / \mathrm{CL}_{b}
$$

Since $\mathrm{CL}_{b}$ is a positive number less than one, $\mathrm{CL}_{s}$ will always be greater than $\mathrm{CL}_{s+b}$ and the limit obtained in this way will therefore be conservative. We adopt this quantity for setting exclusion limits and consider a hypothesis to be excluded at the $95 \%$ confidence level if the corresponding value of $\mathrm{CL}_{s}$ is less than 0.05 .

The expected confidence levels are obtained by replacing the observed data configuration by a large number of simulated event configurations for the two hypotheses background only or signal+background. These can be used to estimate the expected sensitivity 
of a search and to compare the observed exclusion with the one expected with no signal present.

The effect of systematic uncertainties of the individual channels is calculated using a Monte Carlo technique. The signal and background estimations are varied within the bounds of the systematic uncertainties, assuming Gaussian distributions of the uncertainties. Correlations are taken into account. These variations are convoluted with the Poisson statistical variations of the assumed signal and background rates in the confidence level calculation. The effect of systematic uncertainties on the exclusion limits turns out to be generally small.

In case of overlapping channels, i.e. channels sharing a fraction of events, the approach described above is modified. Such a situation occurs for example for the Higgsstrahlung searches with and without b-tagging [7,31] or in the case of the Higgsstrahlung four-jet channel and the pair production four-b channel. We calculate the expected $\mathrm{CL}_{s}$ for each of the overlapping channels in turn, and retain only the channel that yields the smaller expected $\mathrm{CL}_{s}$. This procedure is repeated for each signal hypothesis. For different Higgs boson masses therefore different search channels give the exclusion.

The same procedure is applied if two signal processes, for example $\mathcal{H}_{1} \mathrm{Z}$ and $\mathcal{H}_{2} \mathrm{Z}$, can contribute to the same event topology, but at different mass values. In the case of the four-jet channel the selection procedure and discriminant variable $\mathcal{D}$ depend on the Higgs mass hypothesis (test mass). Two different test masses have not only different signal distributions $s_{i}$ but also different background and data distributions $b_{i}$ and $n_{i}$. The selected events in searches for $\mathcal{H}_{1} \mathrm{Z}$ and for $\mathcal{H}_{2} \mathrm{Z}$ cannot be combined since the inconsistent background and data distributions for the two hypotheses in general contain an overlapping sample of data events. Therefore only the hypothesis that yields the lower expected $\mathrm{CL}_{s}$ is retained.

\subsection{Additional experimental constraints}

If a given model for Higgs production is not excluded by using the search channels described above, the following additional constraints are considered:

(a) The constraint from the measured $\mathrm{Z}$ boson decay width $\Gamma_{\mathrm{Z}}$ : a model is regarded as excluded if the condition

$$
\sum_{i} \sigma_{\mathcal{H}_{i} \mathrm{Z}}(91.4 \mathrm{GeV})+\sum_{i, j} \sigma_{\mathcal{H}_{i} \mathcal{H}_{j}}(91.4 \mathrm{GeV})>\sigma_{\mathrm{Z}}(91.4 \mathrm{GeV}) \frac{\Delta \Gamma_{\mathrm{Z}}}{\Gamma_{\mathrm{Z}}}
$$

is satisfied using results from [36]. The nominal LEP1 centre-of-mass energy of $\sqrt{s}=91.4 \mathrm{GeV}$ is used. $\Gamma_{\mathrm{Z}}$ is the total $\mathrm{Z}$ width, $\sigma_{\mathrm{Z}}$ is the total $\mathrm{Z}$ cross-section and $\Delta \Gamma_{\mathrm{Z}}=6.5 \mathrm{MeV}$ is the maximum additional width that is compatible with the measured width, given the SM hypothesis (obtained from ZFITTER [37]) at the 95\% CL.

(b) The constraint from the decay mode independent search for $\mathrm{e}^{+} \mathrm{e}^{-} \rightarrow \mathrm{HZ}$ [38]: a model is regarded as excluded if

$$
\sigma_{\mathcal{H}_{i} \mathrm{Z}}>k\left(m_{\mathcal{H}_{i}}\right) \sigma_{\mathrm{HZ}}^{\mathrm{SM}}\left(m_{\mathcal{H}_{i}}\right) \quad \text { with } \quad m_{\mathcal{H}_{i}}=m_{\mathrm{H}}
$$

is fulfilled, where $k\left(m_{\mathcal{H}_{i}}\right)$ is the smallest scale factor for the SM Higgs production cross-section that is excluded at the $95 \%$ CL by this search. This criterion is used 
for $\mathcal{H}_{i}=\mathcal{H}_{1}$ and $\mathcal{H}_{i}=\mathcal{H}_{2}$ and at $\sqrt{s}=91.4,183$ and $206 \mathrm{GeV}$. The use of $\mathrm{Z}$ width constraints and decay mode independent analyses is especially helpful for the range $m_{\mathcal{H}_{1}}<6 \mathrm{GeV}$. In the CPV scenarios all points excluded by the Z-width constraint turn out to be also excluded by the decay mode independent search.

(c) The constraint from a search for Yukawa production of a light Higgs boson [39]: a model is regarded as excluded if the predicted value of the Yukawa enhancement factor $\xi$ for $\mathcal{H}_{1}$, multiplied with the branching fraction $\operatorname{BR}\left(\mathcal{H}_{1} \rightarrow \tau^{+} \tau^{-}\right)$, is larger than the smallest value excluded in [39. In the case of the CPV scan, where $\mathcal{H}_{1}$ is composed of CP-odd and CP-even parts, the weaker of the two limits calculated for the Yukawa production of a CP-even or a CP-odd Higgs boson is used in the comparison. For CP-even Higgs bosons, $\xi=-\sin \alpha / \cos \beta$, while for CP-odd Higgs bosons $\xi=\tan \beta$ holds. This constraint is helpful in excluding models with large $\tan \beta, 2 m_{\tau}<m_{\mathcal{H}_{1}}<2 m_{\mathrm{b}}$ and vanishing $\mathrm{e}^{+} \mathrm{e}^{-} \rightarrow \mathcal{H}_{1} \mathrm{Z}$ cross-section.

(d) As an overlay, the constraint from measurements of inclusive decays of a b quark into an $\mathrm{s}$ quark and a photon $\mathrm{BR}(\mathrm{b} \rightarrow \mathrm{s} \gamma)$ is shown. A model is shown as excluded by this constraint if the corresponding branching ratio, calculated using [40], falls

outside the bounds $2.33 \times 10^{-4}<\mathrm{BR}(\mathrm{b} \rightarrow \mathrm{s} \gamma)<4.15 \times 10^{-4}(95 \% \mathrm{CL})$ [41]. This limit is used to constrain the CPC scenarios.

\section{Model-independent limits on topological cross- sections}

For the model-independent interpretation of the OPAL Higgs searches the scaling factor

$$
s_{95}=\frac{\sigma_{\max }}{\sigma_{\text {ref }}}
$$

is computed, where $\sigma_{\max }$ is the largest production cross-section allowed at $95 \% \mathrm{CL}$ and $\sigma_{\text {ref }}$ is a reference cross-section. For Higgsstrahlung the SM cross-section $\sigma_{\mathrm{SM}}$ is used as $\sigma_{\text {ref }}$; for pair production the cross-section of equation (3) with $\cos ^{2}(\beta-\alpha)=1$ is used. Initial-state radiation is included according to 42 .

Cross-section limits on the SM-like production and decay can be found in [7] and for flavour independent $\mathcal{H} \rightarrow \mathrm{q} \overline{\mathrm{q}}$ decays in 31 .

Fig. 12 (a) shows $s_{95}$ for the production process $\mathrm{e}^{+} \mathrm{e}^{-} \rightarrow \mathcal{H}_{2} \mathrm{Z} \rightarrow \mathcal{H}_{1} \mathcal{H}_{1} \mathrm{Z} \rightarrow \mathrm{bb} b \bar{b} \mathrm{Z}$. $\operatorname{BR}\left(\mathcal{H}_{2} \rightarrow \mathcal{H}_{1} \mathcal{H}_{1}\right)=1$ and $\operatorname{BR}\left(\mathcal{H}_{1} \rightarrow \mathrm{bb}\right)=1$ is assumed. The observed borders and discontinuities stem from a number of different searches contributing and being sensitive in different mass ranges. For $m_{\mathcal{H}_{2}}<80 \mathrm{GeV}$, specific searches for this final state at $183 \mathrm{GeV}$ provide a strong exclusion. For $80<m_{\mathcal{H}_{2}}<100 \mathrm{GeV}$, only the $\mathrm{Z} \rightarrow \mathrm{q} \overline{\mathrm{q}}$ final state is used, giving a weaker exclusion. For $100<m_{\mathcal{H}_{2}}<110 \mathrm{GeV}$, the $\mathrm{Z} \rightarrow \nu \bar{\nu}$ final state is also employed. The limits are calculated for $m_{\mathcal{H}_{1}}>10.5 \mathrm{GeV}$ only where the decay $\mathcal{H}_{1} \rightarrow \mathrm{b} \overline{\mathrm{b}}$ becomes kinematically possible.

Fig. 12 (b) shows $s_{95}$ for the process $\mathrm{e}^{+} \mathrm{e}^{-} \rightarrow \mathcal{H}_{1} \mathcal{H}_{2} \rightarrow \mathrm{b} \bar{b} b \bar{b} . \quad \operatorname{BR}\left(\mathcal{H}_{1} \rightarrow \mathrm{b} \bar{b}\right)=$ $\operatorname{BR}\left(\mathcal{H}_{2} \rightarrow \mathrm{b} \bar{b}\right)=1$ is assumed. The kinematic limit for $\sqrt{s}=206 \mathrm{GeV}$ is indicated as a dashed line. Most searches apply only for $m_{\mathcal{H}_{1}}>30 \mathrm{GeV}$. Below $m_{\mathcal{H}_{1}}=30 \mathrm{GeV}$, only searches for pair production at $\sqrt{s}=183 \mathrm{GeV}$ or lower contribute. Additionally, the area of $m_{\mathcal{H}_{1}}>12 \mathrm{GeV}$ and $90<m_{\mathcal{H}_{2}}<110 \mathrm{GeV}$ is studied in the data at $\sqrt{s}=199$ to $209 \mathrm{GeV}$. 
In Fig. 13 (a) $s_{95}$ for the process $\mathrm{e}^{+} \mathrm{e}^{-} \rightarrow \mathcal{H}_{1} \mathcal{H}_{2} \rightarrow \mathrm{b} \overline{\mathrm{b}} \tau^{+} \tau^{-}$is shown. The branching ratios are set to $\operatorname{BR}\left(\mathcal{H}_{1} \rightarrow \mathrm{b} \bar{b}\right)=\operatorname{BR}\left(\mathcal{H}_{2} \rightarrow \mathrm{b} \bar{b}\right)=0.5$ and $\operatorname{BR}\left(\mathcal{H}_{1} \rightarrow \tau^{+} \tau^{-}\right)=\operatorname{BR}\left(\mathcal{H}_{2} \rightarrow \tau^{+} \tau^{-}\right)=$ 0.5. The kinematic limit for $\sqrt{s}=206 \mathrm{GeV}$ is indicated as a dashed line. The domain below $m_{\mathcal{H}_{1}}=30 \mathrm{GeV}$ is covered only by data collected at $\sqrt{s}=183 \mathrm{GeV}$ or lower.

Fig. 13 (b) shows the exclusion region for the process $\mathrm{e}^{+} \mathrm{e}^{-} \rightarrow \mathcal{H}_{1} \mathcal{H}_{2} \rightarrow \mathcal{H}_{1} \mathcal{H}_{1} \mathcal{H}_{1} \rightarrow \mathrm{bb} \bar{b} \bar{b} \bar{b} . \quad \operatorname{BR}\left(\mathcal{H}_{2} \rightarrow \mathcal{H}_{1} \mathcal{H}_{1}\right)=1$ and $\operatorname{BR}\left(\mathcal{H}_{1} \rightarrow \mathrm{b} \bar{b}\right)=1$ are assumed. At $m_{\mathcal{H}_{2}}<80 \mathrm{GeV}$ the exclusion is stronger than for higher $m_{\mathcal{H}_{2}}$ due to dedicated searches at $\sqrt{s}$ up to $189 \mathrm{GeV}$. Above $m_{\mathcal{H}_{2}}=80 \mathrm{GeV}$, only searches using data recorded with $\sqrt{s}$ of 199 to $209 \mathrm{GeV}$ are available.

\section{Interpretation of the search results in the MSSM}

The presence of neutral Higgs bosons is tested in a constrained MSSM with seven parameters. Two of these parameters are sufficient to describe the Higgs sector at tree level. A convenient choice is $\tan \beta$ (the ratio of the vacuum expectation values of the Higgs fields) and one Higgs mass; $m_{\mathrm{A}}$ is chosen in the case of the CPC scenario and $m_{\mathrm{H}^{ \pm}}$in the CPV scenario. Additional parameters appear at the level of radiative corrections; these are: $m_{\mathrm{SUSY}}, M_{2}, \mu, A$, and $m_{\tilde{\mathrm{g}}}$. All soft SUSY-breaking parameters in the sfermion sector are set to $m_{\mathrm{SUSY}}$ at the electroweak scale. $M_{2}$ is the $\mathrm{SU}(2)$ gaugino mass parameter at the electroweak scale and $M_{1}$, the $\mathrm{U}(1)$ gaugino mass parameter, is derived from $M_{2}$ using the GUT relation $M_{1}=M_{2}\left(5 \sin ^{2} \theta_{W} / 3 \cos ^{2} \theta_{W}\right)$, where $\theta_{W}$ is the weak mixing angle ${ }^{2}$. The supersymmetric Higgs mass parameter is denoted $\mu$. The parameter $A=A_{\mathrm{t}}=A_{\mathrm{b}}$ is the common trilinear Higgs-squark coupling for up-type and down-type squarks. The stop and sbottom mixing parameters are defined as $X_{\mathrm{t}}=A_{\mathrm{t}}-\mu \cot \beta$ and $X_{\mathrm{b}}=A_{\mathrm{b}}-\mu \tan \beta$. The parameter $m_{\tilde{\mathrm{g}}}$ is the gluino mass. For the CPV scenario the complex phases related to $A_{\mathrm{t}, \mathrm{b}}$ and $m_{\tilde{\mathrm{g}}}$ are additional parameters. The phase related to $A_{\mathrm{t}, \mathrm{b}}$ enters at one-loop level while the one related to $m_{\tilde{\mathrm{g}}}$ enters as a second-order correction to stop and sbottom loops. Large radiative corrections to the predicted mass $m_{\mathcal{H}_{1}}$ arise from scalar top loops, while the contributions from scalar bottom loops are smaller.

The precise mass of the top quark has a strong impact on $m_{\mathcal{H}_{1}}$; it is taken to be $m_{\text {top }}=174.3 \mathrm{GeV}$, the current average of the Tevatron measurements [43]. To account for the current experimental uncertainty, all MSSM interpretations are also done for $m_{\text {top }}=169 \mathrm{GeV}$ and $m_{\text {top }}=179 \mathrm{GeV}$.

Rather than varying all of the above MSSM parameters independently, we consider only a certain number of "benchmark sets" where the tree level parameters $\tan \beta$ and $m_{\mathrm{A}}$ (CPC scenario) or $m_{\mathrm{H}^{ \pm}}$(CPV scenario) are scanned while all other parameters are fixed. Results are presented for eight benchmark sets [44,16] in the CPC scenario and nine in the CPV scenario [28]. Each scan point within a given benchmark set defines an independent realization of the MSSM (a model), which is tested by comparing its predicted observables (masses, cross-sections and decay branching ratios) with the experimental data. The parameters of the scans are summarized in Table 14.

For a given scan point the observables in the Higgs sector are calculated using two theoretical approaches. The FEYNHIGGS program [45,46] is based on a two-loop diagrammatic approach [47,48, and uses the OS renormalization scheme, while SUBHPOLE and its CPV variant $\mathrm{CPH}$ [28] are based on a one-loop renormalization group improved

\footnotetext{
${ }^{2} M_{3}, M_{2}$ and $M_{1}$ are the mass parameters associated with the $\mathrm{SU}(3), \mathrm{SU}(2)$ and $\mathrm{U}(1)$ subgroups of the Standard Model. $M_{3}$ enters only via loop corrections sensitive to the gluino mass.
} 
calculation [49, 50, 51, 52 and uses the $\overline{\mathrm{MS}}$ scheme. Both calculations give consistent results although small differences naturally exist. Numerical values for parameters in this paper are given in the $\overline{\mathrm{MS}}$ scheme.

In the CPC case, the FEYNHIGGS calculation is retained for the presentation of the results since it yields slightly more conservative results (the theoretically allowed parameter space is wider) than SUBHPOLE. Also, FEYNHIGGS is preferred on theoretical grounds since its radiative corrections are more detailed than those of SUBHPOLE.

In the CPV case, neither of the two existing calculations is preferred a priori on theoretical grounds. While FEYNHIGGS contains more advanced one-loop corrections, $\mathrm{CPH}$ is more precise at the two-loop level. We therefore opted for a solution where, in each scan point, the calculation yielding the more conservative result (less significant exclusion) is retained. For illustration, the results from FEYNHIGGS and CPH are also shown separately for the main CPV scenario CPX (see Section [7.2).

The limits obtained for the different benchmark sets are summarized in Table 15 .

\subsection{CPC benchmark scenarios}

Of the eight CPC benchmark sets examined in this paper, sets 1, 3 and 6 have been used in the past. Scenarios 4 and 5 are motivated by experimental constraints on the branching ratio of the inclusive decay $\mathrm{b} \rightarrow \mathrm{s} \gamma$ and recent measurements of the muon anomalous magnetic moment $(g-2)_{\mu}$. Benchmark sets 2,7 and 8 are motivated by the fact that the planned Higgs searches at the LHC may have low sensitivity to detect Higgs bosons in these situations. The choice of parameters is summarized in Table 15.

In most cases, $\tan \beta$ is scanned between 0.4 and 40 . For values below 0.4 the theoretical predictions become unreliable; for $\tan \beta$ larger than 40 the decay width of the Higgs bosons may become comparable to or larger than the experimental mass resolution, and the modelling of the signal efficiencies may loose precision. The value of $m_{\mathrm{A}}$ is scanned between 0 and $1000 \mathrm{GeV}$. For values of $m_{\mathrm{A}}<2 \mathrm{GeV}$, the branching ratios of the A become dominated by resonances and their calculation is unstable. However this area can be probed using direct searches for the heavier h boson, decay independent searches and $\Gamma_{\mathrm{Z}}$ constraints.

In general there is good agreement between the data and the background estimation, therefore limits on the MSSM parameters can be derived. In the CPC scenarios, the largest observed excess of the data over the background appears at $m_{\mathrm{h}}=95 \mathrm{GeV}$ in the $m_{\mathrm{h}}-$ max benchmark set. At this point the excess is $\left(1-\mathrm{CL}_{b}\right)=2.6 \times 10^{-3}$, corresponding to a significance of $2.8 \sigma$. It should be noted, however, that there is a large statistical probability of such an excess to appear somewhere in the parameter space under study.

1. In the no mixing benchmark set the stop mixing parameter $X_{\mathrm{t}}$ is put at zero. The other parameters are fixed at the following values: $m_{\mathrm{SUSY}}=1 \mathrm{TeV}, M_{2}=200 \mathrm{GeV}$, $\mu=-200 \mathrm{GeV}$. The gluino mass $m_{\tilde{\mathrm{g}}}$ has little effect on the phenomenology of this scenario; its value is set to $800 \mathrm{GeV}$.

The corresponding exclusion plots are shown in Fig. 14, The unexcluded region with $64<m_{\mathrm{h}}<88 \mathrm{GeV}$ and $m_{\mathrm{A}}<43 \mathrm{GeV}$ is due to the dominance of the cascade decay $h \rightarrow$ AA for which the search sensitivity is lower than for the $h \rightarrow b \bar{b}$ and $\tau^{+} \tau^{-}$ channels. One should note, however, that in this domain the charged Higgs boson mass $m_{\mathrm{H}^{ \pm}}$is predicted to smaller than $81 \mathrm{GeV}$. This area is probed by charged Higgs boson searches [53], which will be further extended in the future (see Fig. 14 (d)). 
The region with $m_{\mathrm{h}}>83 \mathrm{GeV}$ and $m_{\mathrm{A}}>82 \mathrm{GeV}$ is still unexcluded. In this domain, either the cross-section for Higgsstrahlung $\mathrm{e}^{+} \mathrm{e}^{-} \rightarrow \mathrm{hZ}$ is $\operatorname{small}\left(\sin ^{2}(\beta-\alpha)\right.$ is close to 0, see Eq. 1 in Section 3) or the pair production process $\mathrm{e}^{+} \mathrm{e}^{-} \rightarrow \mathrm{hA}$ is kinematically forbidden.

Values of $\tan \beta$ are excluded from 0.8 to 6.2 . However, the $\tan \beta$ limit is strongly dependent on the top quark mass which was taken to be $m_{\text {top }}=174.3 \mathrm{GeV}$. For $m_{\text {top }}=179 \mathrm{GeV}$, the $\tan \beta$ exclusion is reduced to $0.8<\tan \beta<4.7$. If one disregards the unexcluded domain at low $\tan \beta$, the following lower bounds are obtained at the $95 \%$ confidence level: $m_{\mathrm{h}}>83 \mathrm{GeV}$ and $m_{\mathrm{A}}>82 \mathrm{GeV}$.

The constraint from the measured value of $\mathrm{BR}(\mathrm{b} \rightarrow \mathrm{s} \gamma)$ (see Section 5.2) is indicated in Fig. 14 (b).

2. The no mixing ( $2 \mathrm{TeV}$ ) benchmark set differs from the no mixing scenario in the flipped sign of $\mu$ (which is preferred by the current results on $\left.(g-2)_{\mu}\right)$ and by a larger SUSY mass scale $M_{S U S Y}=2 \mathrm{TeV}$. The value of $\tan \beta$ is scanned only from 0.7 to 40 due to numerical instabilities in the diagonalisation of the mass matrix for very low $\tan \beta$. Therefore the largest part of the unexcluded region of the no mixing case at low $\tan \beta$ is not probed in this scenario.

The corresponding exclusion region is shown in Fig. 15. For $m_{\mathrm{A}}>2 \mathrm{GeV}$, i.e. above the region of resonant Higgs boson decays, absolute limits can be set for the Higgs boson masses and on $\tan \beta$, which are $m_{\mathrm{h}}>83.3 \mathrm{GeV}, m_{\mathrm{A}}>84.3 \mathrm{GeV}$ and $\tan \beta>4$.2. If the unexcluded area at $m_{\mathrm{A}}<2 \mathrm{GeV}$ is also regarded, the exclusion in $\tan \beta$ is $0.9<\tan \beta<4.2$. The reduced $\tan \beta$ exclusion with respect to the no mixing case reflects the increased value of $M_{S U S Y}$. This limit is further weakened to $\tan \beta>3.2$ for $m_{\text {top }}=179 \mathrm{GeV}$.

The measurements of $\mathrm{BR}(\mathrm{b} \rightarrow \mathrm{s} \gamma)$ exclude the no mixing $(2 \mathrm{TeV})$ scenario for $m_{\mathrm{A}}<$ $450 \mathrm{GeV}$, as can be seen in Fig. 15] (b).

3. The $m_{\mathrm{h}}-\max$ benchmark set is designed to yield the largest range of $m_{\mathrm{h}}$ for a given $\left(m_{\mathrm{A}}, \tan \beta\right)$. This scenario is therefore the most conservative in terms of exclusion in $\tan \beta$. The other parameters are fixed as in the no mixing scenario, with the exception of the stop mixing parameter $X_{t}=\sqrt{6} \mathrm{TeV}$.

The exclusion plots for this benchmark set are shown in Fig. 16. The following absolute limits are obtained at the $95 \%$ confidence level: $m_{\mathrm{h}}>84.5 \mathrm{GeV}$ and $m_{\mathrm{A}}>85.0 \mathrm{GeV}$. Furthermore, values of $\tan \beta$ between 0.7 and 1.9 are excluded. For $m_{\text {top }}=179 \mathrm{GeV}$ this exclusion shrinks to the domain $1.0<\tan \beta<1.3$. Since the $m_{\mathrm{h}}-\max$ benchmark set yields the most conservative exclusion in $\tan \beta$, also $m_{\text {top }}=183 \mathrm{GeV}$ as the anticipated $1 \sigma$ upper bound of an increased world average of $m_{\text {top }}=179 \mathrm{GeV}$ was tested. This is illustrated in Fig[16 (b) and in Fig[16 (c), where the exclusion in the $\left(\tan \beta, m_{\mathrm{A}}\right)$ plane respectively the theoretical upper bounds on $m_{\mathrm{h}}$ for $m_{\mathrm{top}}=179 \mathrm{GeV}$ and $m_{\mathrm{top}}=183 \mathrm{GeV}$ are also shown. Should the world average of the top quark mass move beyond $179.5 \mathrm{GeV}$, the exclusion in $\tan \beta$ would vanish completely.

The supplementary constraint from the measured value of the $B R(b \rightarrow s \gamma)$ is shown in Fig. [16 (b). 
4. The $m_{\mathrm{h}}-\max ^{+}$benchmark set differs from the $m_{\mathrm{h}}-\max$ case only by the flipped sign of $\mu$. This choice is favored by the presently available results on $(g-2)_{\mu}[54$. Since the Higgs boson properties depend only weakly on the sign of $\mu$, the accessible Higgs mass range as well as the excluded domains are very similar to those of the $m_{\mathrm{h}}-$ max scenario; they are shown in Fig. 17.

The limits on the Higgs masses are $m_{\mathrm{h}}>84.5 \mathrm{GeV}$ and $m_{\mathrm{A}}>84.0 \mathrm{GeV}$. The excluded range in $\tan \beta$ is $0.7<\tan \beta<1.9$, which decreases to $0.96<\tan \beta<1.4$ for $m_{\mathrm{top}}=179 \mathrm{GeV}$.

The $m_{\mathrm{h}}-\max ^{+}$scenario is excluded for $m_{\mathrm{A}}<600 \mathrm{GeV}$ by $\mathrm{BR}(\mathrm{b} \rightarrow \mathrm{s} \gamma)$ measurements for all values of $\tan \beta$ considered (between 0.4 and 40 ). This means that only the decoupling limit with $m_{\mathrm{h}}$ at its maximum value for a given $\tan \beta$ is still allowed by $\mathrm{BR}(\mathrm{b} \rightarrow \mathrm{s} \gamma)$.

5. The constrained $m_{h}$-max benchmark set differs from the $m_{\mathrm{h}}-\max ^{+}$set by the flipped sign of $X_{\mathrm{t}}$, which yields better agreement with $\mathrm{BR}(\mathrm{b} \rightarrow \mathrm{s} \gamma)$ constraints. One observes that the maximum value of the Higgs boson mass at a given $\tan \beta$ is lowered by about $5 \mathrm{GeV}$.

The excluded areas for this scenario (see Fig. 18) show similar features as the $m_{\mathrm{h}}-\max$ and $m_{\mathrm{h}}-\max ^{+}$scenarios. The limits on the Higgs masses are $m_{\mathrm{h}}>$ $84.0 \mathrm{GeV}$ and $m_{\mathrm{A}}>85.0 \mathrm{GeV}$. The excluded range in $\tan \beta$ is $0.6<\tan \beta<2.2$, which shrinks to $0.8<\tan \beta<1.8$ for $m_{\text {top }}=179 \mathrm{GeV}$. This is illustrated in Fig. 18) (b) and (c).

The supplementary constraint from the measured value of the $\mathrm{b} \rightarrow \mathrm{s} \gamma$ branching ratio is shown in Fig. 18 (b) as the band delimited by the two dash-dotted lines.

6. The large $\mu$ benchmark set is designed to illustrate choices of parameters for which the detection of the Higgs bosons is believed to be a priori difficult at LEP. The parameters are set to the following values: $m_{\mathrm{SUSY}}=400 \mathrm{GeV}, \mu=1 \mathrm{TeV}, M_{2}=$ $400 \mathrm{GeV}, m_{\tilde{\mathrm{g}}}=200 \mathrm{GeV}, X_{t}=-300 \mathrm{GeV}$. It is scanned from $\tan \beta=0.7-40$ and $m_{\mathrm{A}}=0-400$.

For this set of parameters, the $\mathrm{h}$ boson is always kinematically accessible $\left(m_{\mathrm{h}}<\right.$ $108 \mathrm{GeV}$ ) but its decay to b $\bar{b}$, on which most of the searches are based, is suppressed. For many of the scan points the decay $h \rightarrow \tau^{+} \tau^{-}$is also suppressed. The dominant decay modes are thus $\mathrm{h} \rightarrow \mathrm{c} \overline{\mathrm{c}}$, gg or $\mathrm{W}^{+} \mathrm{W}^{-}$, and the detection of Higgs bosons has to rely more heavily on flavour-independent searches.

In some of the scan points the Higgsstrahlung process $\mathrm{e}^{+} \mathrm{e}^{-} \rightarrow \mathrm{hZ}$ is suppressed alltogether $\left(\sin ^{2}(\beta-\alpha)\right.$ small). However, the heavy neutral scalar is relatively light in such cases $\left(m_{\mathrm{H}}<109 \mathrm{GeV}\right)$ and the cross-section for the process $\mathrm{e}^{+} \mathrm{e}^{-} \rightarrow \mathrm{HZ}$, being proportional to $\cos ^{2}(\beta-\alpha)$, is large.

The exclusions for this benchmark scenario are given in Fig. 19, They show that the parameter space is essentially excluded even in this difficult scenario, with the exception of a few isolated "islands". Those may slightly increase for higher values of the top quark mass. The origin of the islands can best be explained using Fig. 19](b). The large diagonal island at $m_{\mathrm{A}}>100 \mathrm{GeV}$ is due to the fact that $\mathrm{BR}(\mathrm{h} \rightarrow \mathrm{b} \overline{\mathrm{b}})$ goes to 0 there. The two thin vertical islands around $m_{\mathrm{A}}>100 \mathrm{GeV}$ are due to an overlap between $\mathrm{e}^{+} \mathrm{e}^{-} \rightarrow \mathrm{hZ}$ and $\mathrm{e}^{+} \mathrm{e}^{-} \rightarrow \mathrm{HZ}$ production. Both are kinematically accessible, but either one or the other can be used in the interpretation. 
The supplementary constraint from the measured value of the $\mathrm{b} \rightarrow \mathrm{s} \gamma$ branching ratio is shown in Fig. 19 (b).

7. The gluophobic benchmark set is constructed such that the Higgs coupling to gluons is suppressed due to a cancellation between the top and the stop loops at the hgg vertex. Since at the LHC the searches will rely heavily on the production of the Higgs boson by gluon-gluon fusion, such a scenario may be difficult to investigate there. The parameters chosen are $: m_{\mathrm{SUSY}}=350 \mathrm{GeV}, M_{2}=300 \mathrm{GeV}, \mu=300 \mathrm{GeV}$, $X_{t}=-750 \mathrm{GeV}, 0.4<\tan \beta<40,0 \mathrm{GeV}<m_{\mathrm{A}}<1 \mathrm{TeV}$ and $m_{\tilde{\mathrm{g}}}=500 \mathrm{GeV}$.

The exclusion for this benchmark set is shown in Fig. 20, It is excluded to a large extend. The limits on the Higgs masses are $m_{\mathrm{h}}>82 \mathrm{GeV}$ and $m_{\mathrm{A}}>87.5 \mathrm{GeV}$. The excluded range in $\tan \beta$ is $\tan \beta<6.0$. The excluded range is reduced to $\tan \beta<3.5$ for $m_{\mathrm{top}}=179 \mathrm{GeV}$.

The supplementary constraint from the measured value of the $\mathrm{b} \rightarrow \mathrm{s} \gamma$ branching ratio is shown in Fig. 20 (b).

8. In the small $\alpha_{\text {eff }}$ benchmark set the Higgs boson decay channels $\mathrm{h} \rightarrow \mathrm{b} \overline{\mathrm{b}}$ and $\mathrm{h} \rightarrow$ $\tau^{+} \tau^{-}$are suppressed with respect to their Standard Model coupling by the additional factor $-\sin \alpha_{\text {eff }} / \cos \beta$, stemming from corrections from $\tilde{b}-\tilde{g}$ loops. This scenario may also be difficult to investigate by the LHC experiments. Similarly to the large$\mu$ scenario, such suppressions occur for large $\tan \beta$ and not too large $m_{A}$. The parameters chosen are: $m_{\mathrm{SUSY}}=800 \mathrm{GeV}, M_{2}=500 \mathrm{GeV}, \mu=2 \mathrm{TeV}, X_{t}=$ $-1100 \mathrm{GeV}, 0.4<\tan \beta<40$, and $m_{\tilde{\mathrm{g}}}=500 \mathrm{GeV}$.

The exclusion for this benchmark set is shown in Fig. 21] The limits on the Higgs masses are $m_{\mathrm{h}}>79.0 \mathrm{GeV}$ and $m_{\mathrm{A}}>90.0 \mathrm{GeV}$. The excluded range in $\tan \beta$ is $0.4<\tan \beta<3.6$, which is reduced to $0.5<\tan \beta<2.9$ for $m_{\text {top }}=179 \mathrm{GeV}$. It appears that effects of suppression of the decays $h \rightarrow b \bar{b}$ and $h \rightarrow \tau^{+} \tau^{-}$do not play a role in the region kinematically accessible at LEP.

The constraint from the measured value of $\mathrm{BR}(\mathrm{b} \rightarrow \mathrm{s} \gamma)$ is shown in Fig. 21 (b).

\subsection{CPV benchmark scenarios}

In the MSSM, the Higgs potential is invariant under CP transformations at tree level. However it is possible to explicitly or spontaneously break $\mathrm{CP}$ symmetry by radiative corrections [55]. In particular, the phases of $A_{\mathrm{t}, \mathrm{b}}$ and $m_{\tilde{\mathrm{g}}}$ introduce CP violation into the Higgs potential via loop effects, leading to sizeable off-diagonal contributions to the Higgs boson mass matrix (see Section 31).

As a consequence the Higgs mass eigenstates $\mathrm{H}_{1}, \mathrm{H}_{2}$ and $\mathrm{H}_{3}$ are not the CP eigenstates. This influences predominantly the couplings in the Higgs sector. Fig. 22 shows the coupling of a mixed mass eigenstate $\mathrm{H}_{1}$ consisting of admixtures from the $\mathrm{CP}$ eigenstates h, H and A. Since only the CP-even field component couples to the $\mathrm{Z}$ boson, the individual coupling of the mass eigenstates are reduced in the CPV case with respect to a CPC case.

The size of the CPV off-diagonal elements, $\mathcal{M}_{i j}^{2}$, of the Higgs boson mass matrix and hence the size of CPV effect scales qualitatively [28] as

$$
\mathcal{M}_{i j}^{2} \propto \frac{m_{\mathrm{top}}^{4}}{v^{2}} \frac{\operatorname{Im}\left(\mu A_{t}\right)}{32 \pi^{2} m_{\mathrm{SUSY}}^{2}} .
$$


Large CPV effects, and thus scenarios dissimilar from the CPC case, are therefore obtained if the SUSY breaking scale $m_{\mathrm{SUSY}}$ is small and the imaginary contribution to $\mu A_{t}$ large. Also large values of $m_{\text {top }}$ increase the CPV effects.

When choosing the parameters, experimental constraints [56, 57] from electric dipole moment (EDM) measurements of the neutron and the electron have to be fulfilled. However, cancellations among different contributions to the EDM may naturally emerge [55] hence those measurements provide no universal exclusion in the MSSM parameter space, while direct searches at LEP provide a good testing ground for a CPV MSSM.

The basic CPV MSSM benchmark set is CPX. Its parameters are chosen such as to approximately fulfill the EDM constraints and to provide features that are the most dissimilar from a CPC scenario. The choice of parameters [28] is given in Table 14] (last column). In the definition of the CPX scenario [28] the relations $\mu=4 m_{\mathrm{SUSY}}$ and $\left|A_{\mathrm{t}, \mathrm{b}}\right|=\left|m_{\tilde{\mathrm{g}}}\right|=2 m_{\mathrm{SUSY}}$ are fixed. Here, $m_{\mathrm{SUSY}}=500 \mathrm{GeV}$ is chosen. The parameter $m_{2}$ is set to $200 \mathrm{GeV}$. Additionally the complex phases of $A_{\mathrm{t}, \mathrm{b}}$ and $m_{\tilde{g}}$ are fixed at $90^{\circ}$ degrees. Variants of the CPX scenario are investigated to check the stability of the CPX results with respect to the choice of its parameters. The phases of $A_{\mathrm{t}, \mathrm{b}}$ and $m_{\tilde{g}}$ varied from from $0^{\circ}$ to $180^{\circ}, \mu$ in between 500 and $4000 \mathrm{GeV}$. The scenario with $\arg \left(A_{\mathrm{t}, \mathrm{b}}\right)=90^{\circ}$ has very different features from a CPC case and therefore has good properties for a $\mathrm{CPV}$ benchmark scenario.

The benchmark scan databases, containing masses, cross-sections and branching ratios for all three neutral Higgs bosons for a variety of different input parameters, are generated using both CPH [28, a modified version of SUBHPOLE, and FEYNHIGGS 2.0 46. They are implemented in a modified version of HZHA [19]. Initial-state radiation and interference between Higgsstrahlung and boson fusion processes are taken into account by HZHA. The parameter $\tan \beta$ is scanned from 0.6 to 40 , and $m_{\mathrm{H}^{ \pm}}$is scanned from 4 to 1000. In this region both $\mathrm{H}_{1}$ and $\mathrm{H}_{2}$ have a width below $1 \mathrm{GeV}$, negligible with respect to the experimental resolution of several $\mathrm{GeV}$.

Also in the CPV scenarios there is good agreement between the data and the background estimation. The largest observed excess of the data over the background appears at $m_{\mathrm{H}_{1}}=40 \mathrm{GeV}$ and $m_{\mathrm{H}_{2}}=105 \mathrm{GeV}$ in the CPX benchmark set. The excess has $\left(1-\mathrm{CL}_{b}\right)=1.2 \times 10^{-3}$, corresponding to a significance of $3.0 \sigma$. Also here it should be noted that there is a large statistical probability of such an excess to appear somewhere in the parameter space under study. Limits on the MSSM parameter space are derived.

Fig. 23 shows the combined exclusion result for the CPX scenario with all phases equal to $90^{\circ}, m_{\mathrm{SUSY}}=500 \mathrm{GeV}$ and $\mu=2 \mathrm{TeV}$. Fig. 23 (a) shows both the expected and observed 95\% CL exclusion areas in the plane of $m_{\mathrm{H}_{1}}$ and $m_{\mathrm{H}_{2}}$. For heavy $m_{\mathrm{H}_{2}}$, $\mathrm{H}_{1}$ resembles the $\mathrm{SM}$ Higgs boson (almost completely CP-even) with very little effect from $\mathrm{CP}$ violation. The limit on the allowed mass of $\mathrm{H}_{1}$ for large $m_{\mathrm{H}_{2}}$ is found to be $m_{\mathrm{H}_{1}}>112 \mathrm{GeV}$. In the region below $m_{\mathrm{H}_{2}} \approx 130 \mathrm{GeV}$ CPV effects play a major role.

Fig. 23] (b) shows the 95\% CL exclusion areas in the parameter space of $\tan \beta$ and $m_{\mathrm{H}_{2}}$. One can see that $\tan \beta<2.8$ is excluded. The band at $\tan \beta<2.8$ is excluded by searches for the SM-like $\mathrm{H}_{1}$, while the band at $\tan \beta>10$ and $m_{\mathrm{H}_{2}}<120 \mathrm{GeV}$ is excluded by searches for $\mathrm{ZH}_{2}$ and $\mathrm{H}_{1} \mathrm{H}_{2}$ topologies.

Fig. 23 (c) displays the parameter space of $\tan \beta$ and $m_{\mathrm{H}_{1}}$. The range $\tan \beta<2.8$ is excluded, and a lower limit of $\tan \beta>3.2$ exists if $m_{\mathrm{H}_{1}}$ is below $112 \mathrm{GeV}$. For $4<\tan \beta<$ $10, \mathrm{ZH}_{2}$ production is dominant. The large difference between the expected and observed exclusion regions in the area of $4<\tan \beta<10$ is mainly due to a less than $2 \sigma$ excess in the data between $m_{\mathrm{h}} \approx 95 \mathrm{GeV}$ and $m_{\mathrm{h}} \approx 110 \mathrm{GeV}[7$, which corresponds to the mass of 
$\mathrm{H}_{2}$ in this region. For $m_{\mathrm{H}_{1}}<50 \mathrm{GeV}$ there are also unexcluded regions in the expected exclusion, which is due to dominant $\mathrm{ZH}_{2} \rightarrow \mathrm{ZH}_{1} \mathrm{H}_{1}$ production with relatively large $m_{\mathrm{H}_{1}}$, yielding broad mass resolutions and therefore reduced sensitivity.

In Fig. 23 (d) the exclusion area is shown in the parameter space of the theoretical input parameters $\tan \beta$ and $m_{\mathrm{H}^{ \pm}}$, which are varied during the scan. Since the CPX scenario yields $m_{\mathrm{H}_{2}} \approx m_{\mathrm{H}^{ \pm}}$for most of the scan points, this is very similar to Fig. 23 (b).

The uncertainty inherent to the two theoretical approaches, CPH and FEYNHIGGS, is illustrated in parts (e) and (f) of Fig. 23. The largest discrepancy occurs for large values of $\tan \beta$, where the FEYNHIGGS calculation (part (f)) predicts a higher cross-section for Higgsstrahlung, and hence a better search sensitivity than the CPH prediction (part(e)).

The large impact of the value of the top quark mass on the exclusion limits is shown in Fig. 24. For $m_{\text {top }}=179.3 \mathrm{GeV}$, the excluded range in $\tan \beta$ shrinks to $\tan \beta<2.4$.

The effect of different choices of the CPV phases is illustrated in Figs. 25 and 26. Values of $\arg \left(A_{\mathrm{t}, \mathrm{b}}\right)=\arg \left(m_{\tilde{\mathrm{g}}}\right)$ from $0^{\circ}$ to $180^{\circ}$ are displayed. Fig. 25] shows exclusion regions in the parameter space of $\tan \beta$ and $m_{\mathrm{H}_{1}}$ for $\arg \left(A_{\mathrm{t}, \mathrm{b}}\right)=\arg \left(m_{\tilde{\mathrm{g}}}\right)=90^{\circ}, 60^{\circ}, 30^{\circ}$ and $0^{\circ}$. The lower limit on $\tan \beta>1.9$ in the scenario with phases of $60^{\circ}$ is the lowest limit on $\tan \beta$ in the CPV scenarios. At $30^{\circ}$ and at $0^{\circ}$ all areas for low $m_{\mathrm{H}_{1}}$ and low $\tan \beta$ are excluded. The exclusion for the maximally CPV scenario CPX with $90^{\circ}$ is very different from the exclusion of a CPC scenario $\left(\arg \left(A_{\mathrm{t}, \mathrm{b}}\right)=\arg \left(m_{\tilde{\mathrm{g}}}\right)=0^{\circ}\right)$. A variation of the second main parameter governing the size of CPV effects, $m_{\mathrm{SUSY}}$, has similar effects on the exclusion to those of a variation of $\arg \left(A_{\mathrm{t}, \mathrm{b}}\right)=\arg \left(m_{\tilde{\mathrm{g}}}\right)$.

Fig. 26] shows exclusion regions in the parameter space of $\tan \beta$ and $m_{\mathrm{H}_{1}}$ for phases of (a) $135^{\circ}$ and (b) $180^{\circ}$. The scenario in (a) is phenomenologically still similar to the original CPX scenario. The scenario in (b), which is in fact a CPC case, exhibits two allowed regions, of which the lower one from $\tan \beta=3$ to $\tan \beta=13$ has a low $\mathrm{H}_{1} \mathrm{Z}$ coupling. The unexcluded "hole" in the exclusion region for $90<m_{\mathrm{H}_{1}}<100 \mathrm{GeV}$ is due to an excess of the background in the SM-like channels.

Since the CPX scenario has a relatively high value of $\mu=2 \mathrm{TeV}$, which influences the mixing of the $\mathrm{CP}$ eigenstates into the mass eigenstates (see Eq. (6) ),$\mu$ is varied from $\mu=500 \mathrm{GeV}$ to $\mu=4 \mathrm{TeV}$ in Fig. 27. For $\mu=500 \mathrm{GeV}$ (Fig. 27 (a)) and $\mu=1 \mathrm{TeV}$ (Fig. 27 (b)) the CPV effects are small. Therefore no unexcluded regions occur at small $m_{\mathrm{H}_{1}}$. The scenario with $\mu=4 \mathrm{TeV}$ (Fig. 27(d)) has strong mixing and a suppression of pair production at large $\tan \beta$, resulting in an exclusion area that is considerably smaller than in the CPX scenario (Fig. 27 (c)).

The proposal of the CPX scenario in [28] leaves the choice of $m_{\mathrm{SUSY}}$ open, as long as the relations $\left|A_{\mathrm{t}, \mathrm{b}}\right|=2 m_{\mathrm{SUSY}},\left|m_{\tilde{\mathrm{g}}}\right|=2 m_{\mathrm{SUSY}}$ and $\mu=4 m_{\mathrm{SUSY}}$ are preserved. In order to test the dependence on $m_{\mathrm{SuSY}}$, two scenarios are tested: Fig. 28 (a) shows the scenario $\mathrm{CPX}_{1.0}$, where the ratio between the parameters in the CPX proposal is preserved, while $m_{\mathrm{SUSY}}$ is increased from $500 \mathrm{GeV}$ to $1 \mathrm{TeV}$. Only small differences with respect to the CPX scenario with $m_{\mathrm{SUSY}}=500 \mathrm{GeV}$ can be seen. Fig. 28 (b) shows the CPX scenario as given in Table 14, but with only $m_{\mathrm{SUSY}}$ set to $1 \mathrm{TeV}$, while the values of $\left|A_{\mathrm{t}, \mathrm{b}}\right|,\left|m_{\tilde{\mathrm{g}}}\right|$ and $\mu$ are kept fixed. This results in a decrease of the CPV effects and thus no unexcluded regions at small $m_{\mathrm{H}_{1}}$ are observed. 


\section{Summary}

The searches for neutral Higgs bosons described in this paper are based on all data collected by the OPAL experiment, at energies in the vicinity of the $\mathrm{Z}$ resonance (LEP1 phase) and between 130 and $209 \mathrm{GeV}$ (LEP2 phase). The corresponding integrated luminosities are of about $720 \mathrm{pb}^{-1}$. The searches addressing the Higgsstrahlung process $\mathrm{e}^{+} \mathrm{e}^{-} \rightarrow \mathcal{H} Z$ and those for the pair production process $\mathrm{e}^{+} \mathrm{e}^{-} \rightarrow \mathcal{H}_{1} \mathcal{H}_{2}$ are statistically combined. None of these searches reveals a significant excess of events beyond the predicted background level, which would indicate the production of Higgs bosons.

From these results, model-independent limits are derived for the cross-section of a number of event topologies that could be associated to Higgs boson production. These limits cover a wide range of Higgs boson masses and are typically much lower than the largest cross-sections predicted by the MSSM.

The search results are also used to test a number of "benchmark scenarios" of the MSSM, with and without the inclusion of CP-violating effects.

In the CP-conserving case, new benchmark situations are investigated as compared to earlier publications. These are motivated either by new measurements of the $\mathrm{b} \rightarrow \mathrm{s} \gamma$ branching ratio and the muon anomalous magnetic moment $(g-2)_{\mu}$, or in anticipation of the forthcoming searches at the proton-proton collider LHC. In all these scenarios the searches conducted by OPAL exclude sizeable domains of the MSSM parameter space, even in those situations where the sensitivity of the LHC experiments is expected to be low. An overview of the results is given in Table 15, In the " $m_{\mathrm{h}}$-max" scenario which, among all scenarios predicts the widest range of $m_{\mathrm{h}}$ values, the following limits can be set at the $95 \%$ confidence level: $m_{\mathrm{h}}>84.5 \mathrm{GeV}$ and $m_{\mathrm{A}}>85.0 \mathrm{GeV}$; furthermore, if the top quark mass is fixed at the current experimental value of $174.3 \mathrm{GeV}$, the range $0.7<\tan \beta<1.9 \mathrm{GeV}$ can be excluded (this range shrinks for higher values of $m_{\mathrm{top}}$ ).

For the first time, a number of CP-violating MSSM scenarios are studied experimentally, where the CP-violating effects are introduced in the Higgs potential by radiative corrections. The "CPX" benchmark scenario is designed to maximize the phenomenological differences in the Higgs sector with respect to the CP-conserving scenarios. In this case the region $\tan \beta<2.8$ is excluded at $95 \%$ confidence level but no universal limit is obtained for either of the Higgs boson masses. However, for $\tan \beta<3.3$, the limit $m_{\mathrm{H}_{1}}>112 \mathrm{GeV}$ can be set for the mass of the lightest neutral Higgs boson of the model.

\section{Acknowledgments}

We particularly wish to thank Marcela Carena, Sven Heinemeyer, Gudrun Hiller, Steve Mrenna, Apostolos Pilaftsis and Georg Weiglein for their support. We also thank the SL Division for the efficient operation of the LEP accelerator at all energies and for their close cooperation with our experimental group. In addition to the support staff at our own institutions we are pleased to acknowledge the

Department of Energy, USA, National Science Foundation, USA, Particle Physics and Astronomy Research Council, UK, Natural Sciences and Engineering Research Council, Canada, Israel Science Foundation, administered by the Israel Academy of Science and Humanities, 
Benoziyo Center for High Energy Physics,

Japanese Ministry of Education, Culture, Sports, Science and Technology (MEXT) and a grant under the MEXT International Science Research Program,

Japanese Society for the Promotion of Science (JSPS),

German Israeli Bi-national Science Foundation (GIF),

Bundesministerium für Bildung und Forschung, Germany,

National Research Council of Canada,

Hungarian Foundation for Scientific Research, OTKA T-038240, and T-042864,

The NWO/NATO Fund for Scientific Research, the Netherlands.

\section{References}

[1] P. W. Higgs, Phys. Lett. 12 (1964) 132;

F. Englert and R. Brout, Phys. Rev. Lett. 13 (1964) 321.

[2] R. Barate et al. [ALEPH, DELPHI, L3, OPAL Collaborations], Phys. Lett. B 565 (2003) 61.

[3] A. Brignole, G. Degrassi, P. Slavich and F. Zwirner, Nucl. Phys. B 631 (2002) 195;

G. Degrassi, S. Heinemeyer, W. Hollik, P. Slavich and G. Weiglein, Eur. Phys. J. C 28 (2003) 133.

[4] A. Pilaftsis and C. E. Wagner, Nucl. Phys. B 553 (1999) 3.

[5] M. Carena et al., Nucl. Phys. B 599 (2001) 158.

[6] S. Heinemeyer and W. Hollik, Nucl. Phys. B 474 (1996) 32.

[7] G.Abbiendi et al. [OPAL Collaboration], Eur. Phys. J. C 26 (2003) 479.

[8] G. Abbiendi et. al. [OPAL Collaboration], Eur. Phys. J. C 12 (2000) 567.

[9] G. Abbiendi et. al. [OPAL Collaboration], Eur. Phys. J. C 7 (1999) 407.

[10] K. Ackerstaff et. al. [OPAL Collaboration], Eur. Phys. J. C 1 (1998) 425.

[11] K. Ackerstaff et. al. [OPAL Collaboration], Eur. Phys. J. C 5 (1998) 19.

[12] G. Alexander et. al. [OPAL Collaboration], Z. Phys. C 73 (1997) 189.

[13] R. Akers et. al. [OPAL Collaboration], Z. Phys. C 64 (1994) 1.

[14] A. Heister et al. [ALEPH Collaboration], Phys. Lett. B 526 (2002) 191.

J. Abdallah et al. [DELPHI Collaboration], Eur. Phys. J. C 32 (2004) 145; P. Achard et al. [L3 Collaboration], Phys. Lett. B 545 (2002) 30.

[15] T. Affolder et al. [CDF Collaboration], Phys. Rev. Lett. 86 (2001) 4472.

[16] M. Carena, S. Heinemeyer, C. E. M. Wagner and G. Weiglein, hep-ph/0202167.

[17] K. Ahmet et al. [OPAL Collaboration], Nucl. Instr. and Meth. A 305 (1991) 275. 
[18] S. Anderson et al., Nucl. Instr. and Meth. A 403 (1998) 326.

[19] P. Janot, Physics at LEP2, CERN 96-01 Vol. 2309.

[20] S. Jadach, B. F. Ward and Z. Wás, Comput. Phys. Commun. 130 (2000) 260.

[21] J. Fujimoto et al., Comp. Phys. Comm. 100 (1997) 128;

J. Fujimoto et al., Physics at LEP2, CERN 96-01, Vol.2, 30.

[22] S. Jadach, W. Płaczek, and B.F.L. Ward, Physics at LEP2, CERN 96-01, Vol.2, 286;

Phys. Lett. B 390 (1997), 298.

[23] E. Budinov et al., Physics at LEP2, CERN 96-01, Vol.2, 216;

R. Engel and J. Ranft, Phys. Rev. D 54 (1996) 4244.

[24] G. Marchesini et al., Comp. Phys. Comm. 67 (1992) 465; G. Corcella et al., JHEP 0101 (2001) 10.

[25] J.A.M. Vermaseren, Nucl. Phys. B 229 (1983) 347.

[26] T. Sjöstrand, Comp. Phys. Comm. 82 (1994) 74;

T. Sjöstrand, LU TP 95-20.

[27] J. Allison et al., Nucl. Instr. and Meth. A 317 (1992) 47.

[28] M. Carena, J. R. Ellis, A. Pilaftsis and C. E. Wagner, Phys. Lett. B 495 (2000) 155.

[29] G. Abbiendi et al. [OPAL Collaboration], Eur. Phys. J. C27 (2003) 483.

[30] G. Abbiendi et al. [OPAL Collaboration], Eur. Phys. J. C 18 (2001) 425.

[31] G. Abbiendi et al. [OPAL Collaboration], hep-ex/0312042, Submitted to Phys. Lett. B.

[32] N. Brown and W. J. Stirling, Phys. Lett. B 252 (1990) 657.

[33] K. Ackerstaff et al. [OPAL Collaboration], Eur. Phys. J. C 2 (1998) 441.

[34] G. Parisi, Phys. Lett. B 74 (1978) 65.

J. F. Donoghue, F. E. Low and S. Y. Pi, Phys. Rev. D 20 (1979) 2759.

[35] K. Ackerstaff et al. [OPAL Collaboration], Eur. Phys. J. C 2 (1998) 213.

[36] The LEP Collaborations ALEPH, DELPHI, L3, OPAL, the LEP Electroweak Working Group, and the SLD Heavy Flavour and Electroweak Groups, hep-ex/0212036.

[37] D. Y. Bardin, P. Christova, M. Jack, L. Kalinovskaya, A. Olchevski, S. Riemann and T. Riemann, Comput. Phys. Commun. 133 (2001) 229.

[38] G.Abbiendi et al. [OPAL Collaboration], Eur. Phys. J. C 27 (2002) 311.

[39] G. Abbiendi et al. [OPAL Collaboration], Eur. Phys. J. C 23 (2002) 397. 
[40] P. Cho, M. Misiak and D. Wyler, Phys. Rev. D 54, 3329 (1996);

A. Kagan and M. Neubert, Eur. Phys. J. C 7 (1999) 5;

K. Chetyrkin, M. Misiak and M. Munz, Phys. Lett. B 400, (1997) 206, [Erratumibid. 425 (1998) 414];

P. Gambino and M. Misiak, Nucl. Phys. B 611 (2001) 338;

J. R. Ellis, T. Falk, G. Ganis, K. A. Olive and M. Srednicki, Phys. Lett. B 510 (2001) 236.

[41] R. Barate et al. [ALEPH Collaboration], Phys. Lett. B 429 (1998) 169;

S. Chen et al. [CLEO Collaboration], Phys. Rev. Lett. 87 (2001) 251807;

K. Abe et al. [Belle Collaboration], Phys. Lett. B 511 (2001) 151;

B. Aubert et al. [BABAR Collaboration], hep-ex/0207074; hep-ex/0207076.

[42] F. A. Berends and R. Kleiss, Nucl. Phys. B 260 (1985) 32.

[43] D.E. Groom et al, Eur. Phys. J. C 15 (2000) 1, available on the PDG WWW pages http://pdg.lbl.gov/

[44] M. Carena, S. Heinemeyer, C. E. M. Wagner and G. Weiglein, hep-ph/9912223.

[45] S. Heinemeyer, W. Hollik and G. Weiglein, Comp. Phys. Comm. 124 (2000) 76; Also see http://www.feynhiggs.de.

[46] M. Frank, S. Heinemeyer, W. Hollik and G. Weiglein, hep-ph/0212037 Also see http://www.feynhiggs.de.

[47] S. Heinemeyer, W. Hollik and G. Weiglein, Eur. Phys. Jour. C 9 (1999) 343.

[48] S. Heinemeyer, W. Hollik and G. Weiglein, Phys. Rev. D 58 (1998) 091701, Phys. Lett. B 440 (1998) 296, hep-ph/9807423 and JHEP 0006 (2000) 009.

[49] M. Carena, M. Quirós and C.E.M. Wagner, Nucl. Phys. B 461 (1996) 407.

[50] M. Carena, S. Mrenna and C. Wagner, Phys. Rev. D 60 (1999) 075010.

[51] M. Carena, H. E. Haber, S. Heinemeyer, W. Hollik, C. E. M. Wagner and G. Weiglein, Nucl. Phys. B 580 (2000) 29.

[52] J. R. Espinosa and R.-J. Zhang, JHEP 0003 (2000) 026.

[53] P. Bock et al. [ALEPH, DELPHI, L3 and OPAL Collaborations], CERN-EP-2000-055

[54] G. W. Bennett et al. [Muon g-2 Collaboration], Phys. Rev. Lett. 89 (2002) 101804 [Erratum-ibid. 89 (2002) 129903].

[55] M. Carena, J. R. Ellis, A. Pilaftsis and C. E. Wagner, Nucl. Phys. B 586 (2000) 92.

[56] E. D. Commins, S. B. Ross, D. DeMille and B. C. Regan, Phys. Rev. A 50 (1994) 2960.

[57] P. G. Harris et al., Phys. Rev. Lett. 82 (1999) 904. 


\begin{tabular}{|c|c|c|c|c|}
\hline \multicolumn{5}{|c|}{ Luminosity table for $\mathrm{e}^{+} \mathrm{e}^{-} \rightarrow \mathcal{H Z}$ production } \\
\hline $\begin{array}{l}\text { Channel Name } \\
\mathcal{H Z} \rightarrow\end{array}$ & $\begin{array}{c}\text { Energies } \\
(\mathrm{GeV})\end{array}$ & $\begin{array}{c}\text { Luminosity } \\
\left(\mathrm{pb}^{-1}\right)\end{array}$ & $\begin{array}{l}\text { Mass range } \\
(\mathrm{GeV})\end{array}$ & $\begin{array}{l}\text { Described } \\
\text { in }\end{array}$ \\
\hline \multicolumn{5}{|c|}{ LEP 1 Channels } \\
\hline$\overline{\mathrm{qqq} \tau \tau / \tau \tau \mathrm{qq}}$ & 91.2 & 46.3 & $\bar{c}_{m_{\mathcal{H}}}=0-70$ & 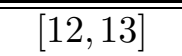 \\
\hline$\left(\mathcal{H}_{1} \mathcal{H}_{1} \rightarrow q \bar{q} q \bar{q}\right) \nu \nu$ & 91.2 & 46.3 & $m_{\mathcal{H}_{2}}=10-75, m_{\mathcal{H}_{1}}=0-35$ & 12,13 \\
\hline $\mathrm{qq} \nu \nu$ & 91.2 & 46.3 & $m_{\mathcal{H}}=0-70$ & 12,13 \\
\hline qqll & 91.2 & 46.3 & $m_{\mathcal{H}}=20-70$ & 12,13 \\
\hline \multicolumn{5}{|c|}{ LEP 2 Channels } \\
\hline bbqq & $10161-172$ & 20.4 & 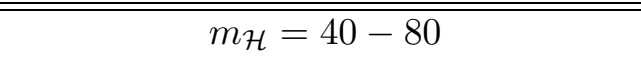 & 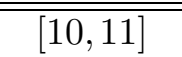 \\
\hline $\mathrm{bb} \nu \nu$ & $161-172$ & 20.4 & $m_{\mathcal{H}}=50-70$ & [10, 11 \\
\hline$\tau \tau \mathrm{qq}, \mathrm{bb} \tau \tau$ & $161-172$ & 20.4 & $m_{\mathcal{H}}=30-95$ & [10, 11 \\
\hline Xee & $161-172$ & 20.4 & $m_{\mathcal{H}}=35-80$ & [10, 11 \\
\hline $\mathrm{X} \mu \mu$ & $161-172$ & 20.4 & $m_{\mathcal{H}}=35-80$ & 10, 11 \\
\hline bbqq & 183 & 54.1 & $m_{\mathcal{H}}=40-95$ & 9 \\
\hline$\left(\mathcal{H}_{1} \mathcal{H}_{1} \rightarrow 4 \mathrm{~b}\right) \mathrm{qq}$ & 183 & 54.1 & $m_{\mathcal{H}_{2}}=40-80, m_{\mathcal{H}_{1}}=10.5-38$ & 9] \\
\hline $\mathrm{bb} \nu \nu /\left(\mathcal{H}_{1} \mathcal{H}_{1} \rightarrow 4 \mathrm{~b}\right) \nu \nu$ & 183 & 53.9 & $m_{\mathcal{H}}=50-95$ & 9] \\
\hline $\begin{array}{l}\mathrm{bb} \tau \tau / \\
\quad \tau \tau \mathrm{qq} /\left(\mathcal{H}_{1} \mathcal{H}_{1} \rightarrow 4 \mathrm{~b}\right) \tau \tau\end{array}$ & 183 & 53.7 & $m_{\mathcal{H}}=30-100$ & 9 \\
\hline bbee, bb $\mu \mu$ & 183 & 55.9 & $m_{\mathcal{H}}=60-100$ & 9 \\
\hline bbqq & 189 & 172.1 & $m_{\mathcal{H}}=40-100$ & 8 \\
\hline$\left(\mathcal{H}_{1} \mathcal{H}_{1} \rightarrow 4 \mathrm{~b}\right) \mathrm{qq}$ & 189 & 172.1 & $m_{\mathcal{H}_{2}}=40-100, m_{\mathcal{H}_{1}}=10.5-48$ & 8 \\
\hline $\mathrm{bb} \nu \nu /\left(\mathcal{H}_{1} \mathcal{H}_{1} \rightarrow 4 \mathrm{q}\right) \nu \nu$ & 189 & 171.4 & $m_{\mathcal{H}}=50-100$ & 8 \\
\hline $\begin{array}{l}\mathrm{bb} \tau \tau / \\
\quad \tau \tau \mathrm{qq} /\left(\mathcal{H}_{1} \mathcal{H}_{1} \rightarrow 4 \mathrm{~b}\right) \tau \tau\end{array}$ & 189 & 168.7 & $m_{\mathcal{H}}=30-100$ & 8 \\
\hline bbee, $\mathrm{bb} \mu \mu$ & 189 & 170.0 & $m_{\mathcal{H}}=70-100$ & 8 \\
\hline low $m_{\mathrm{A}}\left(\mathcal{H}_{1} \mathcal{H}_{1}\right)(\nu \bar{\nu}, \mathrm{ee}, \mu \mu)$ & 189-192 & 201.7 & $m_{\mathcal{H}_{2}}=45-90, m_{\mathcal{H}_{1}}=2-10.5$ & 29 \\
\hline bbqq & $192-209$ & 421.2 & $m_{\mathcal{H}}=80-120$ & 77 \\
\hline$\left(\mathcal{H}_{1} \mathcal{H}_{1} \rightarrow 4 \mathrm{~b}\right) \mathrm{qq}$ & $192-209$ & 421.2 & $m_{\mathcal{H}_{2}}=80-120, m_{\mathcal{H}_{1}}=12-m_{\mathcal{H}_{2}} / 2$ & 4.1 .1 \\
\hline $\mathrm{bb} \nu \nu$ & $192-209$ & 419.9 & $m_{\mathcal{H}}=30-120$ & 7 \\
\hline $\mathrm{bbbb} \nu \nu$ & 199-209 & 207.2 & $m_{\mathcal{H}_{2}}=100-110, m_{\mathcal{H}_{1}}=12-m_{\mathcal{H}_{2}} / 2$ & 4.1 .2 \\
\hline $\mathrm{bb} \tau \tau / \tau \tau \mathrm{qq}$ & $192-209$ & 417.4 & $m_{\mathcal{H}}=80-120$ & 7 \\
\hline bbee, $\mathrm{bb} \mu \mu$ & $192-209$ & 418.3 & $m_{\mathcal{H}}=40-120$ & 7 \\
\hline low $m_{\mathrm{A}}\left(\mathcal{H}_{1} \mathcal{H}_{1}\right)(\nu \bar{\nu}, \mathrm{ee}, \mu \mu)$ & $196-209$ & 396.9 & $m_{\mathcal{H}_{2}}=45-90, m_{\mathcal{H}_{1}}=2-10.5$ & 29 \\
\hline \multicolumn{5}{|c|}{ LEP 2 Flavour-Independent Channels } \\
\hline qqqq & 189 & 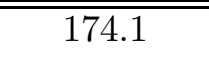 & 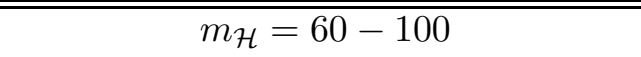 & [30] \\
\hline $\mathrm{qq} \nu \nu$ & 189 & 171.8 & $m_{\mathcal{H}}=30-100$ & 30 \\
\hline $\mathrm{qq} \tau \tau, \tau \tau \mathrm{qq}$ & 189 & 168.7 & $m_{\mathcal{H}}=30-100$ & 30 \\
\hline qqee, qq $\mu \mu$ & 189 & 170.0 & $m_{\mathcal{H}}=70-100$ & 30 \\
\hline qqqq & $192-209$ & 424.2 & $m_{\mathcal{H}}=60-120$ & 31 \\
\hline $\mathrm{qq} \nu \nu$ & $192-209$ & 414.5 & $m_{\mathcal{H}}=30-110$ & 31 \\
\hline $\mathrm{qq} \tau \tau, \tau \tau \mathrm{qq}$ & $192-209$ & 418.9 & $m_{\mathcal{H}}=60-115$ & 31 \\
\hline qqee, qq $\mu \mu$ & $192-209$ & 422.0 & $m_{\mathcal{H}}=60-120$ & 31 \\
\hline
\end{tabular}

Table 1: List of the searches for the Higgsstrahlung process. The last column gives the reference or section where the search is described. 


\begin{tabular}{|c|c|c|c|c|}
\hline \multicolumn{5}{|c|}{ Luminosity table for $\mathrm{e}^{+} \mathrm{e}^{-} \rightarrow \mathcal{H}_{1} \mathcal{H}_{2}$ production } \\
\hline $\begin{array}{l}\text { Channel Name } \\
\mathcal{H}_{1} \mathcal{H}_{2} \rightarrow\end{array}$ & $\begin{array}{c}\text { Energies } \\
(\mathrm{GeV})\end{array}$ & $\begin{array}{l}\text { Luminosity } \\
\left(\mathrm{pb}^{-1}\right)\end{array}$ & $\begin{array}{c}\text { Mass range } \\
(\mathrm{GeV})\end{array}$ & $\begin{array}{c}\text { Described } \\
\text { in }\end{array}$ \\
\hline \multicolumn{5}{|c|}{ LEP 1 Channels } \\
\hline $6 \mathrm{~b}$ & 91.2 & 27.6 & $\bar{m}_{m_{\mathcal{H}_{2}}}=40-70, m_{\mathcal{H}_{1}}=5-35$ & 12,13 \\
\hline $\mathrm{qq} \tau \tau, \tau \tau \mathrm{qq}$ & 91.2 & 46.3 & $m_{\mathcal{H}_{2}}=12-75, m_{\mathcal{H}_{1}}=10-78$ & 12,13 \\
\hline $6 \tau, 4 \tau 2 \mathrm{q}, 2 \tau 4 \mathrm{q}$ & 91.2 & 46.3 & $m_{\mathcal{H}_{2}}=30-75, m_{\mathcal{H}_{1}}=4-30$ & 12,13 \\
\hline \multicolumn{5}{|c|}{ LEEP 1.5 Channels } \\
\hline $4 \mathrm{bb}$ & $130-136$ & 5.2 & $\overline{\Sigma \Sigma}=80-130, \Delta=0-50$ & {$[11]$} \\
\hline $6 \mathrm{~b}$ & $130-136$ & 5.2 & $m_{\mathcal{H}_{2}}=55-65, m_{\mathcal{H}_{1}}>27.5$ & 11 \\
\hline \multicolumn{5}{|c|}{ LEP 2 Channels } \\
\hline 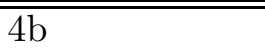 & 161 & 10.0 & $\overline{\Sigma \Sigma}=80-130, \Delta=0-60$ & 10,11 \\
\hline $6 \mathrm{~b}$ & 161 & 10.0 & $m_{\mathcal{H}_{2}}=55-65, m_{\mathcal{H}_{1}}>20.0$ & 10,11 \\
\hline $\mathrm{bb} \tau \tau, \tau \tau \mathrm{bb}$ & 161 & 10.0 & $m_{\mathcal{H}_{2}}=40-160, m_{\mathcal{H}_{1}}=52-160$ & 10,11 \\
\hline $4 \mathrm{~b}$ & 172 & 10.4 & $\Sigma=80-130, \Delta=0-60$ & 10,11 \\
\hline $6 \mathrm{~b}$ & 172 & 10.4 & $m_{\mathcal{H}_{2}}=55-65, m_{\mathcal{H}_{1}}=25-35$ & 10,11 \\
\hline $\mathrm{bb} \tau \tau, \tau \tau \mathrm{bb}$ & 172 & 10.4 & $m_{\mathcal{H}_{2}}=37-160, m_{\mathcal{H}_{1}}=28-160$ & 10, 11 \\
\hline $4 \mathrm{~b}$ & 183 & 54.1 & $\Sigma=80-150, \Delta=0-60$ & 9] \\
\hline $6 b$ & 183 & 54.1 & $m_{\mathcal{H}_{2}}=30-80, m_{\mathcal{H}_{1}}=12-40$ & 9] \\
\hline $\mathrm{bb} \tau \tau, \tau \tau \mathrm{bb}$ & 183 & 53.7 & $\Sigma=70-170, \Delta=0-70$ & 9 \\
\hline $4 \mathrm{~b}$ & 189 & 172.1 & $\Sigma=80-180, \Delta=0-70$ & 8 \\
\hline $6 \mathrm{~b}$ & 189 & 172.1 & $m_{\mathcal{H}_{2}}=24-80, m_{\mathcal{H}_{1}}=12-40$ & 8 \\
\hline $\mathrm{bb} \tau \tau, \tau \tau \mathrm{bb}$ & 189 & 168.7 & $\Sigma=70-190, \Delta=0-90$ & 8 \\
\hline $4 \mathrm{~b}$ & 192 & 28.9 & $\Sigma=83-183, \Delta=0-70$ & 4.2 .1 \\
\hline $4 \mathrm{~b}$ & 196 & 74.8 & $\Sigma=80-187, \Delta=0-70$ & 4.2 .1 \\
\hline $4 \mathrm{~b}$ & 200 & 77.2 & $\Sigma=80-191, \Delta=0-70$ & 4.2 .1 \\
\hline $4 \mathrm{~b}$ & 202 & 36.1 & $\Sigma=80-193, \Delta=0-70$ & 4.2 .1 \\
\hline high $m_{\mathcal{H}_{1}} 4 \mathrm{~b}$ & 199-209 & 207.3 & $\Sigma=120-190, \Delta=0-70$ & 4.2 .1 \\
\hline low $m_{\mathcal{H}_{1}} 4 \mathrm{~b}$ & 199-209 & 207.3 & $\Sigma=100-140, \Delta=60-100$ & 4.2 .2 \\
\hline & 199-209 & 207.3 & $\Sigma=90-200, \Delta=40-160$ & 4.2 .3 \\
\hline $\mathrm{bb} \tau \tau, \tau \tau \mathrm{bb}$ & 192 & 28.7 & $\Sigma=10-174, \Delta=0-182$ & 4.2 .4 \\
\hline $\mathrm{bb} \tau \tau, \tau \tau \mathrm{bb}$ & 196 & 74.7 & $\Sigma=10-182, \Delta=0-191$ & 4.2 .4 \\
\hline $\mathrm{bb} \tau \tau, \tau \tau \mathrm{bb}$ & 200 & 74.8 & $\Sigma=10-182, \Delta=0-191$ & 4.2 .4 \\
\hline $\mathrm{bb} \tau \tau, \tau \tau \mathrm{bb}$ & 202 & 35.4 & $\Sigma=10-174, \Delta=0-182$ & 4.2 .4 \\
\hline $\mathrm{bb} \tau \tau, \tau \tau \mathrm{bb}$ & 199-209 & 203.6 & $\Sigma=70-190, \Delta=0-90$ & 4.2 .4 \\
\hline
\end{tabular}

Table 2: List of the searches for pair production. The last column gives the reference or section where the search is described. The symbols $\Sigma=m_{\mathcal{H}_{1}}+m_{\mathcal{H}_{2}}$ and $\Delta=m_{\mathcal{H}_{2}}-m_{\mathcal{H}_{1}}$ denote the Higgs mass sum and difference. 


\begin{tabular}{|c|c|c|c|c|c|c|}
\hline \multirow{2}{*}{$\begin{array}{c}m_{\mathcal{H}_{2}} \\
(\mathrm{GeV})\end{array}$} & \multirow{2}{*}{$\begin{array}{c}m_{\mathcal{H}_{1}} \\
(\mathrm{GeV})\end{array}$} & \multicolumn{5}{|c|}{ Efficiency for the process $\mathcal{H}_{2} \mathrm{Z} \rightarrow \mathrm{bbbbq} \overline{\mathrm{q}}$ at $\sqrt{s}$} \\
\hline & & $192 \mathrm{GeV}$ & $196 \mathrm{GeV}$ & $200 \mathrm{GeV}$ & $202 \mathrm{GeV}$ & $206 \mathrm{GeV}$ \\
\hline 100. & 12. & 0.689 & 0.684 & 0.717 & 0.733 & 0.693 \\
\hline 100. & 20. & 0.651 & 0.639 & 0.653 & 0.659 & 0.586 \\
\hline 100. & 30 . & 0.460 & 0.461 & 0.461 & 0.470 & 0.480 \\
\hline 100. & 40. & 0.270 & 0.260 & 0.283 & 0.315 & 0.323 \\
\hline 100. & 48. & 0.328 & 0.325 & 0.361 & 0.392 & 0.400 \\
\hline 105. & 12. & 0.538 & 0.658 & 0.702 & 0.709 & 0.701 \\
\hline 105. & 20. & 0.562 & 0.618 & 0.697 & 0.658 & 0.681 \\
\hline 105. & 30 . & 0.490 & 0.525 & 0.509 & 0.536 & 0.497 \\
\hline 105. & 40. & 0.407 & 0.306 & 0.309 & 0.316 & 0.319 \\
\hline 105. & 50. & 0.433 & 0.368 & 0.355 & 0.359 & 0.370 \\
\hline 110. & 12. & & & 0.637 & 0.682 & 0.720 \\
\hline 110. & 20. & & & 0.625 & 0.646 & 0.532 \\
\hline 110. & 30. & & & 0.556 & 0.549 & 0.565 \\
\hline 110. & 40. & & & 0.380 & 0.328 & 0.343 \\
\hline 110. & 53. & & & 0.395 & 0.341 & 0.358 \\
\hline
\end{tabular}

Table 3: Efficiencies of the standard $\mathrm{e}^{+} \mathrm{e}^{-} \rightarrow \mathrm{ZH} \rightarrow \mathrm{q} \overline{\mathrm{q}} \mathrm{b} \overline{\mathrm{b}}$ analysis [7] for the $\mathrm{e}^{+} \mathrm{e}^{-} \rightarrow \mathrm{ZH}_{2} \rightarrow \mathrm{ZH}_{1} \mathcal{H}_{1} \rightarrow \mathrm{q} \overline{\mathrm{q}} \mathrm{b} \overline{\mathrm{b}} \mathrm{b} \overline{\mathrm{b}}$ final state (see Section 4.1.1). The uncertainties from Monte Carlo statistics are of the order of \pm 0.015 .

\begin{tabular}{|c|c|c|c|c|c|c|c|}
\hline cut & data & tot. bkg. & $\mathrm{qq}(\gamma)$ & tot $4-f$ & $\begin{array}{c}\text { Eff. } \\
\mathcal{H}_{2} \rightarrow \mathcal{H}_{1} \mathcal{H}_{1} \\
m_{\mathcal{H}_{2}}=105 \\
m_{\mathcal{H}_{1}}=20\end{array}$ & $\begin{array}{c}\text { Eff. } \\
\mathcal{H}_{2} \rightarrow \mathcal{H}_{1} \mathcal{H}_{1} \\
m_{\mathcal{H}_{2}}=105 \\
m_{\mathcal{H}_{1}}=40\end{array}$ & $\begin{array}{c}\text { Eff. } \\
\mathcal{H}_{2} \rightarrow \mathrm{b} \bar{b} \\
m_{\mathcal{H}_{2}}=105\end{array}$ \\
\hline$(1)-(5)$ & 503 & 424.77 & 123.94 & 297.99 & 0.75 & 0.74 & 0.59 \\
\hline \multicolumn{8}{|c|}{ 2-jet topology subsample A } \\
\hline$(6)$ & 371 & 308.56 & 108.66 & 197.17 & 0.75 & 0.16 & 0.53 \\
\hline (7) & 213 & 201.77 & 24.70 & 177.07 & 0.70 & 0.15 & 0.50 \\
\hline (8) & 135 & 126.29 & 22.85 & 103.43 & 0.68 & 0.14 & 0.49 \\
\hline $\mathrm{ANN}_{\mathrm{A}}$ & 11 & 10.0 & 2.63 & 7.39 & 0.59 & 0.11 & 0.40 \\
\hline \multicolumn{8}{|c|}{ 4-jet topology subsample B } \\
\hline$(6)$ & 118 & 112.32 & 14.42 & 97.80 & 0.008 & 0.57 & 0.06 \\
\hline $\mathrm{ANN}_{\mathrm{B}}$ & 8 & 7.20 & 2.83 & 4.37 & 0.003 & 0.55 & 0.05 \\
\hline \multicolumn{8}{|c|}{ Total $(2+4$ jets $)$} \\
\hline Sum & 19 & $17.2 \pm 0.6$ & 5.46 & 11.8 & 0.59 & 0.66 & 0.45 \\
\hline
\end{tabular}

Table 4: Cut flow in the missing energy analysis for $\mathcal{H}_{2} \nu \bar{\nu} \rightarrow \mathrm{b} \overline{\mathrm{b}} \nu \bar{\nu}$ and $\mathcal{H}_{2} \nu \bar{\nu} \rightarrow \mathcal{H}_{1} \mathcal{H}_{1} \nu \bar{\nu} \rightarrow \mathrm{b} \bar{b} b \bar{b} \nu \bar{\nu}$ for $100 \leq m_{\mathcal{H}_{2}} \leq 110 \mathrm{GeV}$ for data taken at $\sqrt{s}=199$ to $209 \mathrm{GeV}$ (see Section 4.1.2). The uncertainty quoted for the total background is from Monte Carlo statistics only. 


\begin{tabular}{|c|c|c|c|}
\hline \multicolumn{4}{|c|}{ Efficiency $\mathrm{A}$ and $\mathrm{B}$ for $\mathcal{H}_{2} \nu \bar{\nu} \rightarrow \mathrm{bb} \nu \bar{\nu}$ decays } \\
\hline \multicolumn{2}{|c|}{$m_{\mathcal{H}_{2}}(\mathrm{GeV})$} & Efficiency of selection A & Efficiency of selection B \\
\hline \multicolumn{2}{|c|}{100} & 0.382 & 0.044 \\
\hline \multicolumn{2}{|c|}{105.} & 0.396 & 0.050 \\
\hline \multicolumn{2}{|c|}{110.} & 0.379 & 0.050 \\
\hline \multicolumn{4}{|c|}{ Efficiency $\mathrm{A}$ and $\mathrm{B}$ for $\mathcal{H}_{2} \nu \bar{\nu} \rightarrow \mathcal{H}_{1} \mathcal{H}_{1} \nu \bar{\nu} \rightarrow \mathrm{bbbb} \nu \bar{\nu}$ decays } \\
\hline$m_{\mathcal{H}_{2}}(\mathrm{GeV})$ & $m_{\mathcal{H}_{1}}(\mathrm{GeV})$ & Efficiency of selection A & Efficiency of selection B \\
\hline 100 & 12. & 0.686 & 0.0 \\
\hline 100. & 20. & 0.561 & 0.001 \\
\hline 100. & 30 . & 0.280 & 0.301 \\
\hline 100. & 40. & 0.090 & 0.522 \\
\hline 100. & 48. & 0.195 & 0.436 \\
\hline 105. & 12. & 0.707 & 0.0 \\
\hline 105. & 20. & 0.587 & 0.0 \\
\hline 105. & 30 . & 0.349 & 0.254 \\
\hline 105. & 40. & 0.113 & 0.550 \\
\hline 105. & 50. & 0.179 & 0.487 \\
\hline 110. & 12. & 0.677 & 0.0 \\
\hline 110 . & 20. & 0.585 & 0.001 \\
\hline 110. & 30 . & 0.402 & 0.189 \\
\hline 110. & 40. & 0.109 & 0.555 \\
\hline 110. & 50. & 0.131 & 0.537 \\
\hline 110. & 53. & 0.186 & 0.495 \\
\hline
\end{tabular}

Table 5: Efficiencies of the selections $A$ and $B$ of the missing energy analysis for $\mathcal{H}_{2} \rightarrow \mathrm{b} \bar{b} \nu \bar{\nu}$ and $\mathcal{H}_{2} \rightarrow \mathcal{H}_{1} \mathcal{H}_{1} \rightarrow \mathrm{b} \bar{b} b \bar{b} \nu \bar{\nu}$ for $100 \leq m_{\mathcal{H}_{2}} \leq 110 \mathrm{GeV}$ for data taken at $\sqrt{s}=199$ to $209 \mathrm{GeV}$ is used (see Section 4.1.2). The uncertainty from Monte Carlo statistics is of the order of \pm 0.010 . 


\begin{tabular}{|c|c|c|c|c|c|}
\hline Cut & Data & Total bkg. & $\overline{q \bar{q}}(\gamma)$ & 4 4-fermi. & $\begin{array}{c}\text { Efficiency (\%) } \\
m_{\mathcal{H}_{1}}=m_{\mathcal{H}_{2}}=90 \mathrm{GeV} \\
\end{array}$ \\
\hline \multicolumn{6}{|c|}{$\mathcal{H}_{2} \mathcal{H}_{1} \rightarrow 4$ b Channel } \\
\hline (1) & 39367 & 39375.6 & 30958.7 & 8325.6 & 99.8 \\
\hline (2) & 13792 & 13895.2 & 8914.4 & 4976.0 & 98.4 \\
\hline (3) & 4682 & 4509.6 & 1110.5 & 3397.9 & 88.3 \\
\hline (4) & 3997 & 3994.5 & 707.7 & 3285.7 & 86.4 \\
\hline (5) & 3474 & 3431.0 & 566.3 & 2863.6 & 85.6 \\
\hline (6) & 3331 & 3271.5 & 520.4 & 2749.9 & 83.7 \\
\hline $\mathcal{L}^{\mathcal{H}_{1} \mathcal{H}_{2}}>0.95$ & 22 & $19.9 \pm 0.3$ & 6.5 & 13.4 & 49.4 \\
\hline \multicolumn{6}{|c|}{$\overline{\overline{\mathcal{H}_{2}} \mathcal{H}_{1} \rightarrow \mathrm{bb} \tau^{+} \tau^{-} \text {Channel }}$} \\
\hline Pre-sel & 336.0 & 354.6 & 96.0 & 258.5 & 53.8 \\
\hline $\mathcal{L}^{\mathcal{H}_{2} \mathcal{H}_{1}}>0.64$ & $\overline{13}$ & $13.2 \pm 0.4$ & 0.7 & 12.4 & 42.5 \\
\hline
\end{tabular}

Table 6: Cut flow in the $\mathcal{H}_{1} \mathcal{H}_{2}$ channels for high $m_{\mathcal{H}_{1}}$ (see Section 4.2) and for all data taken at $\sqrt{s}=192$ to $209 \mathrm{GeV}$ : effect of the cuts on the data and the background, normalised to the integrated luminosity of the data. The two-photon background, not shown separately, is included in the total background. The signal efficiencies are given in the last column for $m_{\mathcal{H}_{1}}=m_{\mathcal{H}_{2}}=90 \mathrm{GeV}$.

\begin{tabular}{|c||c|c|c|c|c|c|c|c|}
\hline \multicolumn{1}{|c||}{} & \multicolumn{7}{c|}{ Efficiency for the process $\mathcal{H}_{2} \mathcal{H}_{1} \rightarrow$ bbbb (high $m_{\mathcal{H}_{1}}$ ) at } \\
\hline \multicolumn{1}{|c||}{} & \multicolumn{7}{c|}{$=206 \mathrm{GeV}$} \\
\hline \hline$m_{\mathcal{H}_{1}}(\mathrm{GeV})$ & 30.0 & 40.0 & 50.0 & 60.0 & 70.0 & 80.0 & 90.0 \\
\hline \hline$m_{\mathcal{H}_{2}}(\mathrm{GeV})$ & \multicolumn{7}{|c|}{} \\
\hline 30.0 & 0.001 & & & & & & \\
40.0 & 0.0008 & 0.004 & & & & & \\
50.0 & & 0.110 & 0.215 & & & & \\
60.0 & 0.103 & & 0.274 & 0.364 & & & \\
70.0 & & 0.254 & & 0.381 & 0.388 & & \\
80.0 & 0.200 & & 0.384 & & 0.425 & 0.470 & \\
90.0 & & 0.319 & & 0.388 & & 0.472 & 0.479 \\
100.0 & & & 0.374 & & 0.432 & & 0.435 \\
110.0 & & 0.341 & & 0.371 & & & \\
120.0 & 0.261 & & 0.349 & & 0.399 & & \\
130.0 & & 0.253 & & 0.368 & & & \\
140.0 & 0.231 & & 0.290 & & & & \\
150.0 & & 0.177 & & & & & \\
160.0 & 0.116 & & & & &
\end{tabular}

Table 7: Efficiencies of the $\mathcal{H}_{1} \mathcal{H}_{2} \rightarrow \mathrm{b} \bar{b} b \bar{b}$ analysis for high $m_{\mathcal{H}_{1}}$ (see Section 4.2.1). The uncertainty from Monte Carlo statistics is typically of the order of \pm 0.015 . The table is showing the Monte Carlo points produced. 


\begin{tabular}{|l||c|c|c|c||c|c|}
\hline \multicolumn{7}{|c|}{ Systematic uncertainties at $\sqrt{s}=206 \mathrm{GeV}$} \\
\hline \multicolumn{1}{|c||}{} & $\mathcal{H}_{2} \mathcal{H}_{1} \rightarrow$ bbbb (high $m_{\mathcal{H}_{1}}$ ) & \multicolumn{1}{|c|}{$\mathcal{H}_{2} \mathcal{H}_{1} \rightarrow$ bbbb (low $\left.m_{\mathcal{H}_{1}}\right)$} & \multicolumn{1}{|c|}{$\mathcal{H}_{2} \mathcal{H}_{1} \rightarrow \mathrm{bb} \tau^{+} \tau^{-}, \tau^{+} \tau^{-}$bb } \\
\hline Source & Signal eff. & Background & Signal eff. & Background & Signal eff. & Background \\
\hline \hline Detector modelling & $0.9 \%$ & $8.0 \%$ & $1.1 \%$ & $8.6 \%$ & $1.0 \%$ & $1.0 \%$ \\
B-had. Decay Mult. & $0.9 \%$ & $1.2 \%$ & $2.1 \%$ & $1.9 \%$ & $1.0 \%$ & $3.0 \%$ \\
B-had. Fragment. & $1.8 \%$ & $1.5 \%$ & $2.7 \%$ & $1.5 \%$ & $1.8 \%$ & $1.5 \%$ \\
C-had. Fragment. & $0.0 \%$ & $0.5 \%$ & $0.0 \%$ & $0.0 \%$ & $0.0 \%$ & $0.0 \%$ \\
4f-cross-section & $0.0 \%$ & $1.6 \%$ & $0.0 \%$ & $1.0 \%$ & $0.0 \%$ & $1.9 \%$ \\
MC-Generators & $0.0 \%$ & $2.6 \%$ & $0.0 \%$ & $2.6 \%$ & $0.0 \%$ & $0.0 \%$ \\
$\tau$ identification & $0.0 \%$ & $0.0 \%$ & $0.0 \%$ & $0.0 \%$ & $0.9 \%$ & $15.0 \%$ \\
LH modelling & $0.9 \%$ & $2.3 \%$ & $1.0 \%$ & $2.1 \%$ & $0.0 \%$ & $0.0 \%$ \\
MC statistics & $2.0 \%$ & $5.0 \%$ & $3.0 \%$ & $5.2 \%$ & $1.0 \%$ & $3.0 \%$ \\
\hline Combined & $3.1 \%$ & $10.3 \%$ & $4.7 \%$ & $10.9 \%$ & $2.6 \%$ & $15.8 \%$ \\
\hline
\end{tabular}

Table 8: Systematic uncertainties on the signal efficiency and background at $\sqrt{s}=206 \mathrm{GeV}$ for the processes $\mathcal{H}_{1} \mathcal{H}_{2} \rightarrow \mathrm{b} \overline{\mathrm{b}} \overline{\mathrm{b}}$ with high $m_{\mathcal{H}_{1}}$, low $m_{\mathcal{H}_{1}}$ and for $\mathcal{H}_{2} \mathcal{H}_{1} \rightarrow \mathrm{b} \overline{\mathrm{b}} \tau^{+} \tau^{-}, \tau^{+} \tau^{-} \mathrm{b} \overline{\mathrm{b}}$ (see Sections 4.2.1, 4.2.2 and 4.2.4).

\begin{tabular}{|c||r||r||r|r||c|}
\hline Cut & Data & Total bkg. & $\mathrm{q} \overline{\mathrm{q}}(\gamma)$ & 4-fermi. & $\begin{array}{c}\text { Efficiency }(\%) \\
m_{\mathcal{H}_{1}}=30, m_{\mathcal{H}_{2}}=100 \mathrm{GeV}\end{array}$ \\
\hline \hline \multicolumn{5}{|c||}{} & \multicolumn{1}{|c|}{$\mathcal{H}_{1} \mathcal{H}_{2} \rightarrow 4$ b Channel for low $m_{\mathcal{H}_{1}}$} \\
\hline$(1)$ & 18519 & 17802.0 & 13705.2 & 4096.9 & 99.6 \\
$(2)$ & 6538 & 6427.8 & 3971.6 & 2456.3 & 96.2 \\
$(3)$ & 4215 & 4048.0 & 2082.4 & 1965.6 & 94.2 \\
$(4)$ & 3618 & 3497.5 & 1546.8 & 1950.6 & 93.6 \\
$(5)$ & 2712 & 2625.9 & 1188.5 & 1437.4 & 90.5 \\
$(6)$ & 2477 & 2389.4 & 1060.0 & 1329.5 & 83.5 \\
\hline $\mathcal{L}^{\mathcal{H}_{1} \mathcal{H}_{2}}>0.98$ & 8 & $10.4 \pm 0.1$ & 6.1 & 4.3 & 36.9 \\
\hline
\end{tabular}

Table 9: Cut flow in the $\mathcal{H}_{1} \mathcal{H}_{2}$ channel for low $m_{\mathcal{H}_{1}}$ (see Section 4.2.2) and for all data taken at $\sqrt{s}=199$ to $209 \mathrm{GeV}$ : effect of the cuts on the data and the background, normalized to the integrated luminosity of the data. The two-photon background, not shown separately, is included in the total background. The signal efficiencies are given in the last column for $m_{\mathcal{H}_{1}}=30 \mathrm{GeV}$ and $m_{\mathcal{H}_{2}}=100 \mathrm{GeV}$ in the $\mathcal{H}_{1} \mathcal{H}_{2} \rightarrow \mathrm{bb} \bar{b}$ channel.

\begin{tabular}{|c|c|c|c|}
\hline \multicolumn{4}{|c|}{$\begin{array}{l}\text { Efficiency for the process } \mathcal{H}_{2} \mathcal{H}_{1} \rightarrow \text { bbbb (low } m_{\mathcal{H}_{1}} \text { ) a } \\
\qquad \sqrt{s}=206 \mathrm{GeV}\end{array}$} \\
\hline $\bar{m}_{\mathcal{H}_{1}}(\mathrm{GeV})$ & 12.0 & 20.0 & 30.0 \\
\hline \multicolumn{4}{|l|}{$m_{\mathcal{H}_{2}}(\mathrm{GeV})$} \\
\hline 90. & 0.269 & 0.330 & 0.370 \\
\hline 95. & 0.286 & 0.341 & 0.384 \\
\hline 100. & 0.305 & 0.366 & 0.369 \\
\hline 105. & 0.310 & 0.358 & 0.369 \\
\hline 110. & 0.298 & 0.351 & 0.366 \\
\hline
\end{tabular}

Table 10: Efficiencies of the $\mathcal{H}_{1} \mathcal{H}_{2} \rightarrow \mathrm{b} \bar{b} b \bar{b}$ analysis for low $m_{\mathcal{H}_{1}}$ (see Section 4.2.2). The uncertainty from Monte Carlo statistics is of the order of \pm 0.011 . 


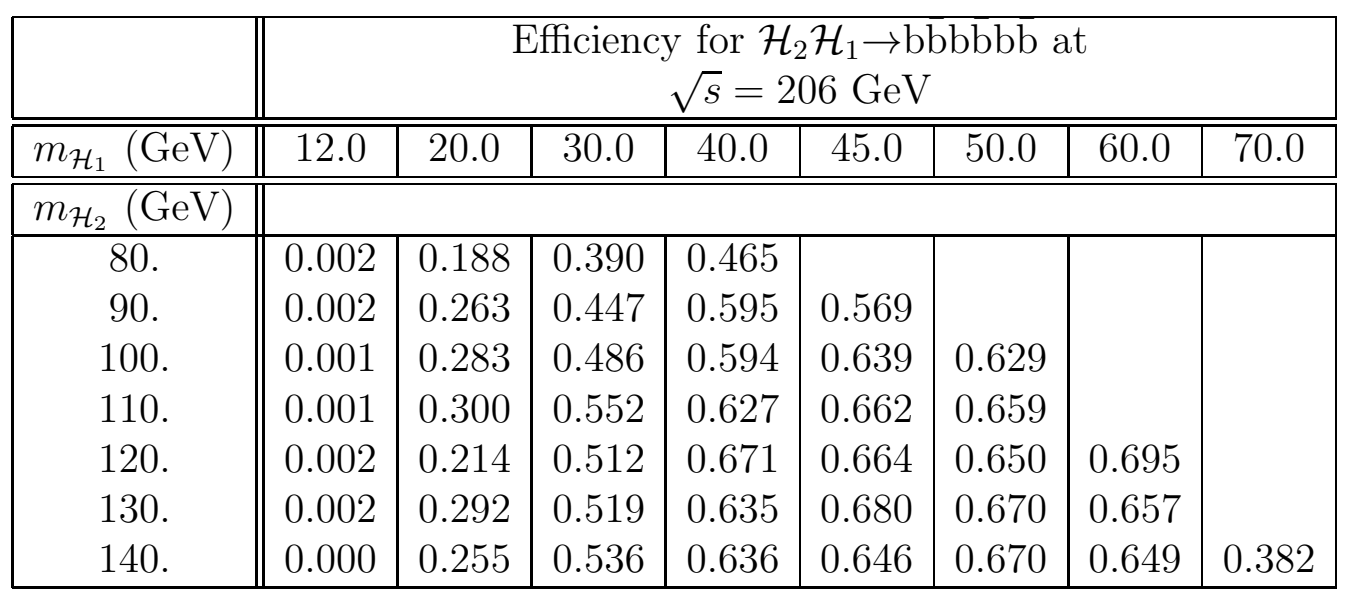

Table 11: Efficiencies of the $\mathcal{H}_{1} \mathcal{H}_{2} \rightarrow \mathcal{H}_{1} \mathcal{H}_{1} \mathcal{H}_{1} \rightarrow \mathrm{b} \bar{b} b \bar{b} b \bar{b}$ analysis (see Section 4.2.3). The uncertainty from Monte Carlo statistics is \pm 0.010 .

\begin{tabular}{|c|c|c|c|c|c|c|c|}
\hline & \multicolumn{7}{|c|}{$\begin{array}{c}\text { Efficiency for } \mathcal{H}_{2} \mathcal{H}_{1} \rightarrow \mathrm{bb} \tau^{+} \tau^{-} \text {at } \\
\sqrt{s}=206 \mathrm{GeV}\end{array}$} \\
\hline$\overline{m_{\mathcal{H}_{1}}(\mathrm{GeV})}$ & 30. & 40. & 50. & 60. & 70. & 80. & 90. \\
\hline \multicolumn{8}{|l|}{$m_{\mathcal{H}_{2}}(\mathrm{GeV})$} \\
\hline \multirow{15}{*}{$\begin{array}{c}30 . \\
40 . \\
50 . \\
60 . \\
70 . \\
80 . \\
90 \\
100 \\
110 . \\
120 . \\
130 \\
140 . \\
150 \\
160 \\
170\end{array}$} & 0.0818 & \multirow{3}{*}{$\begin{array}{l}0.2024 \\
0.3296\end{array}$} & \multirow[b]{3}{*}{0.3690} & \multirow[b]{4}{*}{0.3668} & \multirow[b]{5}{*}{0.4631} & \multirow{6}{*}{0.4247} & \multirow{15}{*}{0.4305} \\
\hline & 0.1016 & & & & & & \\
\hline & & & & & & & \\
\hline & 0.3152 & & 0.4086 & & & & \\
\hline & & 0.3802 & & 0.3936 & & & \\
\hline & 0.4265 & \multirow[b]{2}{*}{0.3599} & 0.3856 & \multirow[b]{2}{*}{0.4285} & 0.4343 & & \\
\hline & \multirow{3}{*}{0.3854} & & & & \multirow{3}{*}{0.3951} & 0.3925 & \\
\hline & & & 0.4367 & & & 0.4298 & \\
\hline & & \multirow[t]{2}{*}{0.3628} & & 0.4227 & & & \\
\hline & \multirow[t]{2}{*}{0.3631} & & 0.3609 & & \multirow[t]{2}{*}{0.4020} & & \\
\hline & & \multirow[t]{2}{*}{0.2996} & & 0.3180 & & & \\
\hline & & & 0.3049 & & & & \\
\hline & & \multirow[t]{2}{*}{0.2278} & & & & & \\
\hline & & & & & & & \\
\hline & & & & & & & \\
\hline
\end{tabular}

Table 12: Efficiencies of the $\mathcal{H}_{1} \mathcal{H}_{2} \rightarrow \mathrm{b} \overline{\mathrm{b}} \tau^{+} \tau^{-}$analysis (see Section 4.2.4). The uncertainty from Monte Carlo statistics is of the order of \pm 0.02 .

\begin{tabular}{|l||c|c|c|}
\hline Channel & $\begin{array}{c}\text { Total } \\
\text { Background }\end{array}$ & Data & Section \\
\hline \hline $\mathcal{H}_{2} \mathrm{Z} \rightarrow \mathrm{bb}(\mathrm{bb}) \mathrm{q} \overline{\mathrm{q}}$ & 135.4 & 140 & 4.1 .1 \\
$\mathcal{H}_{2} \nu \bar{\nu} \rightarrow \mathrm{b} \overline{\mathrm{b}}(\mathrm{b} \overline{\mathrm{b}}) \nu \bar{\nu}$ & 39.5 & 36 & 4.1 .2 \\
$\mathcal{H}_{2} \mathcal{H}_{1} \rightarrow \mathrm{b} \bar{b} b \bar{b}(\mathrm{~b} \overline{\mathrm{b}})$ & 19.9 & 22 & $4.2 .1,4.2 .2,4.2 .3$ \\
$\mathcal{H}_{2} \mathcal{H}_{1} \rightarrow \mathrm{b} \overline{\mathrm{b}} \tau^{+} \tau^{-}$ & 13.2 & 13 & 4.2 .4 \\
\hline
\end{tabular}

Table 13: Typical numbers of background and data for the searches described in this publication. 


\begin{tabular}{|c|c|c|c|c|c|c|}
\hline Parameter & $\begin{array}{c}\text { no mixing } \\
/(\text { no-mixing }(2 \mathrm{TeV}))\end{array}$ & $\begin{array}{c}m_{\mathrm{h}}-\max \\
/\left(m_{\mathrm{h}}-\max ^{+}\right) \\
/\left(\mathrm{C} \mathrm{m}_{\mathrm{h}}-\max \right)\end{array}$ & large- $\mu$ & gluophobic & small $\alpha_{\text {eff }}$ & $\mathrm{CPX}$ \\
\hline \multicolumn{7}{|c|}{ Parameters varied in the scan } \\
\hline $\tan \beta$ & $\begin{array}{c}0.4-40 \\
/(0.7-40)\end{array}$ & $0.4-40$ & $0.7-40$ & $0.4-40$ & $0.4-40$ & $0.6-40$ \\
\hline$m_{\mathrm{A}}(\mathrm{GeV})$ & $0-1000$ & $0-1000$ & $0-400$ & $0-1000$ & $0-1000$ & - \\
\hline$m_{\mathrm{H}^{ \pm}}(\mathrm{GeV})$ & - & - & - & - & - & $4-1000$ \\
\hline \multicolumn{7}{|c|}{ Fixed parameters } \\
\hline$m_{\mathrm{t}}(\mathrm{GeV})$ & 174.3 & 174.3 & 174.3 & 174.3 & 174.3 & 174.3 \\
\hline$m_{\mathrm{SUSY}}(\mathrm{GeV})$ & $\begin{array}{l}1000 \\
/(2000)\end{array}$ & 1000 & 400 & 350 & 800 & 500 \\
\hline$M_{2}(\mathrm{GeV})$ & 200 & 200 & 400 & 300 & 500 & 200 \\
\hline$\mu(\mathrm{GeV})$ & $\begin{array}{l}-200 \\
/(200)\end{array}$ & $\begin{array}{l}-200 \\
/(200) \\
/(200)\end{array}$ & 1000 & 300 & 2000 & 2000 \\
\hline$m_{\tilde{\mathrm{g}}}(\mathrm{GeV})$ & 800 & 800 & 200 & 500 & 500 & 1000 \\
\hline$X_{\mathrm{t}}(\mathrm{GeV})$ & 0 & $\begin{array}{c}\sqrt{6} m_{\mathrm{SUSY}} \\
/\left(\sqrt{6} m_{\mathrm{SUSY}}\right) \\
/\left(-\sqrt{6} m_{\mathrm{SUSY}}\right)\end{array}$ & -300 & -750 & -1100 & $A_{\mathrm{t}}-\mu \cot \beta$ \\
\hline$A_{\mathrm{t}, \mathrm{b}}(\mathrm{GeV})$ & $X_{\mathrm{t}}+\mu \cot \beta$ & $X_{\mathrm{t}}+\mu \cot \beta$ & $X_{\mathrm{t}}+\mu \cot \beta$ & $X_{\mathrm{t}}+\mu \cot \beta$ & $X_{\mathrm{t}}+\mu \cot \beta$ & 1000 \\
\hline $\arg \left(A_{\mathrm{t}, \mathrm{b}}\right)$ & 0 & 0 & 0 & 0 & 0 & $90^{\circ}$ \\
\hline $\arg \left(m_{\tilde{\mathrm{g}}}\right)$ & 0 & 0 & 0 & 0 & 0 & $90^{\circ}$ \\
\hline
\end{tabular}

Table 14: Parameters of benchmark scenarios considered. Note that the values for $X_{\mathrm{t}}$ and $A_{\mathrm{t}, \mathrm{b}}$ are given for the $\overline{\mathrm{MS}}$-renormalization scheme. For a description of the choice of parameters see Section 7 Columns 2 to 6 refer to the CPC benchmark sets and the last column refers to the basic CPV benchmark set CPX.

\begin{tabular}{|l|c|c|c|}
\hline \multicolumn{4}{|c|}{ Limits on the MSSM scenarios } \\
\hline Benchmark set & Lower limit on $m_{\mathrm{h}}(\mathrm{GeV})$ & Lower limit on $m_{\mathrm{A}}(\mathrm{GeV})$ & Excluded $\tan \beta$ \\
\hline no mixing & $64.0(60.0)$ & - & $0.8<\tan \beta<6.2(0.9<\tan \beta<7.2)$ \\
no mixing $(2 \mathrm{TeV})$ & $83.3(88.0)$ & $84.3(88.8)$ & $0.9<\tan \beta<4.2(0.9<\tan \beta<4.3)$ \\
$m_{\mathrm{h}}-$ max & $84.5(88.5)$ & $85.0(89.0)$ & $0.7<\tan \beta<1.9(0.7<\tan \beta<1.9)$ \\
$m_{\mathrm{h}}-$ max $^{+}$ & $84.5(88.0)$ & $84.0(89.5)$ & $0.7<\tan \beta<1.9(0.7<\tan \beta<1.9)$ \\
constr. $m_{\mathrm{h}}-\max$ & $84.0(88.0)$ & $85.0(89.0)$ & $0.6<\tan \beta<2.2(0.6<\tan \beta<2.2)$ \\
gluophobic & $82.0(87.0)$ & $87.5(90.5)$ & $\tan \beta<6.0(\tan \beta<8.0)$ \\
small $\alpha_{\text {eff }}$ & $79.0(83.0)$ & $90.0(95.0)$ & $0.4<\tan \beta<3.6(0.4<\tan \beta<3.6)$ \\
CPX & - & - & $\tan \beta<2.8(\tan \beta<2.8)$ \\
\hline \hline \multicolumn{3}{|c|}{ Allowed regions in the "large $\mu$ " scenario } & $\tan \beta>6$ \\
\hline large $\mu$ & $80.0<m_{\mathrm{h}}<107.0$ & $87.0<m_{\mathrm{A}}$ & $(\tan \beta>12)$ \\
\hline
\end{tabular}

Table 15: Limits on $m_{\mathrm{h}} m_{\mathrm{A}}$ and $\tan \beta$ for the various benchmark sets. The median expected limits in an ensemble of SM background-only experiments are listed in parentheses. The lower limits on $m_{\mathrm{h}}$ and $m_{\mathrm{A}}$ in the no mixing (2 $\mathrm{TeV}$ ) scenario are only valid for $m_{\mathrm{A}}>2 \mathrm{GeV}$. 


\section{OPAL}
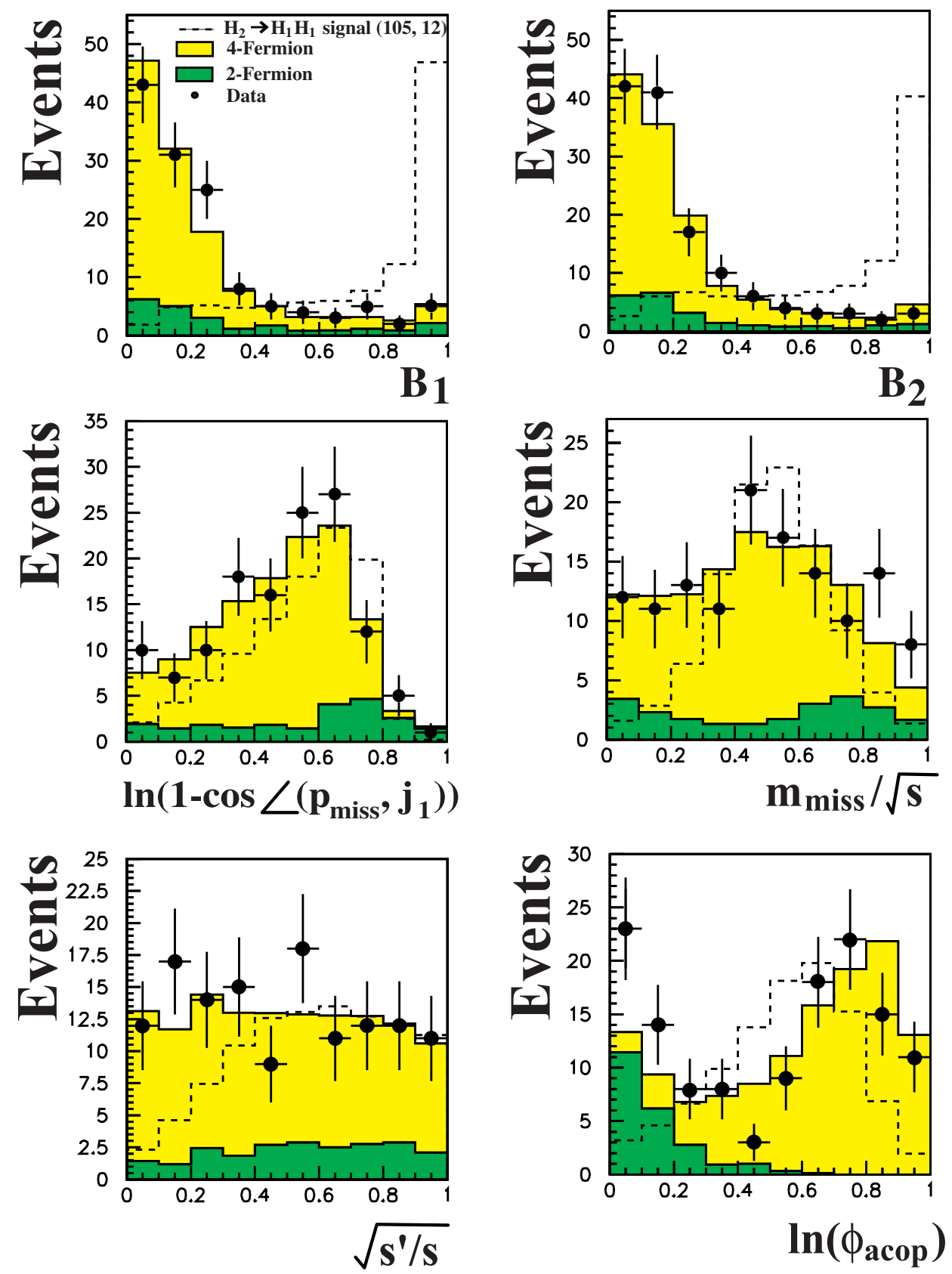

Figure 1: Distributions of some $A N N$ input variables for subsample $A$ for $\mathrm{e}^{+} \mathrm{e}^{-} \rightarrow \mathcal{H}_{2} \mathrm{Z} \rightarrow \mathrm{b} \overline{\mathrm{b}} \nu \bar{\nu}$ and $\mathrm{e}^{+} \mathrm{e}^{-} \rightarrow \mathcal{H}_{1} \mathcal{H}_{1} \mathrm{Z} \rightarrow \mathrm{b} \bar{b} b \bar{b} \nu \bar{\nu}$ (see Section 4.1.2) in the region $100 \mathrm{GeV}<m_{\mathcal{H}_{2}}<110 \mathrm{GeV}$ for the data taken at $\sqrt{s}=199-209 \mathrm{GeV}$. Light grey (yellow) is the four-fermion background, dark grey (green) the contribution from the $\mathrm{q} \overline{\mathrm{q}}(\gamma)$ background. The dashed line shows the arbitrarily scaled signal expectation for $m_{\mathcal{H}_{1}}=12 \mathrm{GeV}$ and $m_{\mathcal{H}_{2}}=105 \mathrm{GeV}$, as given in the legend. The points with error bars are the data. 
OPAL
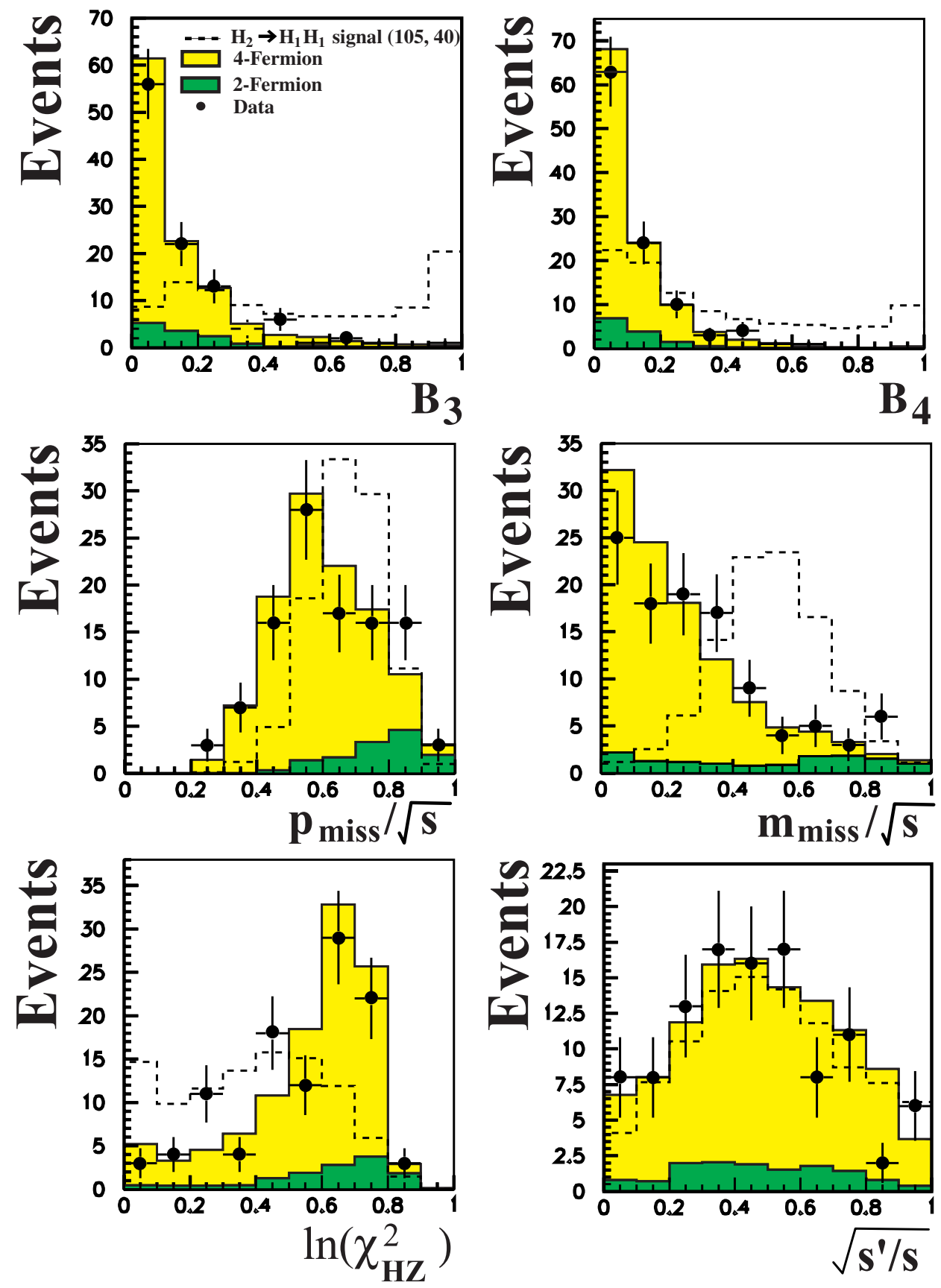

Figure 2: Distributions of some $A N N$ input variables for subsample $B$ for $\mathrm{e}^{+} \mathrm{e}^{-} \rightarrow \mathcal{H}_{2} \mathrm{Z} \rightarrow \mathrm{b} \overline{\mathrm{b}} \nu \bar{\nu}$ and $\mathrm{e}^{+} \mathrm{e}^{-} \rightarrow \mathcal{H}_{1} \mathcal{H}_{1} \mathrm{Z} \rightarrow \mathrm{b} \bar{b} b \bar{b} \nu \bar{\nu}$ (see Section 4.1.2) in the region $100 \mathrm{GeV}<m_{\mathcal{H}_{2}}<110 \mathrm{GeV}$. 


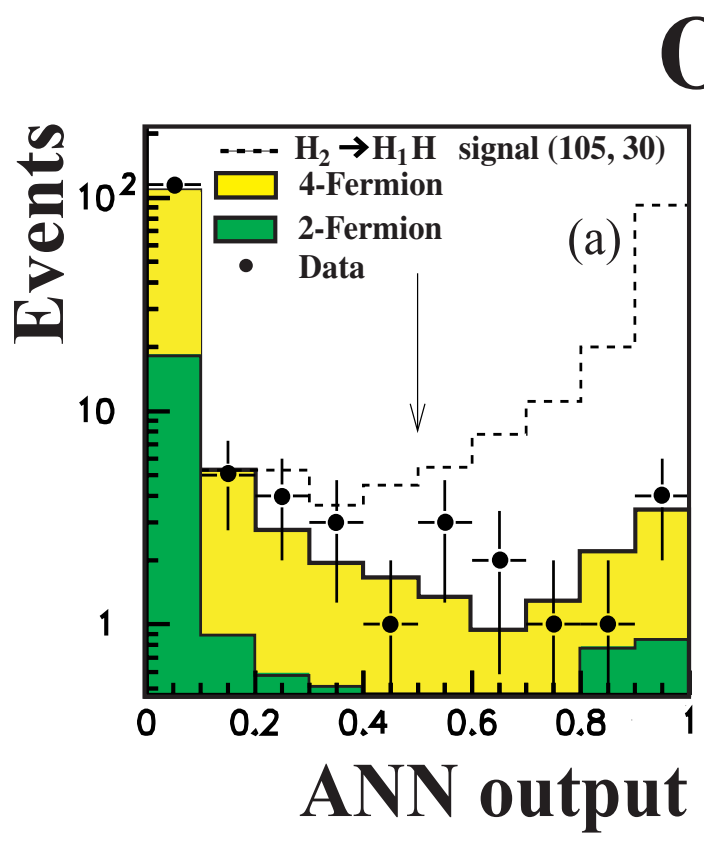

OPAL

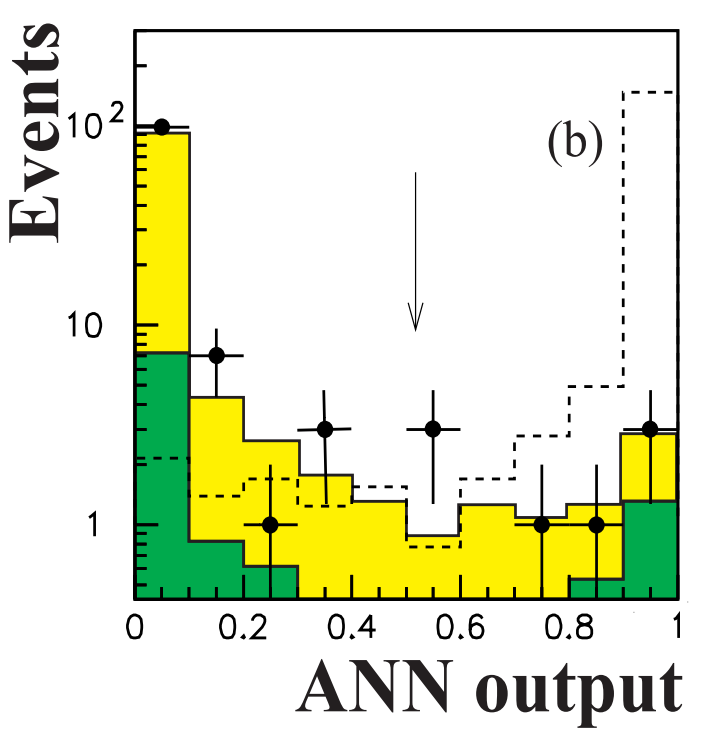

Figure 3: Distributions of $A N N_{\mathrm{A}}$ (a) and $A N N_{\mathrm{B}}$ (b) in the dedicated selection for $\mathrm{e}^{+} \mathrm{e}^{-} \rightarrow \mathcal{H}_{2} \mathrm{Z} \rightarrow \mathrm{b} \overline{\mathrm{b}} \nu \bar{\nu}$ and $\mathrm{e}^{+} \mathrm{e}^{-} \rightarrow \mathcal{H}_{1} \mathcal{H}_{1} \mathrm{Z} \rightarrow \mathrm{b} \bar{b} b \bar{b} \nu \bar{\nu}$ (see Section 4.1.2) in the region $100 \mathrm{GeV}<m_{\mathcal{H}_{2}}<110 \mathrm{GeV}$. The arrows indicate the cut on the ANN output value.

\section{OPAL}
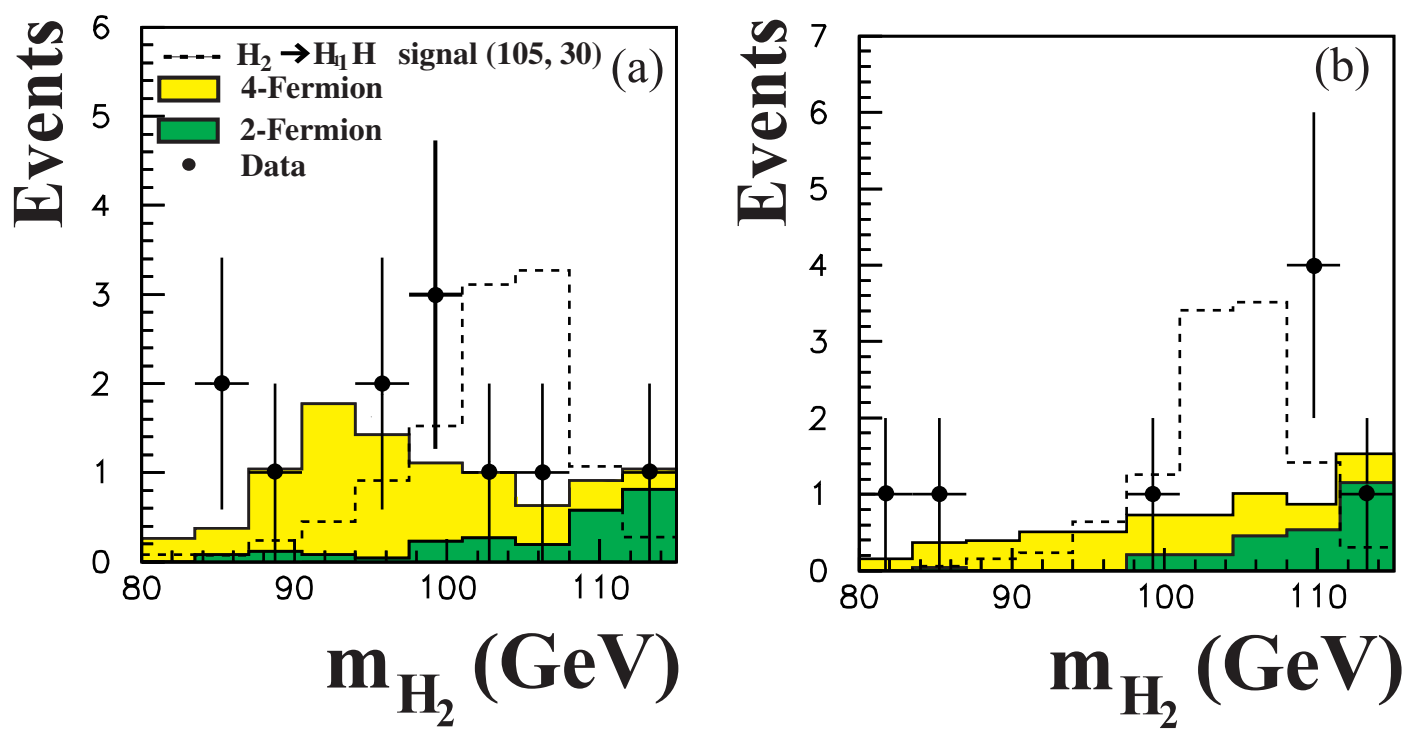

Figure 4: Distributions of the reconstructed masses in the selection $A$ and $B$ of the missing energy channel for data taken at $\sqrt{s}=199$ to $209 \mathrm{GeV}$ in the region $100 \mathrm{GeV}<m_{\mathcal{H}_{2}}<110$ $\mathrm{GeV}$ (see Section 4.1.2). 


\section{OPAL}
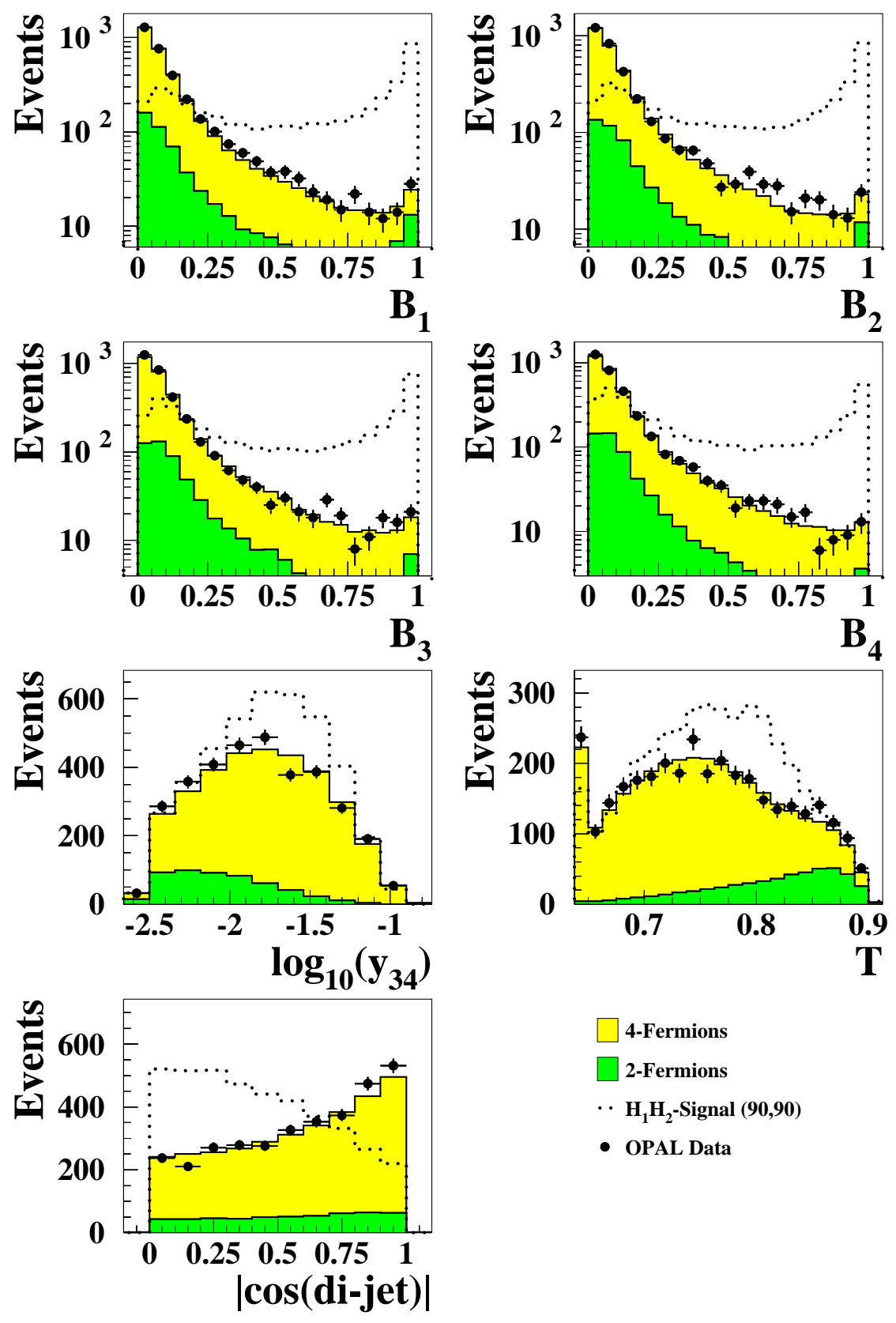

$\square$ 4-Fermions

$\square$ 2-Fermions

- $\mathbf{H}_{1} \mathbf{H}_{2}$-Signal $(90,90)$

- OPAL Data

Figure 5: Distributions of the likelihood input variables for the $\mathrm{e}^{+} \mathrm{e}^{-} \rightarrow \mathcal{H}_{1} \mathcal{H}_{2} \rightarrow \mathrm{b} \bar{b} b \bar{b}$ searches for high $m_{\mathcal{H}_{1}}$ at $192-209 \mathrm{GeV}$ (see Section 4.2.1). 
OPAL
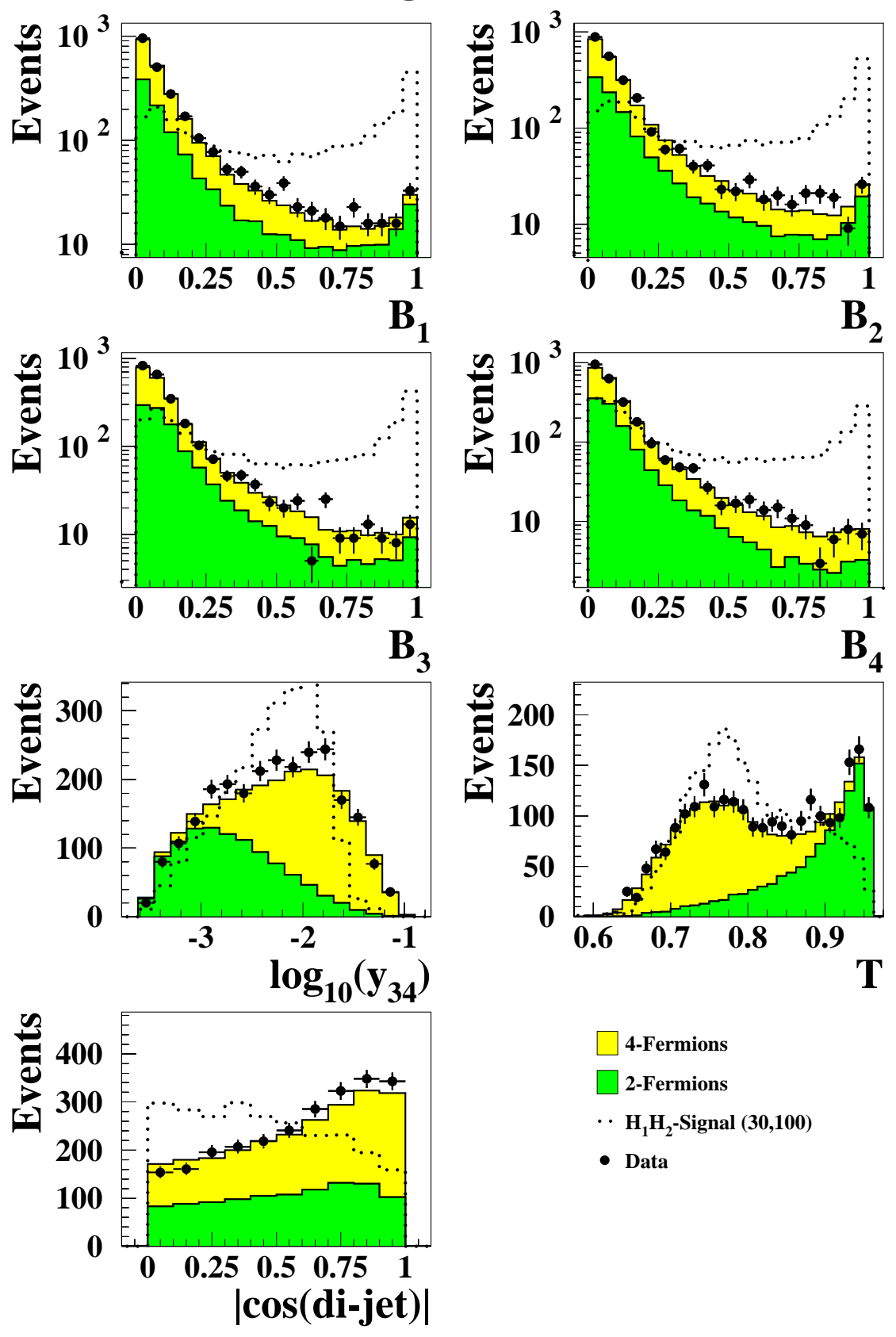

$\square$ 4-Fermions

$\square$ 2-Fermions

- $\mathbf{H}_{1} \mathrm{H}_{2}$-Signal (30,100)

- Data

Figure 6: Distributions of the input variables for the $\mathrm{e}^{+} \mathrm{e}^{-} \rightarrow \mathcal{H}_{1} \mathcal{H}_{2} \rightarrow \mathrm{b} \overline{\mathrm{b}} \overline{\mathrm{b}}$ searches for low $m_{\mathcal{H}_{1}}$ at 199-209 GeV (see Section 4.2.2). 

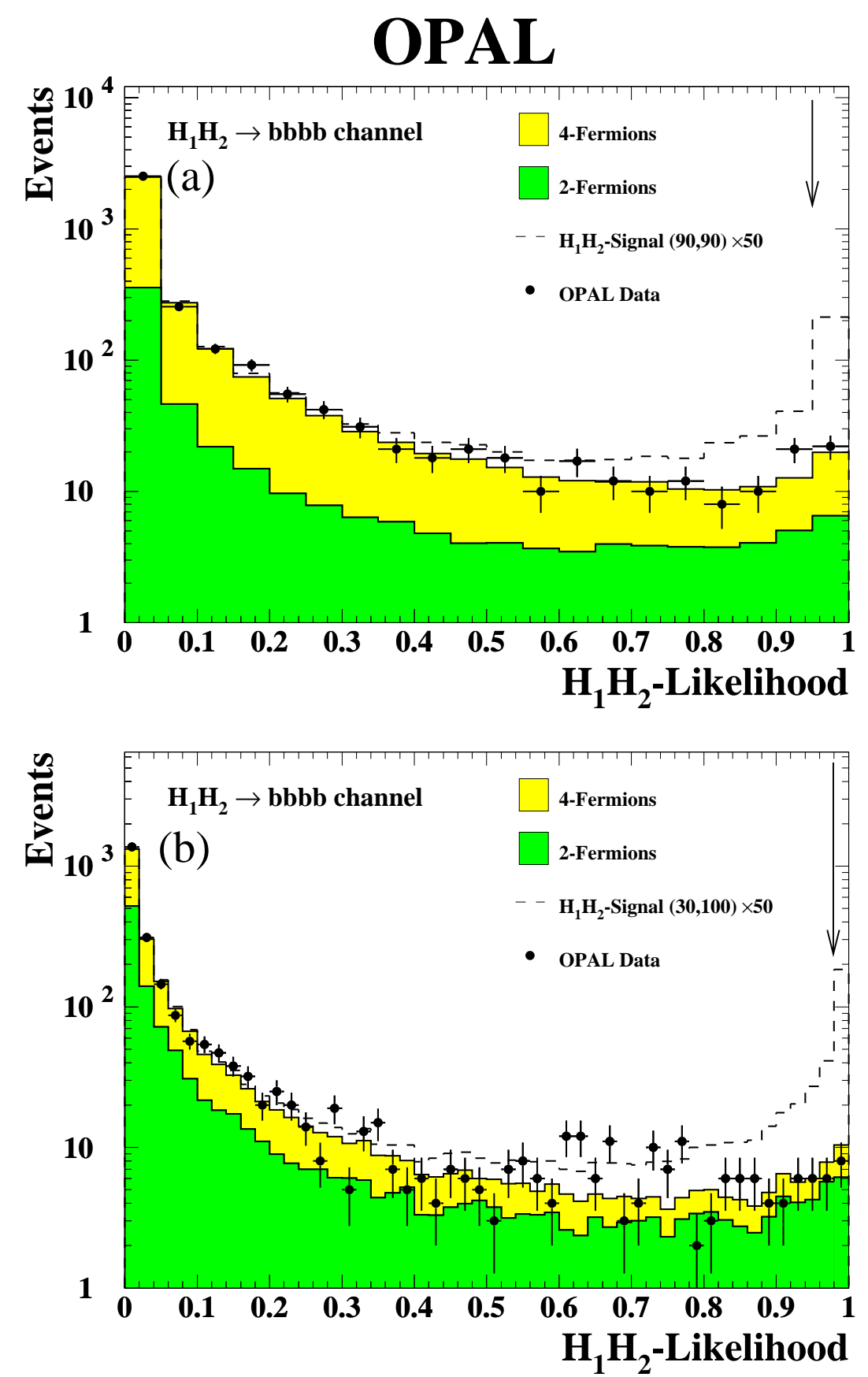

Figure 7: Searches for $\mathrm{e}^{+} \mathrm{e}^{-} \rightarrow \mathcal{H}_{1} \mathcal{H}_{2} \rightarrow \mathrm{b} \bar{b} b \bar{b}$ with high $m_{\mathcal{H}_{1}}$ (see Section 4.2.1, 4.2.2) at 192$209 \mathrm{GeV}$. Likelihood outputs for a) high $m_{\mathcal{H}_{1}}$, and b) low $m_{\mathcal{H}_{1}}$. The arrow indicates the cut position. The signal is scaled with a factor of 50 with respect to a $\mathrm{e}^{+} \mathrm{e}^{-} \rightarrow \mathcal{H}_{1} \mathcal{H}_{2} \rightarrow \mathrm{b} \bar{b} b \bar{b}$ signal for $\cos ^{2}(\beta-\alpha)=1$. 


\section{OPAL}

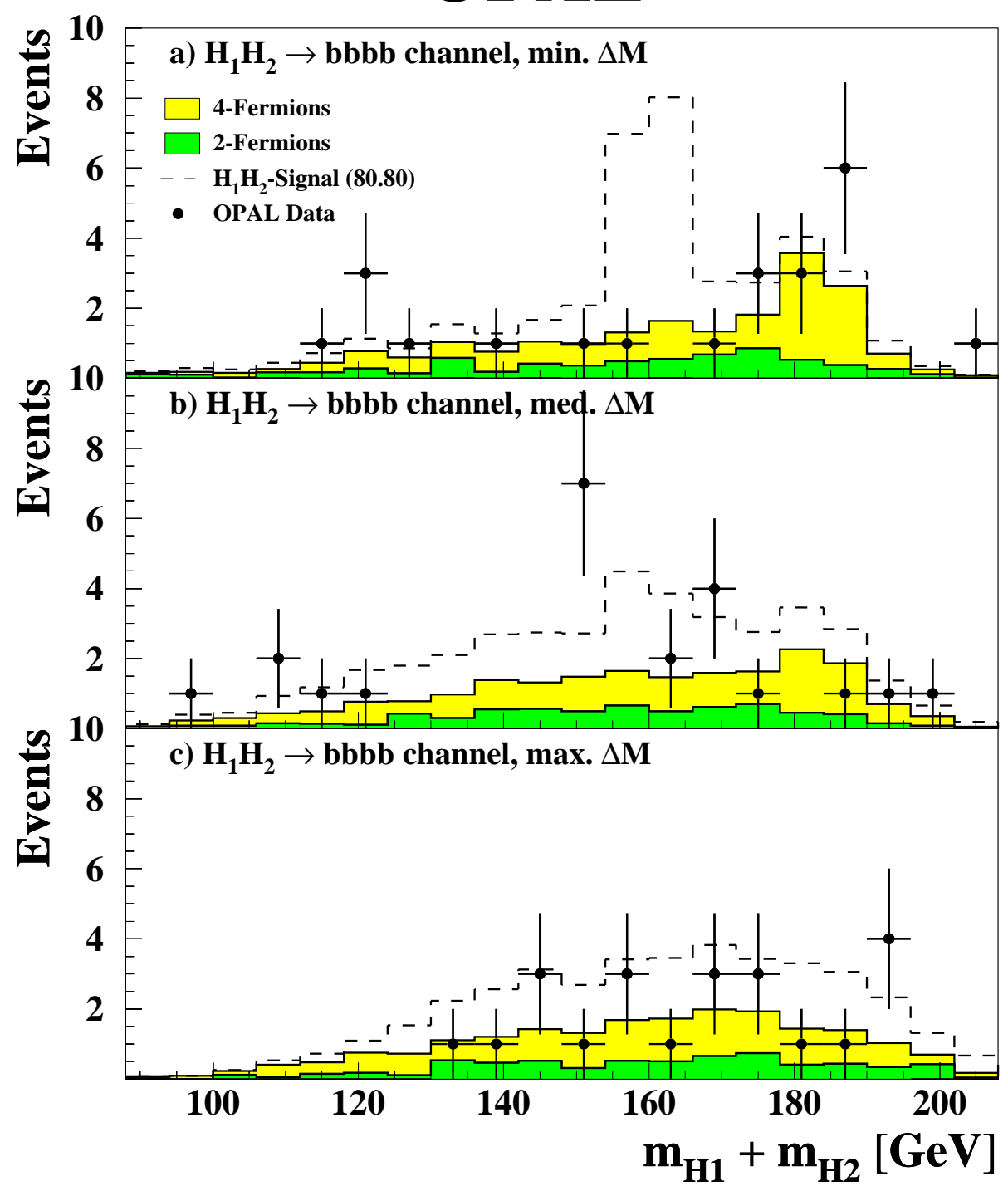

Figure 8: Searches for $\mathrm{e}^{+} \mathrm{e}^{-} \rightarrow \mathcal{H}_{1} \mathcal{H}_{2}$ with high $m_{\mathcal{H}_{1}}$ at $192-209 \mathrm{GeV}$. The sum of the reconstructed Higgs boson masses, $m_{\mathcal{H}_{1}}^{\mathrm{rec}}+m_{\mathcal{H}_{2}}^{\mathrm{rec}}$, for (a) the $\mathcal{H}_{1} \mathcal{H}_{2} \rightarrow 4 b$ channel with the di-jet pairing combination which yields the smallest mass difference, $\Delta M \equiv\left|m_{\mathcal{H}_{2}}^{\mathrm{rec}}-m_{\mathcal{H}_{1}}^{\mathrm{rec}}\right|$, (b) the $\mathcal{H}_{1} \mathcal{H}_{2} \rightarrow 4 b$ channel with the intermediate $\Delta M$ combination, (c) the $\mathcal{H}_{1} \mathcal{H}_{2} \rightarrow 4 b$ channel with the largest $\triangle M$ combination. OPAL data are indicated by points with error bars, the four-fermion background by the light grey (yellow) histograms, and the twofermion background by the darker grey (green) histograms. Shown as dashed histograms are the contributions expected from a Higgs boson signal with full strength at $m_{\mathcal{H}_{1}}=$ $m_{\mathcal{H}_{2}}=80 \mathrm{GeV}$ for a luminosity of $207 \mathrm{pb}^{-1}$ taken at $\sqrt{s}=199-209 \mathrm{GeV}$, added to the background expectation for a luminosity of $424 \mathrm{pb}^{-1}$ and the cross-section for $\cos ^{2}(\beta-\alpha)=1$. 


\section{OPAL}

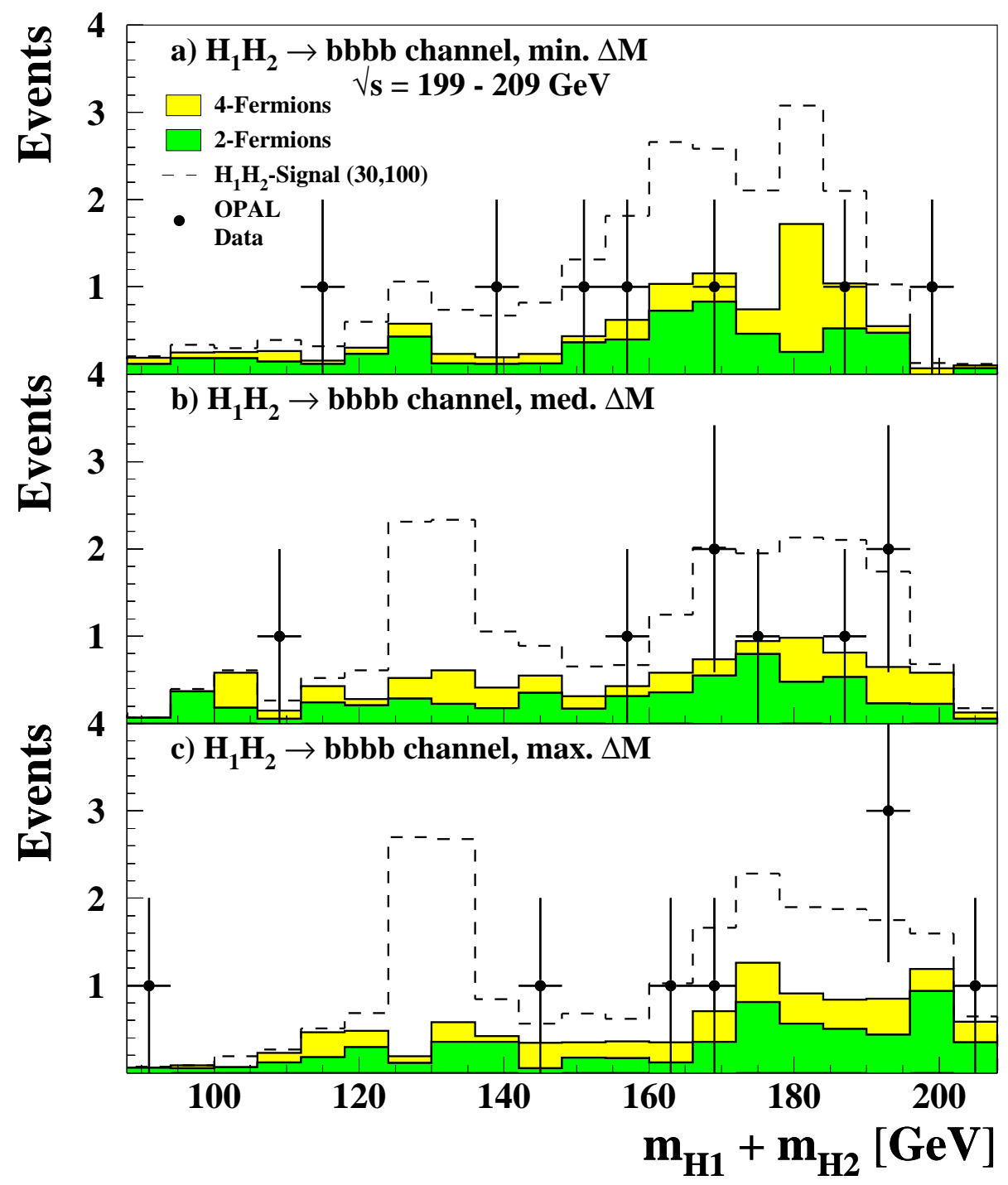

Figure 9: Searches for $\mathrm{e}^{+} \mathrm{e}^{-} \rightarrow \mathcal{H}_{1} \mathcal{H}_{2}$ with low $m_{\mathcal{H}_{1}}$ at $199-209 \mathrm{GeV}$. The sum of the reconstructed Higgs boson masses, $m_{\mathcal{H}_{1}}^{\mathrm{rec}}+m_{\mathcal{H}_{2}}^{\mathrm{rec}}$, for (a) the $\mathcal{H}_{1} \mathcal{H}_{2} \rightarrow 4 b$ channel with the dijet pairing combination which yields the smallest mass difference, $\Delta M \equiv\left|m_{\mathcal{H}_{2}}^{\mathrm{rec}}-m_{\mathcal{H}_{1}}^{\mathrm{rec}}\right|$, (b) the $\mathcal{H}_{1} \mathcal{H}_{2} \rightarrow 4 b$ channel with the medium $\Delta M$ combination, (c) the $\mathcal{H}_{1} \mathcal{H}_{2} \rightarrow 4 b$ channel with the maximum $\triangle M$ combination. OPAL data are indicated by points with error bars, the four-fermion background by the light grey (yellow) histograms, and the two-fermion background by the darker grey (green) histograms. Shown as dashed histograms are the contributions expected from a Higgs boson signal with $m_{\mathcal{H}_{1}}=30 \mathrm{GeV}, m_{\mathcal{H}_{2}}=100 \mathrm{GeV}$, added to the background expectation for a luminosity of $207 \mathrm{pb}^{-1}$ and the cross-section for $\cos ^{2}(\beta-\alpha)=1$. 

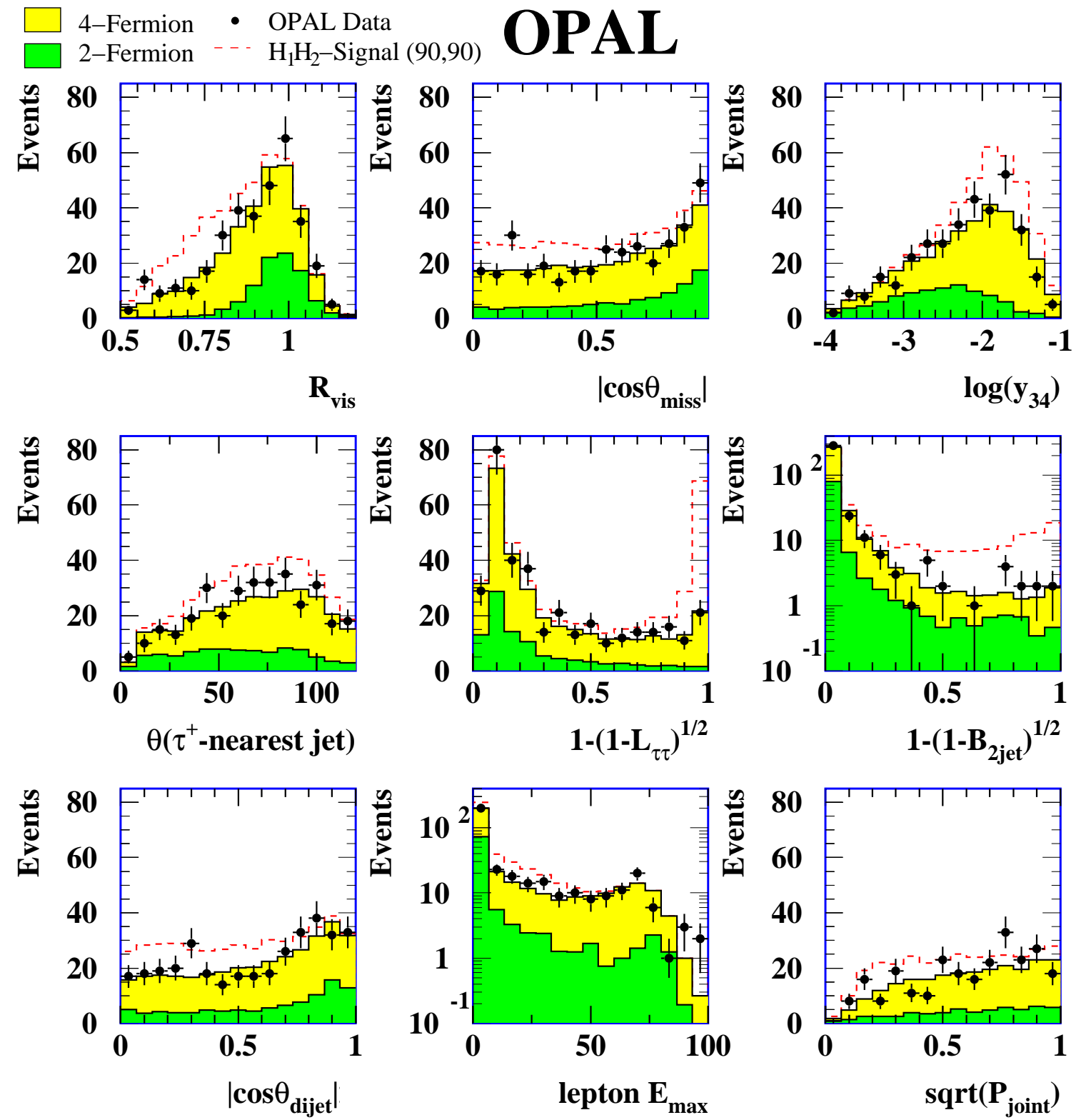

Figure 10: Input variables [7] for the $\mathrm{e}^{+} \mathrm{e}^{-} \rightarrow \mathcal{H}_{1} \mathcal{H}_{2} \rightarrow \mathrm{b} \overline{\mathrm{b}} \tau^{+} \tau^{-}$searches at 192-209 GeV. 


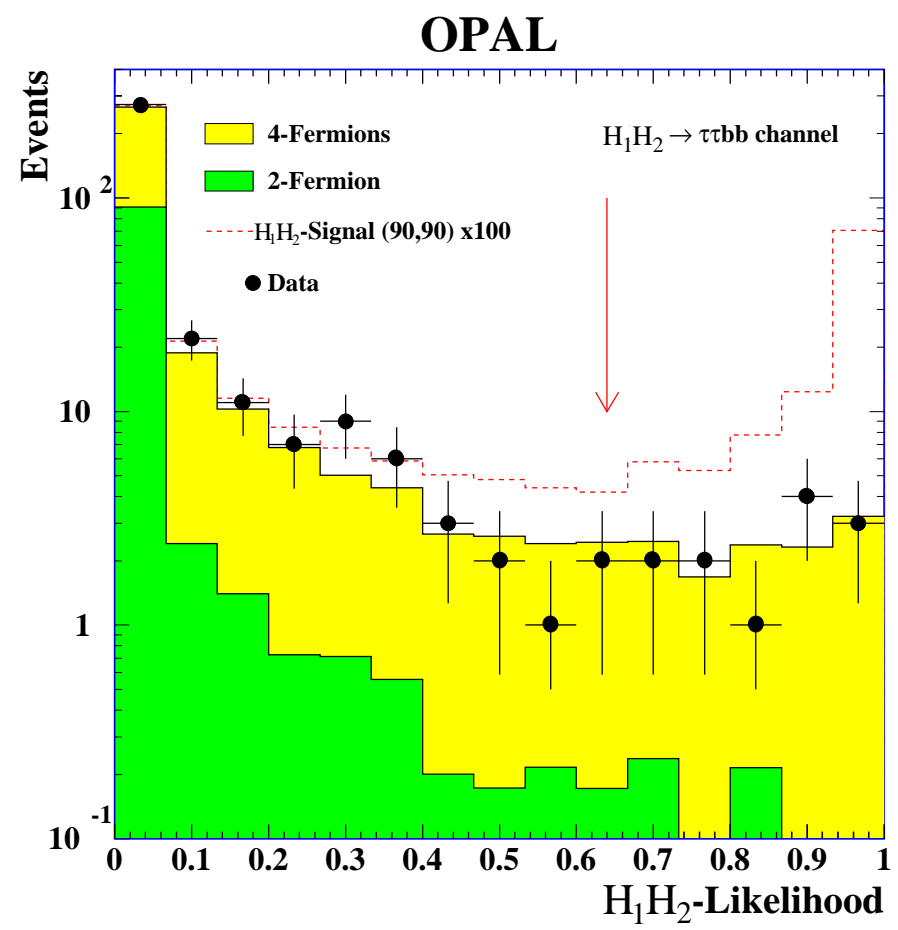

Figure 11: Likelihood output of the $\mathrm{e}^{+} \mathrm{e}^{-} \rightarrow \mathcal{H}_{1} \mathcal{H}_{2} \rightarrow \mathrm{b} \overline{\mathrm{b}} \tau^{+} \tau^{-}$search at $192-209 \mathrm{GeV}$. The arrow indicates the cut position. The signal is scaled with a factor of 100 with respect to a $\mathrm{e}^{+} \mathrm{e}^{-} \rightarrow \mathcal{H}_{1} \mathcal{H}_{2} \rightarrow \mathrm{b} \overline{\mathrm{b}} \tau^{+} \tau^{-}$signal for $\cos ^{2}(\beta-\alpha)=1$. 

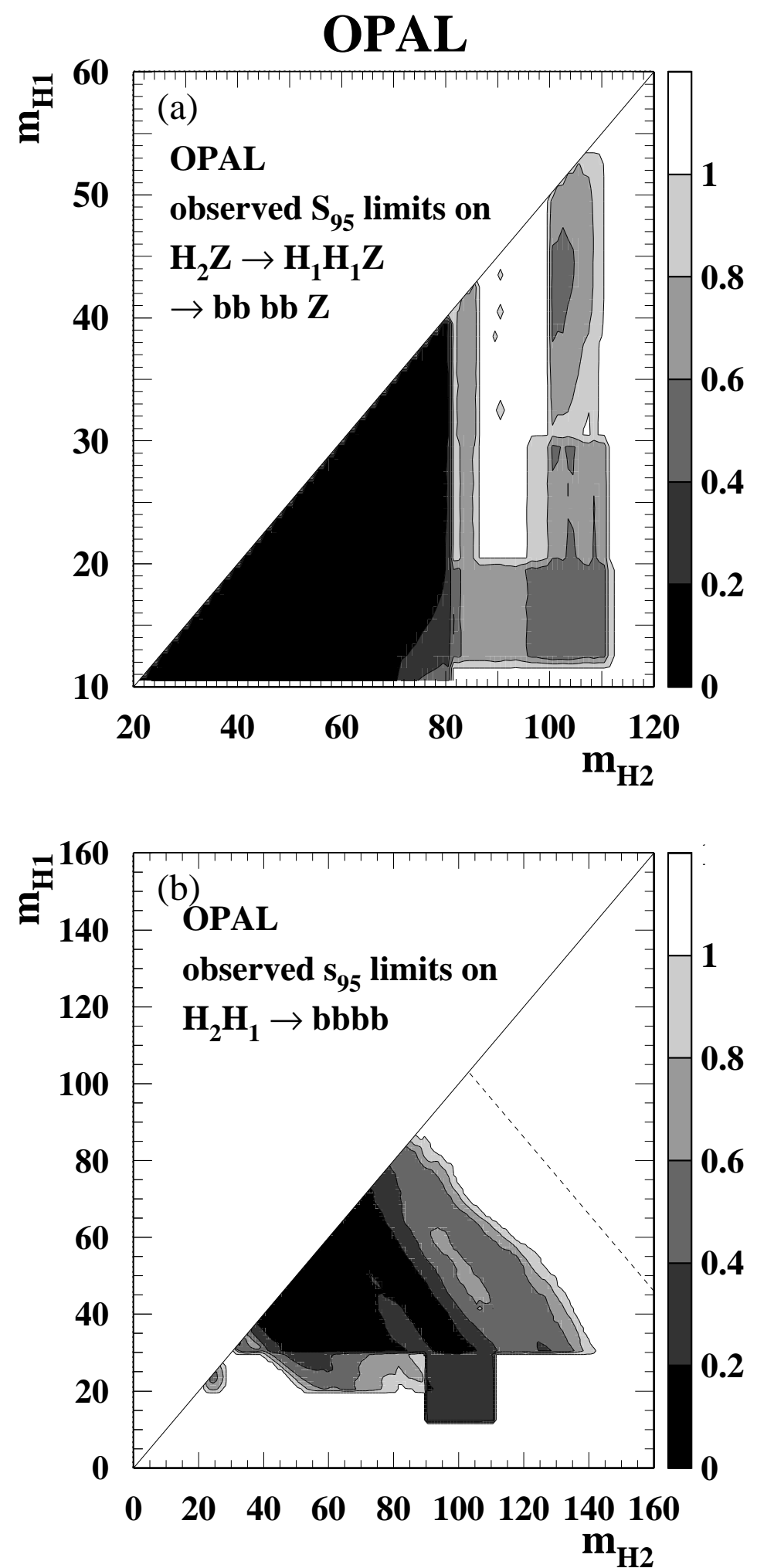

Figure 12: Model-independent upper bounds on $\sigma \times \mathrm{BR}$ for (a) the $\mathrm{e}^{+} \mathrm{e}^{-} \rightarrow \mathcal{H}_{2} \mathrm{Z} \rightarrow \mathcal{H}_{1} \mathcal{H}_{1} \mathrm{Z} \rightarrow \mathrm{bb} b \overline{b Z}$ channel and (b) the $\mathrm{e}^{+} \mathrm{e}^{-} \rightarrow \mathcal{H}_{1} \mathcal{H}_{2} \rightarrow \mathrm{bb} \bar{b}$ channel. For (a), the $S M$ cross-section for $\mathrm{H}_{\mathrm{SM}} \mathrm{Z}$ production is taken as normalization. For (b), The MSSM cross-section for $\mathcal{H}_{1} \mathcal{H}_{2}$ production with $\cos ^{2}(\beta-\alpha)=1$ is taken as normalization. The dashed line indicates the kinematic limit for $\sqrt{s}=206 \mathrm{GeV}$. 

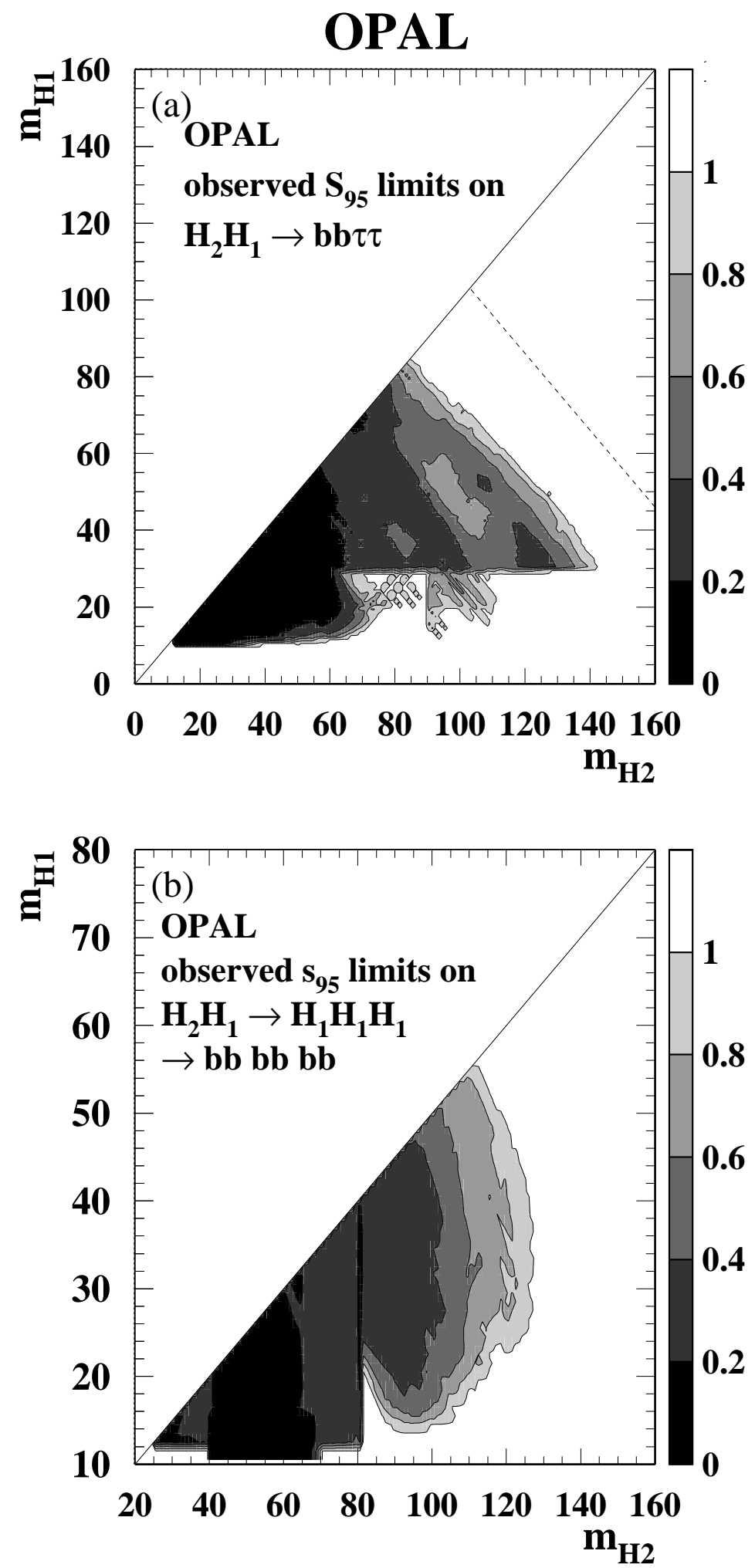

Figure 13: Model-independent upper bounds on $\sigma \times \mathrm{BR}$ for (a) the $\mathrm{e}^{+} \mathrm{e}^{-} \rightarrow \mathcal{H}_{1} \mathcal{H}_{2} \rightarrow \mathrm{b} \overline{\mathrm{b}} \tau^{+} \tau^{-}$ channel and (b) the $\mathrm{e}^{+} \mathrm{e}^{-} \rightarrow \mathcal{H}_{1} \mathcal{H}_{2} \rightarrow \mathcal{H}_{1} \mathcal{H}_{1} \mathcal{H}_{1} \rightarrow \mathrm{b} \bar{b} b \bar{b} b \bar{b}$ channel. The MSSM cross-section for $\mathcal{H}_{1} \mathcal{H}_{2}$ production with $\cos ^{2}(\beta-\alpha)=1$ is taken as normalisation. The dashed line indicates the kinematic limit for $\sqrt{s}=206 \mathrm{GeV}$. 

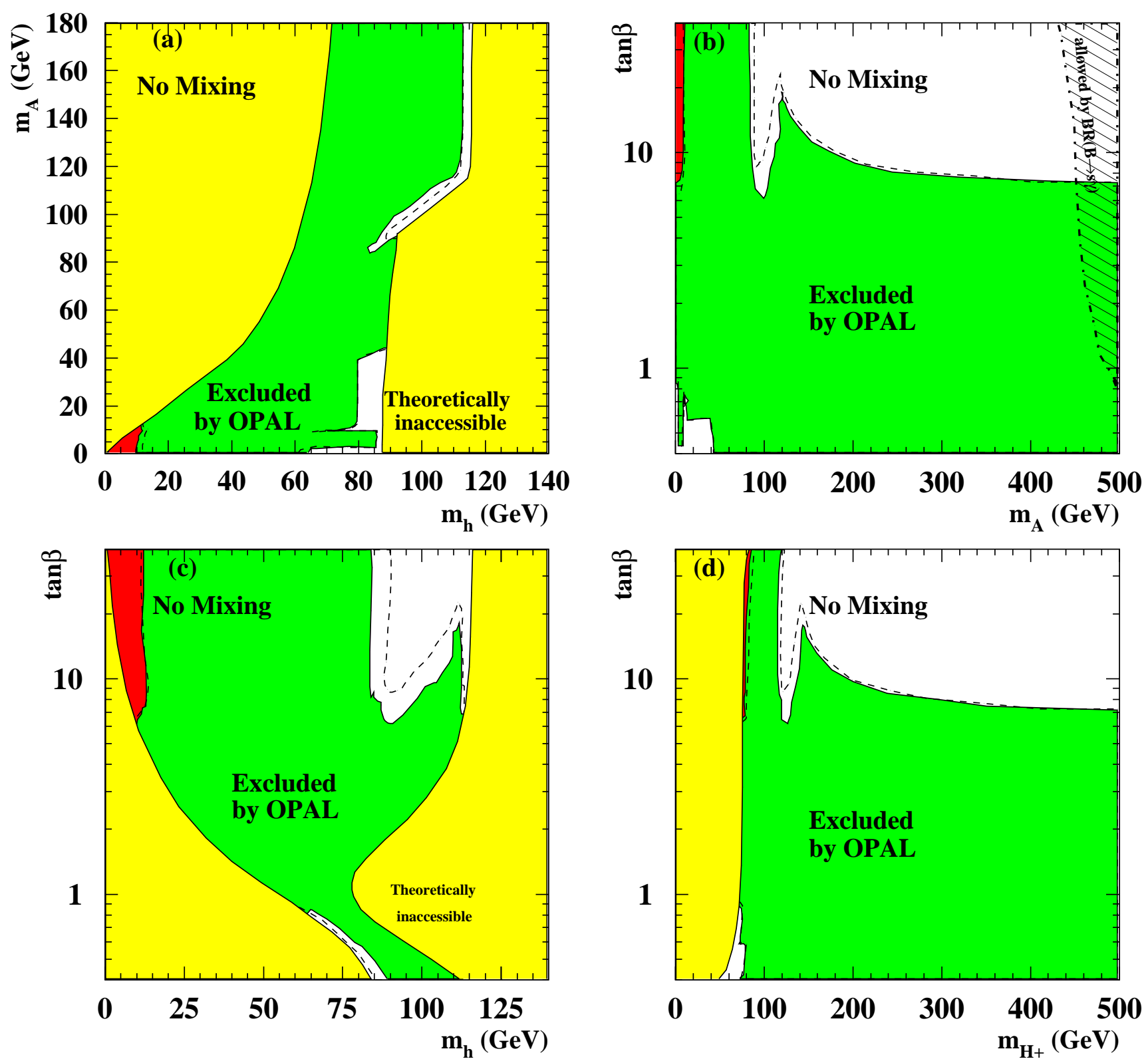

Figure 14: Results for the no mixing benchmark scenario. The figure shows the excluded regions in darker grey (green) and theoretically inaccessible regions in light grey (yellow) as functions of the MSSM parameters in four projections: (a) the $\left(m_{\mathrm{h}}, m_{\mathrm{A}}\right)$ plane, (b) the $\left(m_{\mathrm{A}}, \tan \beta\right)$ plane, (c) the $\left(m_{\mathrm{h}}, \tan \beta\right)$ plane and $(d)$ the $\left(m_{\mathrm{H}^{ \pm}}, \tan \beta\right)$ plane. The dashed lines indicate the boundaries of the regions expected to be excluded at the $95 \% \mathrm{CL}$ if only SM background processes are present. The region excluded by Yukawa searches, Z-width constraints or decay independent searches is shown in dark grey (red). In (b) the hatched area is still allowed by $\mathrm{BR}(\mathrm{b} \rightarrow \mathrm{s} \gamma)$ searches. 

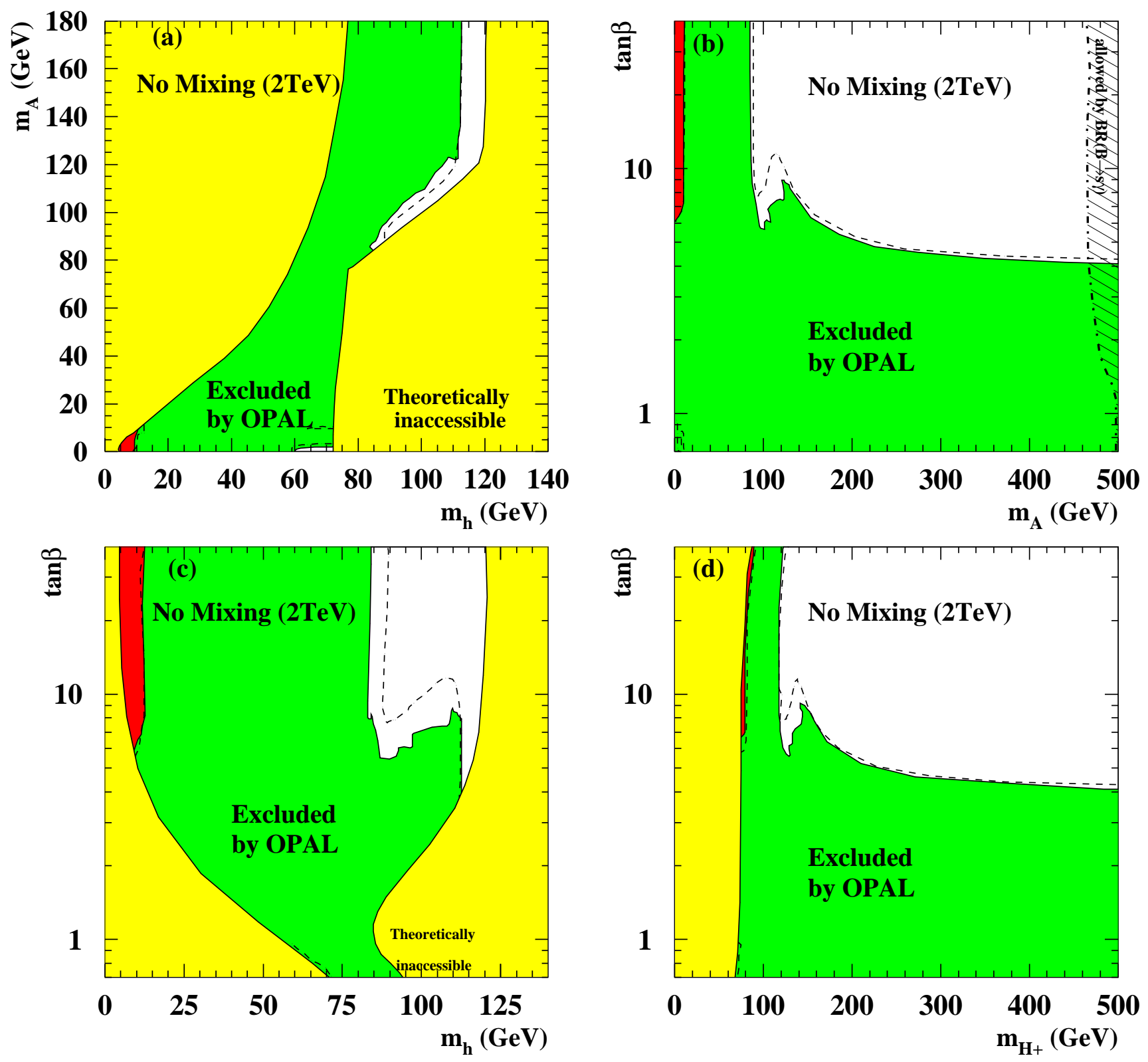

Figure 15: Results for the no mixing (2 TeV) benchmark scenario described in the text of Section 7.1. See Fig. 14 for the notation. 

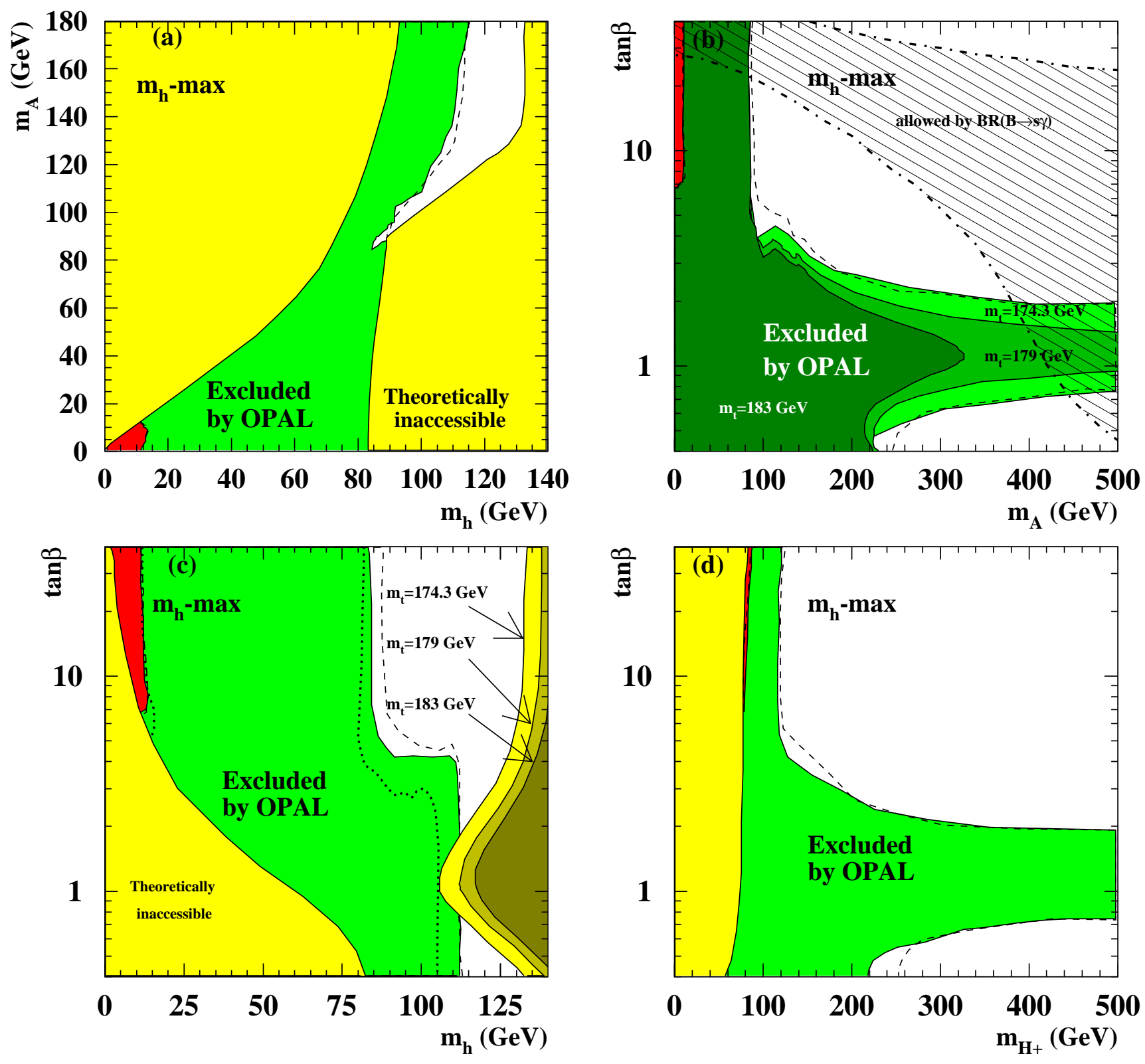

Figure 16: Results for the $m_{\mathrm{h}}-\max$ benchmark scenario. See Fig. 14 for the notation. The dotted line in (c) shows the observed 99.9\% confidence limit. The differently shaded regions in (b) show the exclusion for different values of $m_{\mathrm{top}}$, as written in the Plot. The upper limit on $m_{\mathrm{h}}$ for different values of $m_{\mathrm{top}}$ is shown in (c), as expressed in the plot. 

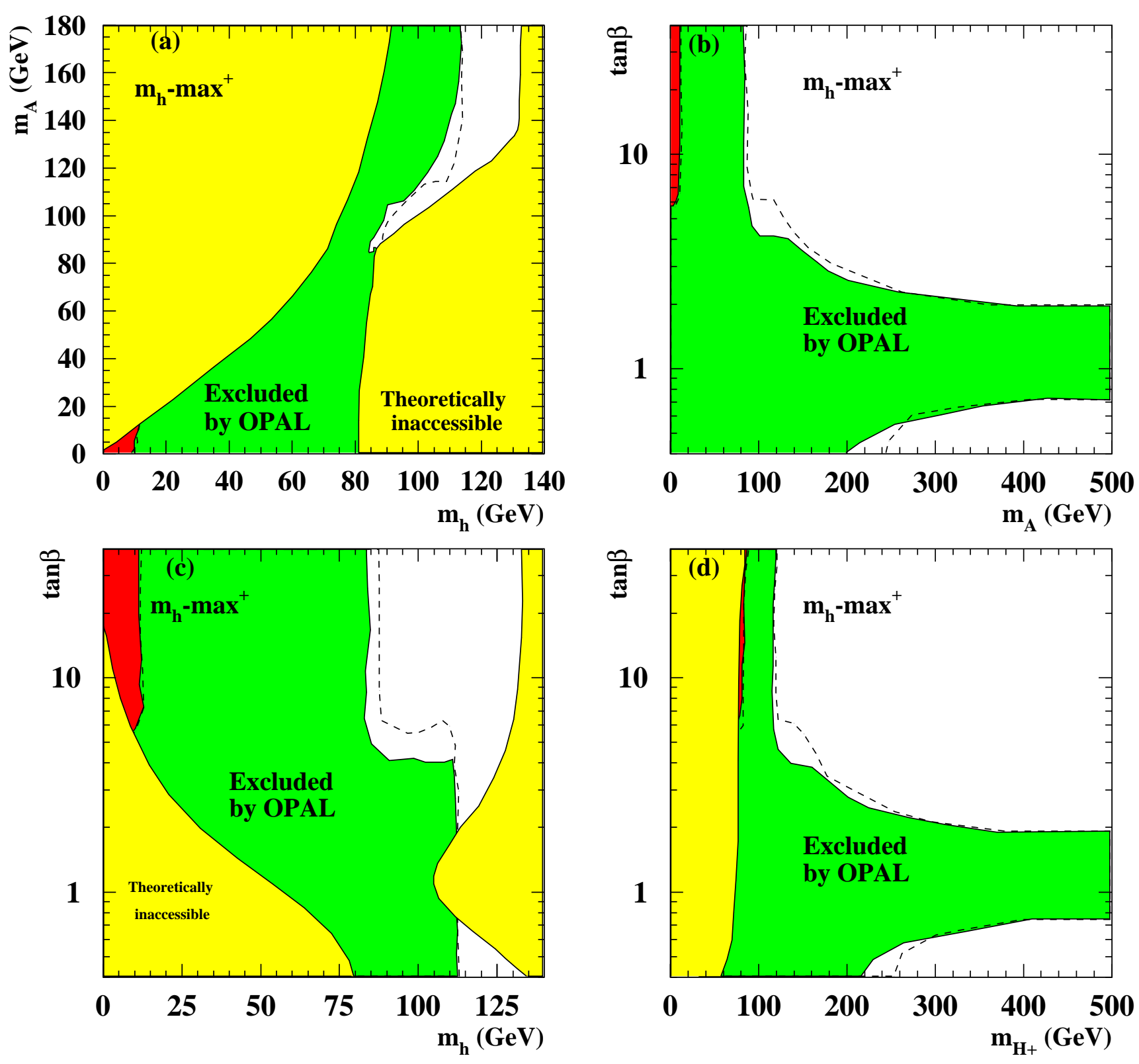

Figure 17: Results for the $m_{\mathrm{h}}-\max ^{+}$benchmark scenario described in the text of Section [7.1. See Fig. 14 for the notation. This scenario is excluded by the $B R(b \rightarrow s \gamma)$ constraint for $m_{\mathrm{A}}<600 \mathrm{GeV}$. 

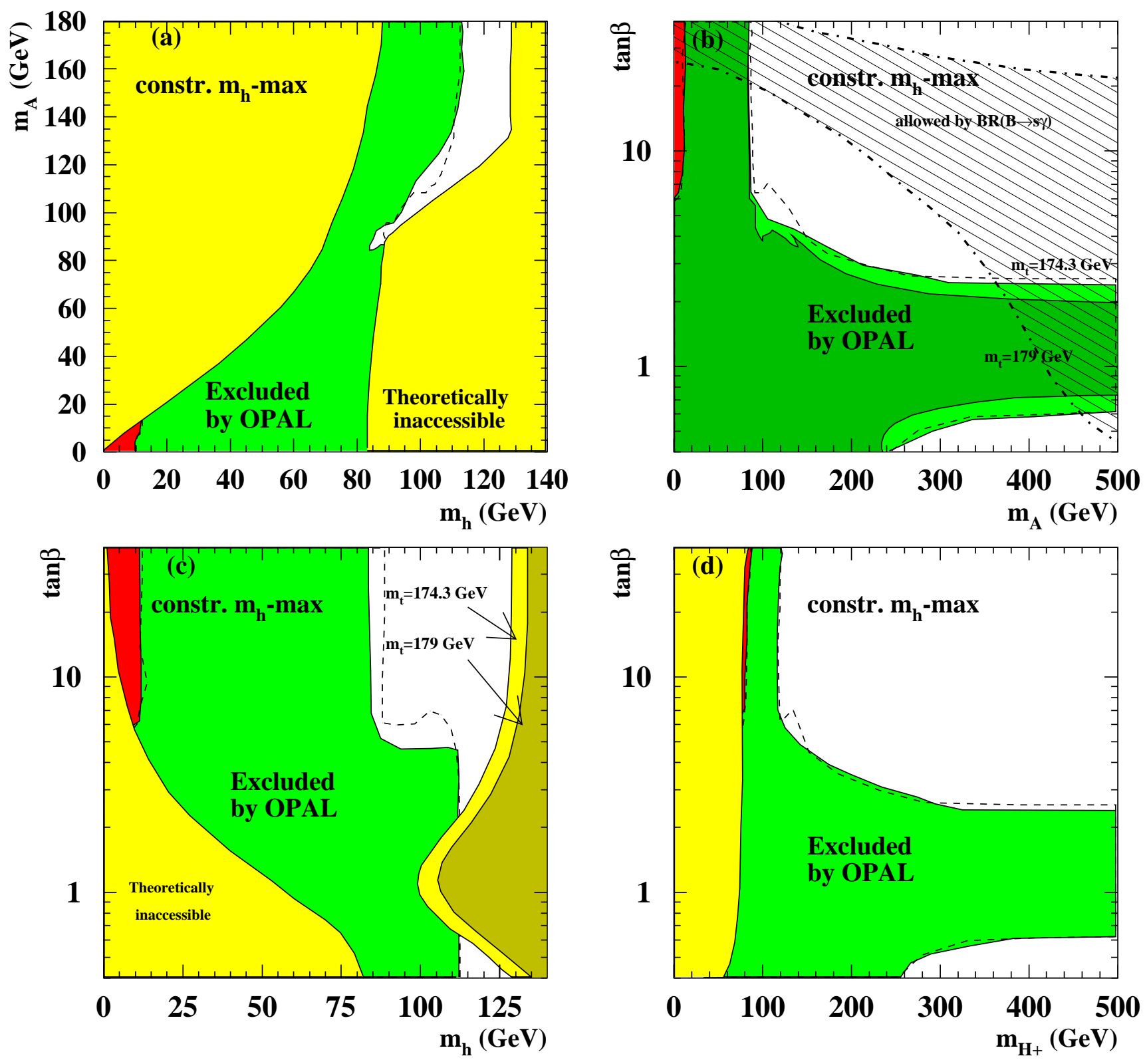

Figure 18: Results for the constrained $m_{\mathrm{h}}$ - max benchmark scenario described in the text of Section 7.1] See Fig. 14 for the notation. The differently shaded regions in (b) show the exclusion for different values of $m_{\mathrm{top}}$, as written in the Plot. The upper limit on $m_{\mathrm{h}}$ for different values of $m_{\mathrm{top}}$ is shown in (c), as also expressed in the plot. 

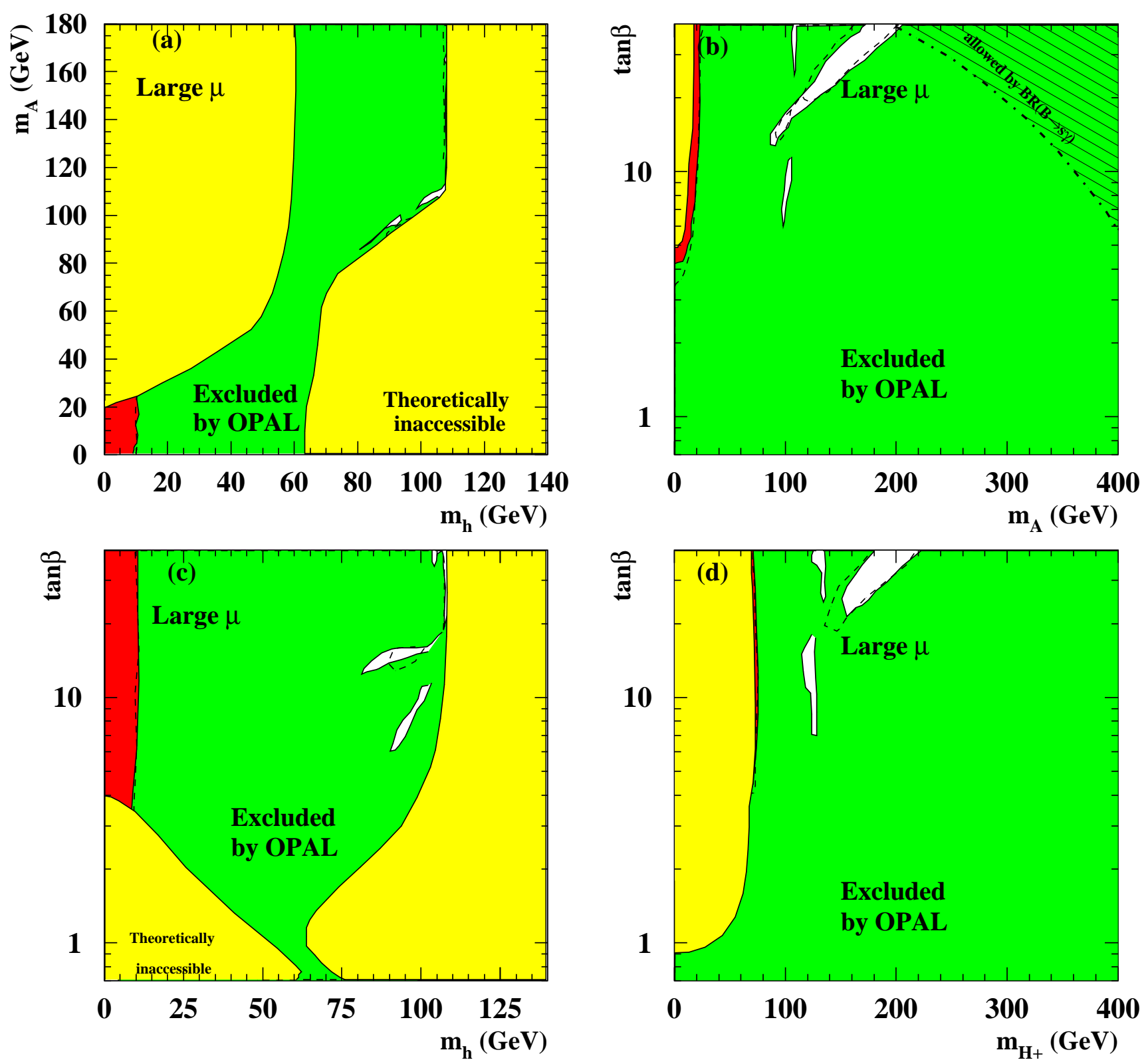

Figure 19: Results for the large $\mu$ benchmark scenario described in the text of Section 7.1, See Fig. 14 for the notation. 

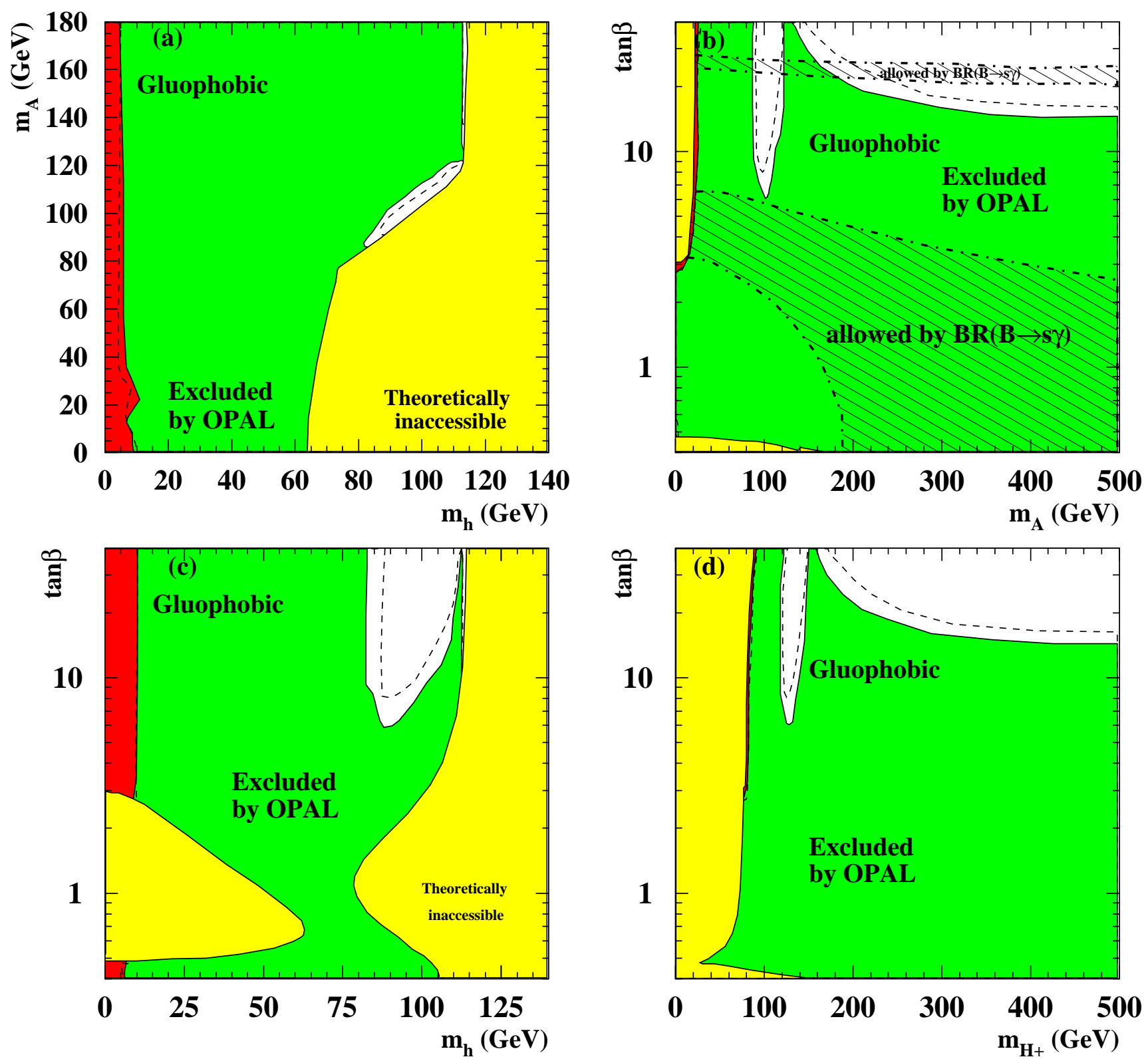

Figure 20: Results for the gluophobic benchmark scenario described in the text of Section [7.1. See Fig. 14 for the notation. The hatched area in (c) is allowed by the $B R(b \rightarrow s \gamma)$ constraint. 

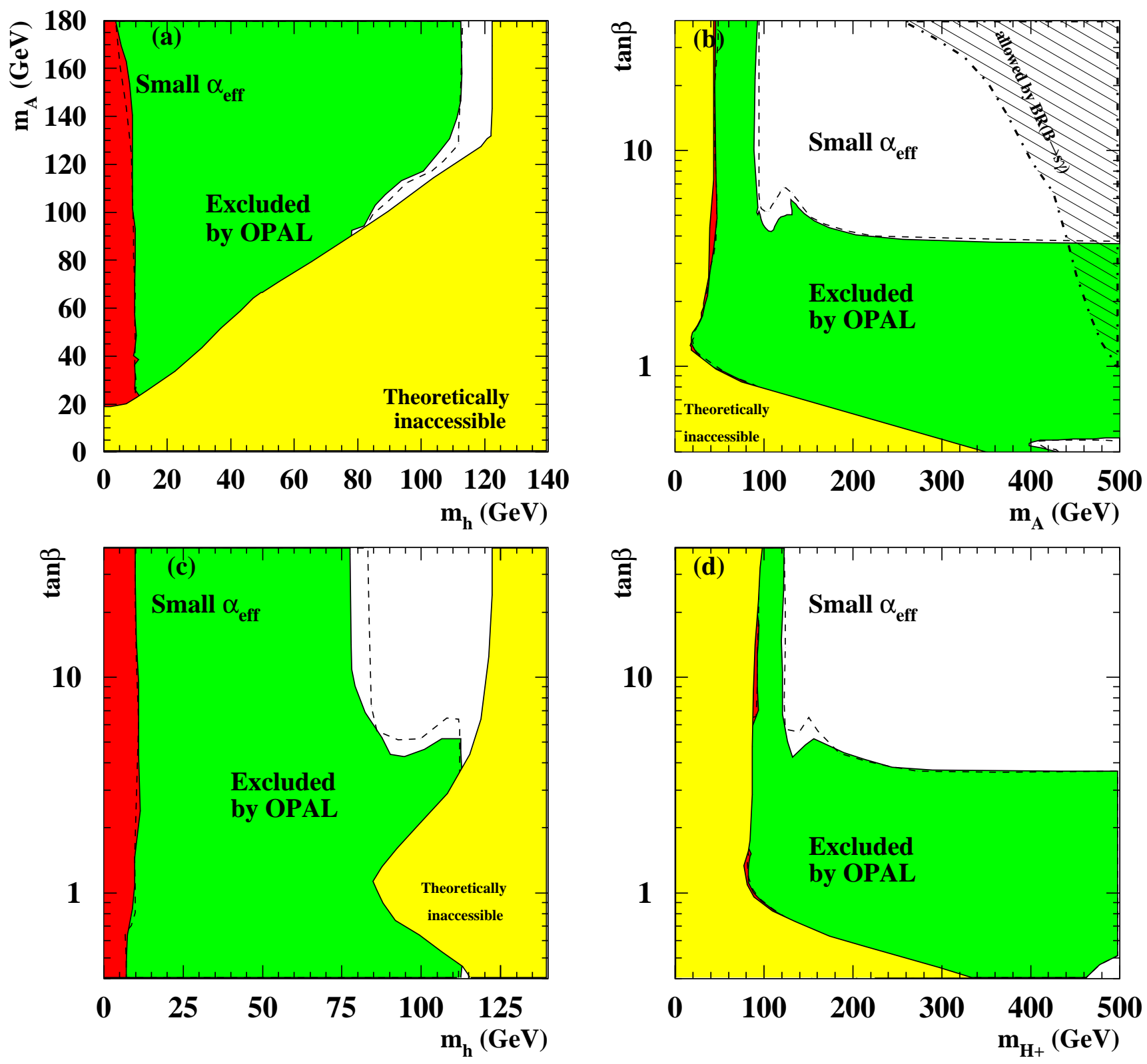

Figure 21: Results for the small $\alpha_{\mathrm{eff}}$ benchmark scenario described in the text of Section [7.1. See Fig. 14] for the notation. 


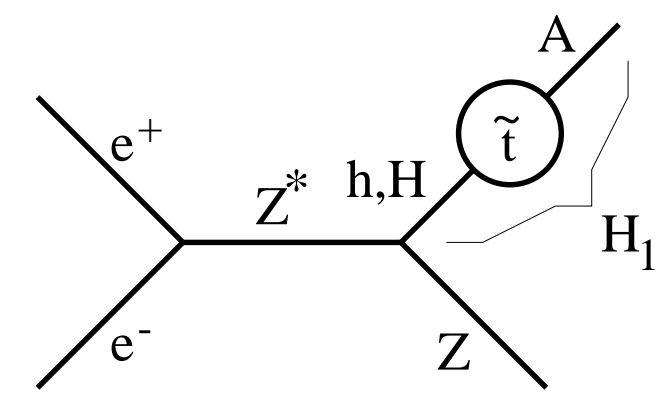

Figure 22: Diagram illustrating the effective coupling of a Higgs mass eigenstate to the $\mathrm{Z}$ in Higgsstrahlung. The complete mass eigenstate $\mathcal{H}_{1}$ is composed of admixtures of $\mathrm{h}, \mathrm{H}$ and $\mathrm{A}$. Here the $\mathrm{h}, \mathrm{H}$ and $\mathrm{A}$ denote the CP-even and CP-odd weak eigenstates, respectively. Only the $\mathrm{CP}$-even admixtures $\mathrm{h}$ and $\mathrm{H}$ couple to the $\mathrm{Z}$, while the $\mathrm{CP}$-odd $\mathrm{A}$ does not. Therefore the coupling of the mass eigenstate is reduced with respect to a CPC scenario. 

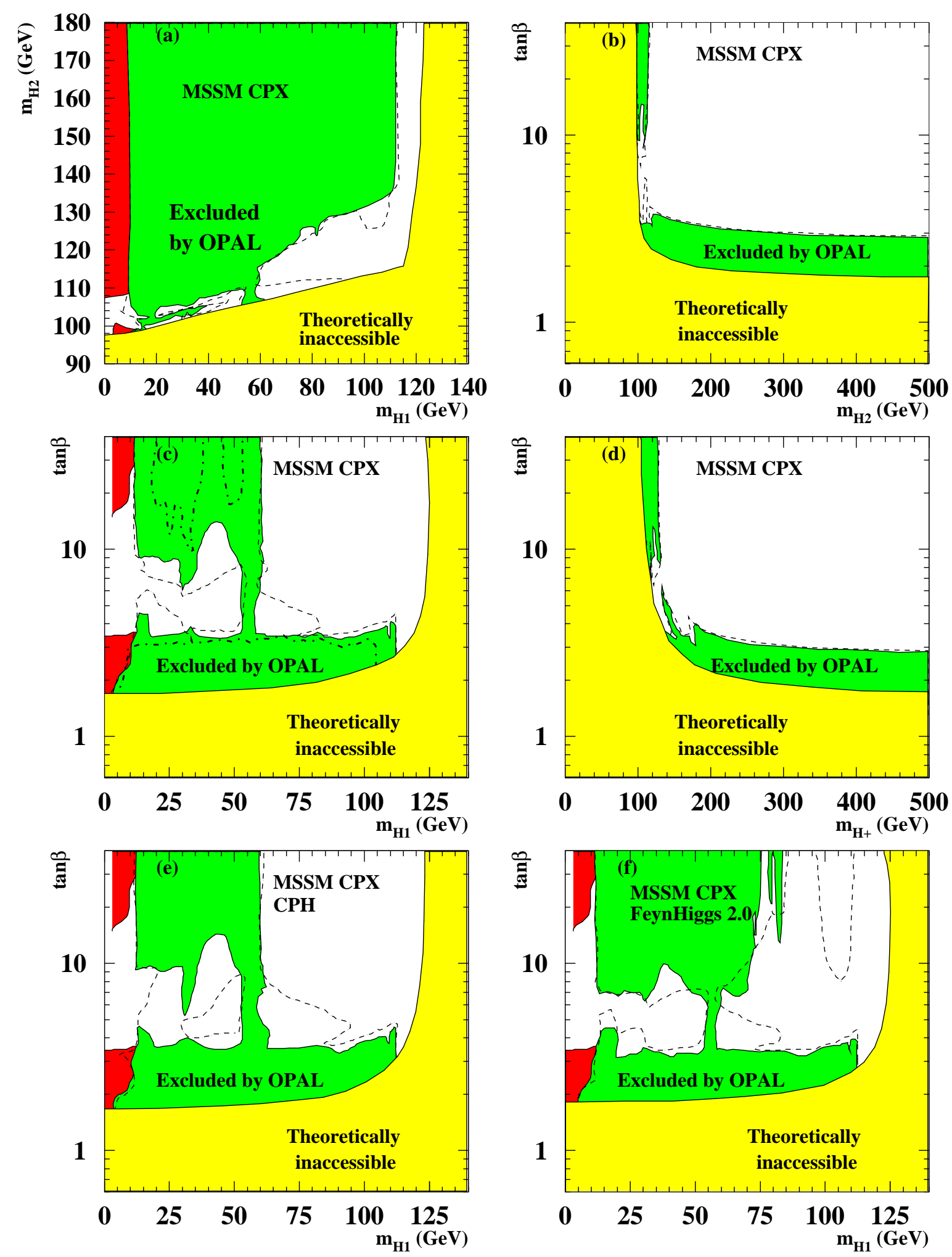

Figure 23: The CPX MSSM 95\% CL exclusion areas. Excluded regions are shown for (a) the $\left(m_{\mathrm{H}_{1}}, m_{\mathrm{H}_{2}}\right)$ plane, (b) the $\left(m_{\mathrm{H}_{2}}, \tan \beta\right)$ plane, (c) the $\left(m_{\mathrm{H}_{1}}, \tan \beta\right)$ plane and (d) the $\left(m_{\mathrm{H}^{ \pm}}, \tan \beta\right)$ plane. Figure (e) shows the $\left(m_{\mathrm{H}_{1}}, \tan \beta\right)$ of the $C P H$ calculation alone, (f) shows the same projection of the FEYNHIGGS 2.0 calculation. See Fig. 14 for the notation. The dash-dotted line in (c) shows the area excluded on the $99.9 \%$ confidence level. In (b) and (d) the area excluded by $\mathrm{Z}$ width constraints or by decay independent searches is too small to be displayed. 

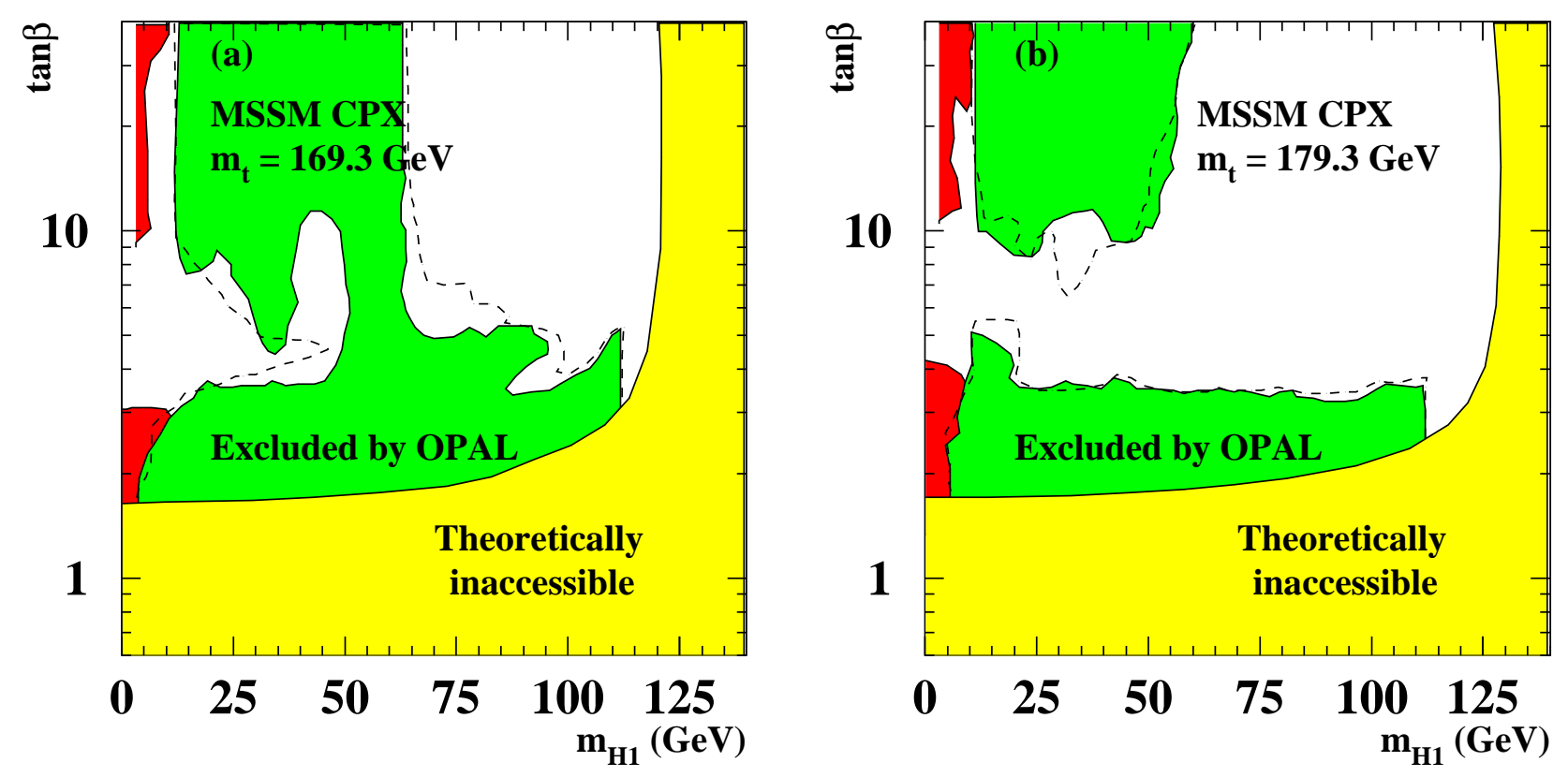

Figure 24: The CPX MSSM 95\% CL exclusion areas in the $\left(m_{\mathrm{H}_{1}}, \tan \beta\right)$ plane, using scans with (a) $m_{\mathrm{t}}=179.3 \mathrm{GeV}$ and (b) $m_{\mathrm{t}}=169.3 \mathrm{GeV}$. Due to the change in the top masses a strong difference is observed compared to Fig. 223 (c). See Fig. 114 for the notation. 

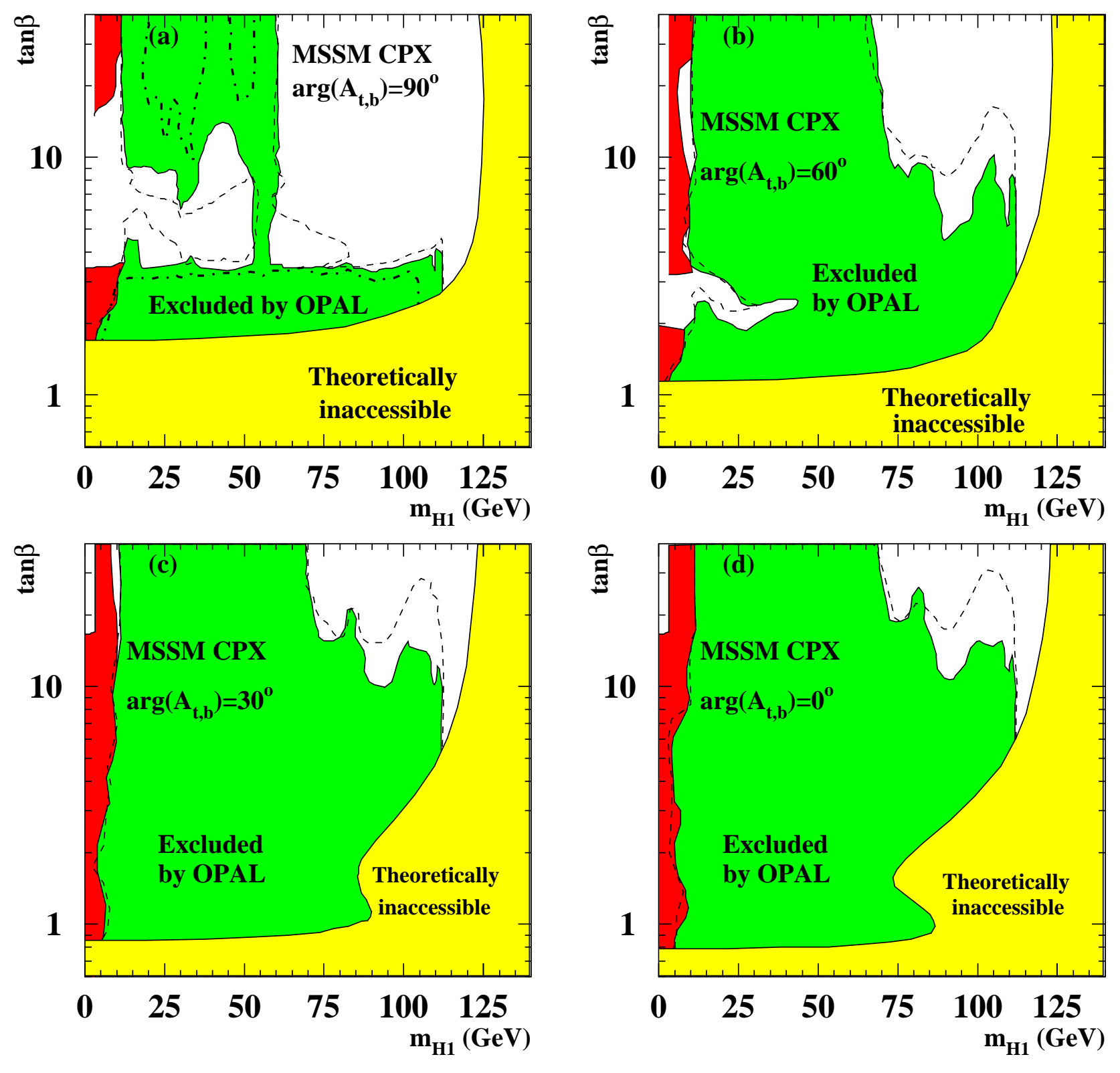

Figure 25: The CPX MSSM $95 \% \mathrm{CL}$ exclusion areas in the $\left(m_{\mathrm{H}_{1}}, \tan \beta\right)$ plane, using scans with (a) $\arg \left(A_{\mathrm{t}, \mathrm{b}}\right)=\arg \left(m_{\tilde{\mathrm{g}}}\right)=90^{\circ},(\mathrm{b}) \arg \left(A_{\mathrm{t}, \mathrm{b}}\right)=\arg \left(m_{\tilde{\mathrm{g}}}\right)=60^{\circ}$, (c) $\arg \left(A_{\mathrm{t}, \mathrm{b}}\right)=$ $\arg \left(m_{\tilde{\mathrm{g}}}\right)=30^{\circ}$ and $(\mathrm{d}) \arg \left(A_{\mathrm{t}, \mathrm{b}}\right)=\arg \left(m_{\tilde{\mathrm{g}}}\right)=0^{\circ}$. While the CPV phases decrease, effects from $C P$ violation like the strong $\mathrm{H}_{2} \rightarrow \mathrm{H}_{1} \mathrm{H}_{1}$ contribution vanish. See Fig. 14 for the notation. 

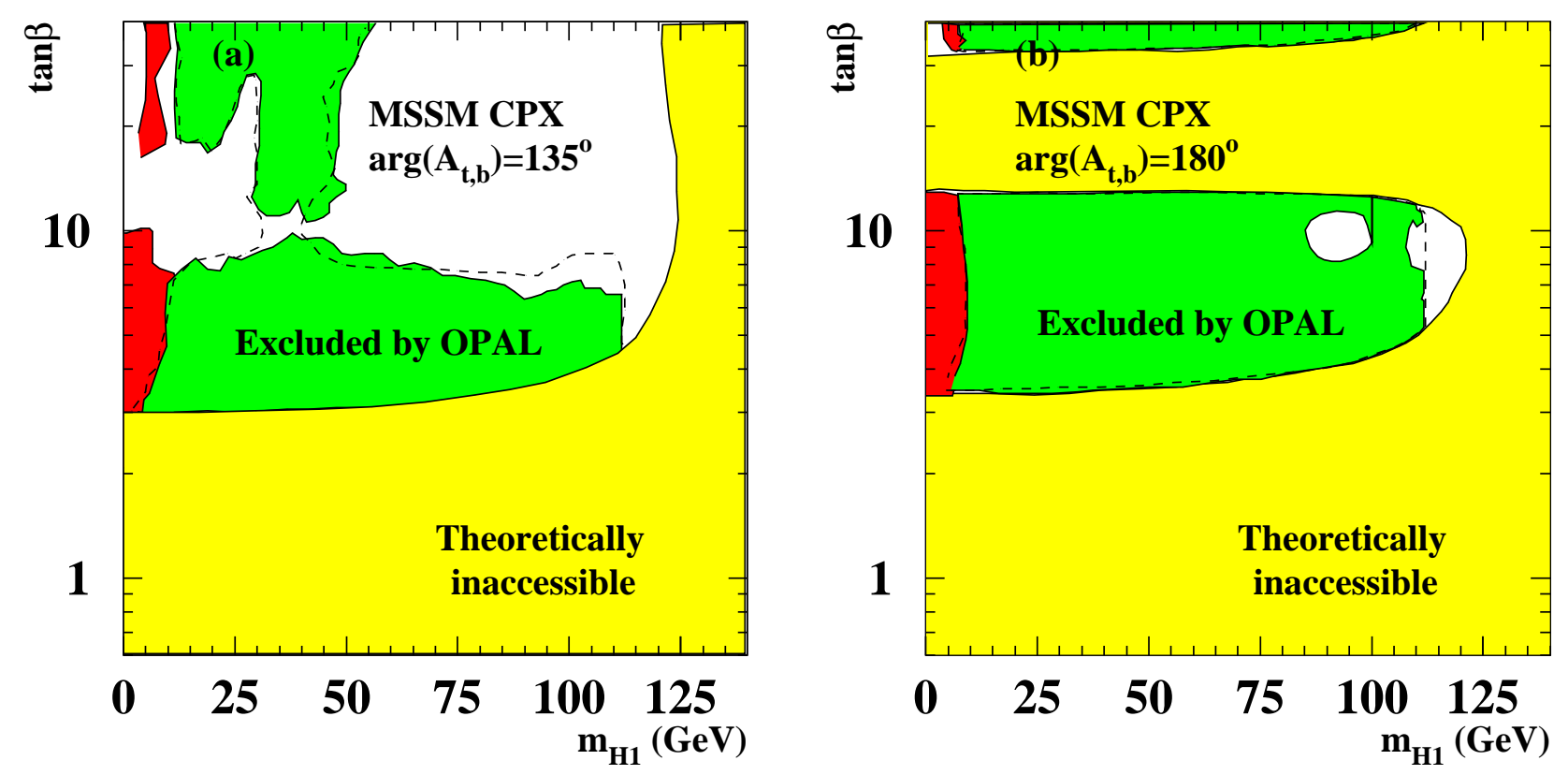

Figure 26: The CPX MSSM 95\% CL exclusion areas in the $\left(m_{\mathrm{H}_{1}}, \tan \beta\right)$ plane, using scans with (a) $\arg \left(A_{\mathrm{t}, \mathrm{b}}\right)=\arg \left(m_{\tilde{\mathrm{g}}}\right)=135^{\circ}$ and $(\mathrm{b}) \arg \left(A_{\mathrm{t}, \mathrm{b}}\right)=\arg \left(m_{\tilde{\mathrm{g}}}\right)=180^{\circ}$. See Fig. 14 for the notation. 

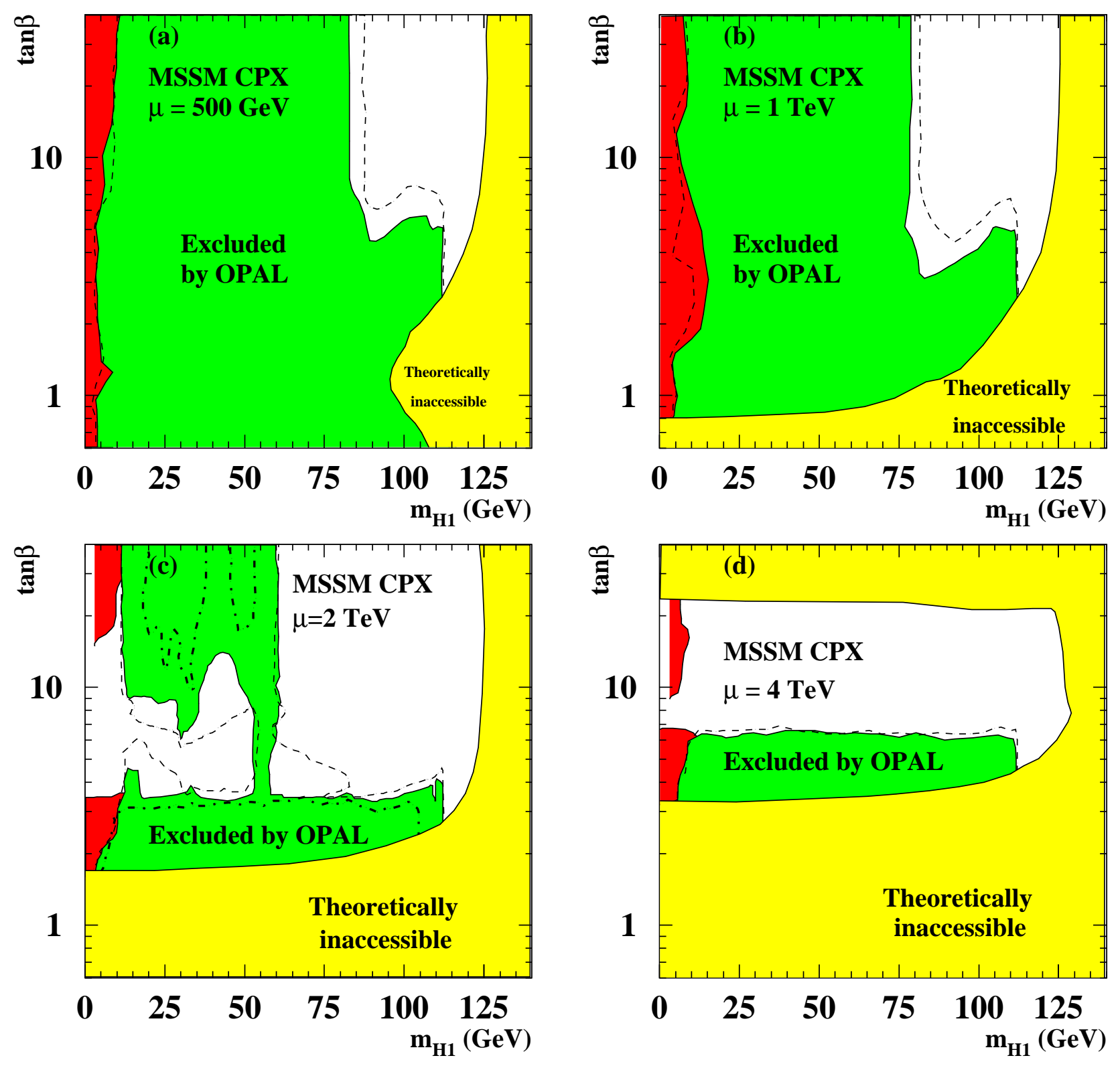

Figure 27: The CPX MSSM 95\% CL exclusion areas in the $\left(m_{\mathrm{H}_{1}}, \tan \beta\right)$ plane, using scans with (a) $\mu=500 \mathrm{GeV}$, (b) $\mu=1000 \mathrm{GeV}$, (c) $\mu=2000 \mathrm{GeV}$ (CPX) and (d) $\mu=4000 \mathrm{GeV}$. See Fig. 14 for the notation. 

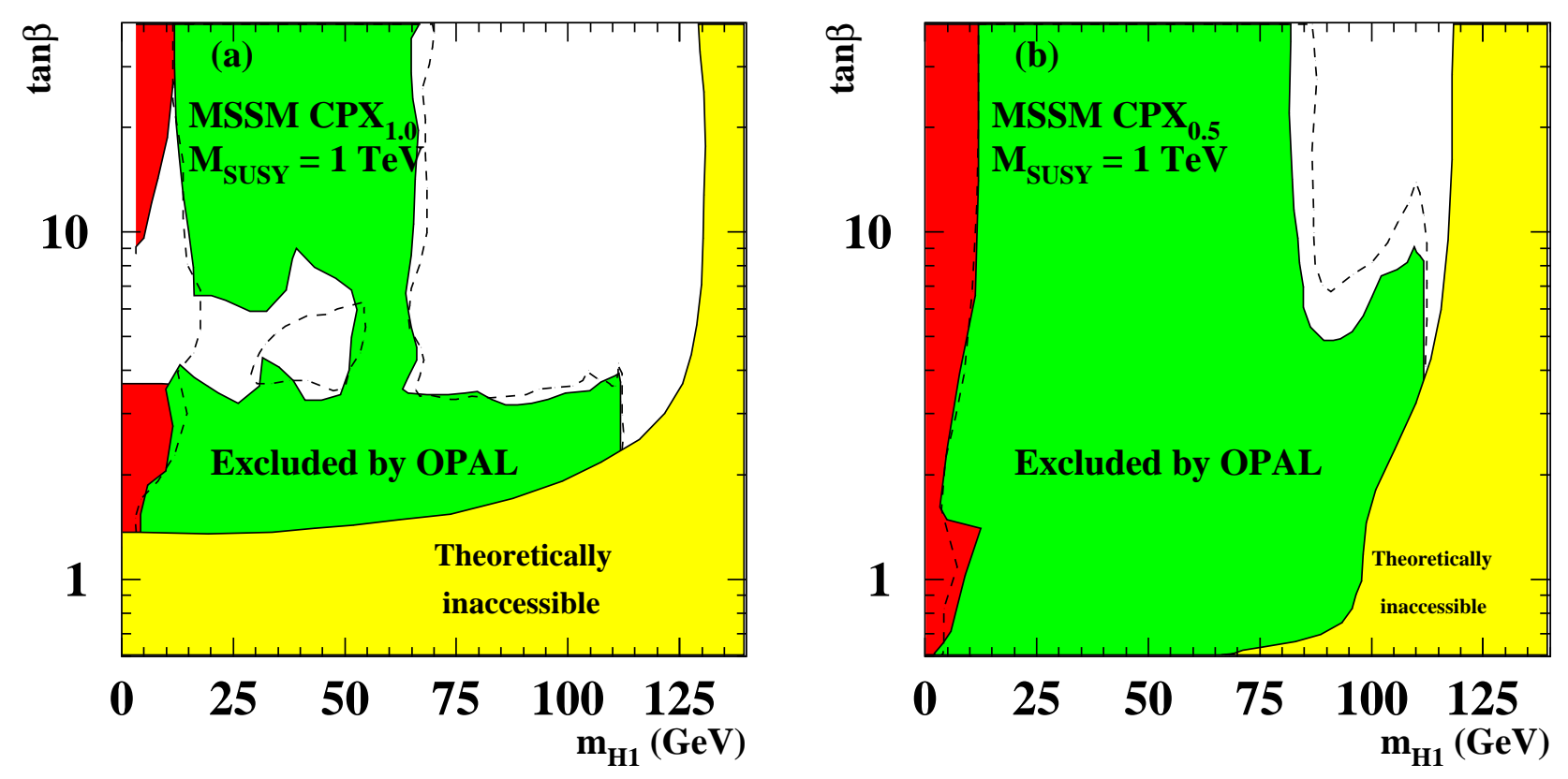

Figure 28: The CPX MSSM 95\% CL exclusion areas in the $\left(m_{\mathrm{H}_{1}}, \tan \beta\right)$ plane, using scans (a) preserving the $C P X$ ratios of $\mu, A_{\mathrm{b}, \mathrm{t}}$ and $m_{\mathrm{SUSY}}$, using $m_{\mathrm{SUSY}}=1 \mathrm{TeV}$, and (b) $m_{\mathrm{SUSY}}=1 \mathrm{TeV}$ keeping $\mu$ and $A_{\mathrm{b}, \mathrm{t}}$ at their CPX values. See Fig. 14 for the notation. 